\title{
Finite Element Analysis of Bone
}

\section{Remodelling Around an Uncemented \\ Resurfaced Femoral Head}

by

\author{
Timothy Elgin
}

B.Eng. in Mechanical Engineering, Carleton University, 2008

A thesis submitted to the Faculty of Graduate Studies and Research in partial fulfillment of the requirements for the degree of

Master of Applied Science

in

Mechanical Engineering

Carleton University,

Ottawa, Ontario

(C) 2010, Timothy Elgin 


$\begin{array}{ll}\begin{array}{l}\text { Library and Archives } \\ \text { Canada }\end{array} & \begin{array}{l}\text { Bibliotheque et } \\ \text { Archives Canada }\end{array} \\ \begin{array}{l}\text { Published Heritage } \\ \text { Branch }\end{array} & \begin{array}{l}\text { Direction du } \\ \text { Patrimoine de l'édition }\end{array} \\ \begin{array}{l}\text { 395 Wellington Street } \\ \text { Ottawa ON K1A ON4 } \\ \text { Canada }\end{array} & \begin{array}{l}\text { 395, rue Wellington } \\ \text { Ottawa ON K1A ON4 } \\ \text { Canada }\end{array}\end{array}$

Your file Votre référence

ISBN: 978-0-494-71523-9

Our file Notre référence

ISBN: 978-0-494-71523-9

NOTICE:

The author has granted a nonexclusive license allowing Library and Archives Canada to reproduce, publish, archive, preserve, conserve, communicate to the public by telecommunication or on the Internet, loan, distribute and sell theses worldwide, for commercial or noncommercial purposes, in microform, paper, electronic and/or any other formats.

The author retains copyright ownership and moral rights in this thesis. Neither the thesis nor substantial extracts from it may be printed or otherwise reproduced without the author's permission.
AVIS:

L'auteur a accordé une licence non exclusive permettant à la Bibliothèque et Archives Canada de reproduire, publier, archiver, sauvegarder, conserver, transmettre au public par télécommunication ou par l'Internet, prêter, distribuer et vendre des thèses partout dans le monde, à des fins commerciales ou autres, sur support microforme, papier, électronique et/ou autres formats.

L'auteur conserve la propriété du droit d'auteur et des droits moraux qui protège cette thèse. $\mathrm{Ni}$ la thèse ni des extraits substantiels de celle-ci ne doivent être imprimés ou autrement reproduits sans son autorisation.
In compliance with the Canadian Privacy Act some supporting forms may have been removed from this thesis.

While these forms may be included in the document page count, their removal does not represent any loss of content from the thesis.
Conformément à la loi canadienne sur la protection de la vie privée, quelques formulaires secondaires ont été enlevés de cette thèse.

Bien que ces formulaires aient inclus dans la pagination, il n'y aura aucun contenu manquant.

\section{Canadä}




\section{Abstract}

Hip resurfacing is a conservative hip joint replacement surgery that, in contrast to a traditional total hip replacement preserves the bone stock in the proximal femur. Traditionally, the femoral component of the hip resurfacing implant is fixed to the bone with a PMMA bone cement. More recently, however, cementless resurfacing designs have been undergoing clinical trials to alleviate the disadvantages of bone cement. Many early design iterations of these implants fail on account of femoral neck narrowing - a suspected consequence of stress shielding in the femoral head and neck. This study investigates the biomechanical cause of this failures with an experimentally validated adaptive finite element model of a proximal human femur. The model was fitted with a commercially available cementless resurfacing implant in order to investigate the effects of stress shielding in the femur through simulation of the bone remodelling in key regions. Three separate clinically relevant cases are investigated: complete fixation of the implant and peg, friction based contact across the implant surface and the peg, and a hybrid arrangement where the implant surface is fixed but the peg experiences frictional contact. Cases in which the inner surface of the implant is fixed to the bony surface demonstrate severe resorption of the bone tissue in the proximal femoral head; particularly in the regions above the implant peg. Fixing the peg has a positive impact in the distal region below the peg, but increases the degree of stress shielding in the upper aspect of the femoral neck and other regions away from the stem. The three tests all show significant narrowing of the cortex above the implant stem. Changing the contact properties has a large influence on the degree and nature of the stress shielding in the femoral head and neck. For the implant design, this translates to careful selection of regions that are to be coated to promote biological fixation through bone ingrowth. 


\section{Acknowledgements}

Foremost, I would like to thank my thesis supervisor, Prof. H. Frei. Without his enthusiasm towards biomechanics and orthopaedic research I may not have taken the first step and experienced the field for myself. His dedication as a supervisor and mentor has provided me with opportunities for learning and advancement for which I will be forever thankful. I would also like to thank Andrew Speirs, as both a mentor and fellow student. It was with his input, insight, and assistance that I was able to succeed in my studies.

In addition, I wish to express my appreciation towards all the staff of the Mechanical and Aerospace Engineering departmental office at Carleton University. Their patience with my "skills" in paperwork and administration was seemingly endless. Finally, I must thank the research institute and hospital staff members who were involved in this work, even in the slightest, for helping me make the most of this thesis. 


\section{Table of Contents}

\section{Chapter 1}

$\begin{array}{ll}\text { Introduction and Background } & 12\end{array}$

1.1 - Introduction 12

$\begin{array}{ll}1.2 & \text { - Basic Biomechanics } \\ & 13\end{array}$

1.2.1 - Reference Terminology 14

$\begin{array}{ll}1.2 .2 \text { - Bone } & 15\end{array}$

1.2.2.1 - Composition and Structure 16

1.2.2.2 - Structural Properties 20

1.2.2.3 - Osteoporosis 22

1.2.2.4 - Bone Remodelling 24

1.2.3 - Hip Joint Anatomy and Biomechanics 27

1.2.3.1 - Motion and Dynamics 29

1.2.3.2 - Relevant Hip Injuries and Diseases 31

1.3 - Hip Resurfacing Arthroplasty 33

1.3.1 - Current HRA Designs $\quad 35$

1.4 - FEA in Bone Remodelling and Arthroplasty 39

1.4.1 - Computational Model for Bone Adaptation 40

\section{Chapter 2}

Model Development and Validation $\quad 44$

2.1 - Constructing the 3D Femur Model 46

2.1.1 - Image Thresholding and Obtaining Point Cloud Data $\quad 47$

2.1.2 - Surface Wrapping and Solidification 49 
2.2 - Designing and Fitting the Implant Model

2.2.1 - Femoral Resurfacing Implant Model

2.2.2 - Alignment of the Implant Model

2.3 - Assembly and Meshing

58

2.3.1 - Boolean Operations

58

2.3.2 - Mesh Design

60

2.3.2.1 - Element Type: C3D10M

62

2.3.2.2 - Mesh Summary

62

2.4 - Assigning Material Information

63

2.4.1 - Calibration of CT Intensities 64

2.4.1.1 - The Calibration Phantom 64

2.4.1.2 - Density Estimation 65

2.4.3 - Assigning Element Properties 70

2.5 - Fortran Subroutines

2.5.1 - Baseline Analysis Subroutine 73

2.5.2 - The Remodelling Subroutine 75

2.5.3 - Subroutine Validation 77

2.6 - Model Validation

79

2.6.1 - Mechanical Test 79

2.6.2 - Abaqus Analysis 82

2.6.3 - Comparison 83

2.7 - Implementation 88

2.7.1 - Baseline Analysis 89

2.7.2 - Remodelling Analysis 91 


\section{Chapter 3}

$\begin{array}{ll}\text { Results } & 96\end{array}$

3.1 - The Implant's Effect on the Stimulus Field 96

3.2 - Convergence of the Remodelling Routine 99

3.3 - Final Density in the Resurfaced Femoral Head 100

Chapter 4

$\begin{array}{ll}\text { Discussion } & 105\end{array}$

4.1 - Performance of the Remodelling Analysis 105

4.1.1 - Mesh and Material Properties 106

$\begin{array}{ll}4.1 .2 \text { - Contact Properties } & 107\end{array}$

$\begin{array}{ll}4.1 .3 \text { - Initial Reference Stimulus } & 108\end{array}$

4.2 - Bone Remodelling After Uncemented Resurfacing 108

4.2.1 - Resorption Under the Implant Cap 109

4.2.2 - The Effect of the Metaphyseal Peg 111

4.2.3 - Implant Redesigning Based on Observations 112

4.3 - Comparison to Similar Studies $\quad 115$

$\begin{array}{ll}\text { 4.3.1 - Recent Publications and Results } & 115\end{array}$

$\begin{array}{ll}\text { 4.3.2 - Qualitative and Quantitative Contrast } & 117\end{array}$

Chapter 5

$\begin{array}{ll}\text { Conclusions } & 119\end{array}$

$\begin{array}{lr}5.1-\text { Contributions } & 121\end{array}$

$\begin{array}{lr}\text { References } & 122\end{array}$

Appendix A: Matlab Routine $\quad 134$ 
Appendix B: Fortran Subroutines

B1: Baseline Routine $\quad 135$

$\begin{array}{ll}\text { B2: Remodelling Routine } & 137\end{array}$

Appendix C: Remodelling Validation Results $\quad 140$

Appendix D: Equipment and Apparatus 143

Appendix E: Data from Mechanical Testing 144

Appendix F: Density Variation During Analyses $\quad 145$

Appendix G: Final Changes in Density 148

Appendix H: Quilt Plot for Final Density Changes 152 


\section{List of Figures}

1.1 Three planes of the human body 14

$\begin{array}{lll}1.2 & \text { Microscopic structure of bone tissue } & 18\end{array}$

1.3 Trabecular bone within the proximal femoral head 19

1.4 Stress-strain behaviour of cortical and trabecular bone 21

$\begin{array}{lll}1.5 & \text { Fracture fixation plate } & 26\end{array}$

1.6 Simplification of the human hip joint 28

$\begin{array}{lll}1.7 & \text { Contact forces in the hip } & 31\end{array}$

1.8 Side by side comparison between hip resurfacing arthroplasty 33 and conventional hip replacement

1.9 Principle components of a hip resurfacing implant 36

$\begin{array}{lll}1.10 & \text { Examples of modern hip resurfacing implants } & 37\end{array}$

$\begin{array}{lll}1.11 & \text { Femoral neck narrowing } & 38\end{array}$

1.12 Visual representation of the bone remodelling algorithm 41

2.1 Flowchart demonstrating the processes involved in 45 developing an adaptive bone model for the resurfaced proximal femur

2.2 OsiriX point cloud data contrasting the resulting isometric $\quad 50$ models limited to $500 \mathrm{HU}$ and $300 \mathrm{HU}$

2.3 Cleaned facetted femur as represented in Pro/ENGINEER 51

2.4 Preparation process for the closed surface model of the femur $\quad 54$

$\begin{array}{lll}2.5 & \text { Implant model design } & 56\end{array}$

2.6 Alignment of the implant model on the intact femur $\quad 57$

$\begin{array}{lll}2.7 & \text { Three principle modelling phases } & 59\end{array}$

2.8 Patran tetrahedral mesh of the femur models 61 
$\begin{array}{lll}2.9 & \text { CT calibration phantom } & 65\end{array}$

$2.10 \quad$ Sampling ROI intensities in OsiriX 68

2.11 Visualization of CT and mesh data in Amira 71

$2.12 \quad$ Specimen loading schematic $\quad 81$

2.13 Cadaveric femur outfitted with strain gauge rosettes 80

2.14 Density field distribution within the intact femur model 84

$\begin{array}{lll}2.15 & \text { Three implant interface models } & 89\end{array}$

$\begin{array}{lll}2.16 & \text { Femur mode loading and boundary conditions } & 90\end{array}$

$\begin{array}{lll}2.17 & \text { Surfaces used for implant-femur interaction } & 93\end{array}$

3.1 Enumerated regions of the resurfaced femoral model $\quad 97$

$\begin{array}{lll}3.2 & \text { Initial remodelling stimulus for each implant scenario } & 98\end{array}$

3.3 Final remodelling stimulus for each implant scenario $\quad 100$

3.4 Final bone density distribution for each implant scenario $\quad 101$

3.5 Absolute change in bone density for each implant scenario 104

4.1 Surface contact area for selectively fixed model $\quad 113$

4.2 Final change in bone density under selectively tied implant 114

C1 Baseline stimulus distribution in cantilever beam $\quad 140$

C2 Stimulus distribution on step 2 of remodelling $\quad 140$

C3 Stimulus distribution on step 4 of remodelling 141

C4 Stimulus distribution on step 10 of remodelling 141

C5 Final density distribution after 10th step of remodelling $\quad 142$

F1 Bone density during analysis of the fully fixed model 146

F2 Bone density during analysis of the fully friction model 146

F3 Bone density during analysis of the fixed-friction model $\quad 147$

G1 Absolute change in bone density under fully fixed implant $\quad 148$ 
G2 Absolute change in bone density under fully friction implant

G3 Absolute change in bone density under fixed-friction implant

G4 Absolute change in bone density under selectively fixed implant

H1 Absolute change in bone density for each implant scenario output as a quilt 


\section{List of Tables}

$1.1 \quad$ Approximate composition of the extracellular matrix of bone $\quad 16$

$1.2 \quad$ Approximate mechanical attributes of cortical and trabecular 23

bone relative to biomedical engineering materials

$2.1 \quad$ Mesh information for resurfaced and intact femur models $\quad 63$

2.2 Phantom reference material composition 64

$\begin{array}{lll}2.3 & \text { Reference rod intensities } & 67\end{array}$

2.4 Data array used for linear regression of Equation $2.5 \quad 69$

$\begin{array}{lll}2.5 & \text { List of all state and field variables used in the remodelling } & 74\end{array}$

subroutine

2.6 Strain reading comparison between cadaveric femur and $\quad 85$

FEM results

D1 Strain data gathered from the cadaveric femur $\quad 144$

D2 Displacement data gathered from the cadaveric femur $\quad 144$ 


\section{Chapter 1}

\section{Introduction and Background}

\section{1 - Introduction}

The goal of this thesis was to develop an adaptive finite element model to investigate the effects of stress shielding and bone remodelling around a cementless metallic hip resurfacing implant. Metal-on-metal hip resurfacing is a relatively recent surgical procedure designed primarily to treat younger patients suffering from different forms of arthritis or other illness or injury that may effect the performance of the hip joint. Hip resurfacing arthroplasty is sometimes selected over total hip arthroplasty (the more conventional form of hip replacement surgery) on account of its conservative nature and promising outlook in younger patients.

Most implant designs follow an almost identical template and few manufacturers have varied from the accepted approach. There is, however, a much ongoing debate regarding the design of modern resurfacing implants, in particular, on the omission of bone cement on the femoral side implant. Some orthopaedic device companies have begun offering cementless implants that are coated in a manner that promotes in-growth of bone tissue onto the implant surface in an effort to provide better bone integration and avoid the negative effects of bone cement. The thermal setting process of bone cements can damage surrounding bone tissue, and although the cement layer provides a decent 
mechanical boundary between the implant and the bone, it prevents long term integration of bone tissue around the implant.

Early failures of the femoral component of hip resurfacing implants through loosening or femoral neck fracture are often attributed to bone resorption within the femoral head (a decrease in bone density attributed to stress shielding) and femoral neck narrowing. These types of failure have been observed in recent clinical trials of both cemented and cementless resurfacing implants, but are not fully understood [1 - 3]. Ultimately, the model developed in this study is used as a design tool to aid in predicting the outcome of changes to current cementless hip resurfacing implants and modelling stress shielding and bone resorption around the femoral resurfacing implant. Some background in bone biology, hip biomechanics, and hip resurfacing arthroplasty is perhaps in order.

\section{2 - Basic Biomechanics}

This thesis is concerned with the biomechanical evaluation of a human femur implanted with a hip resurfacing implant. Although design and analysis was performed from an engineering standpoint, a basic knowledge of human anatomy and physiology is also necessary. Therefore, this chapter summarizes the basic anatomy, physiology, biology, and biomechanics relevant to the hip joint. 


\subsection{1 - Reference Terminology}

The human body is assigned a $\mathbb{B}$-dimensional planar coordinate system to provide a unified means of directional referencing. The planes are defined relative to an upright figure with arms down and palms facing forward (as in Figure 1.1). This is the international standard body position. A plane formed by cutting the figure from left to right, down the length of its spine, is called the coronal (frontal) plane. A cutting plane placed 90 degrees to the coronal plane, but still

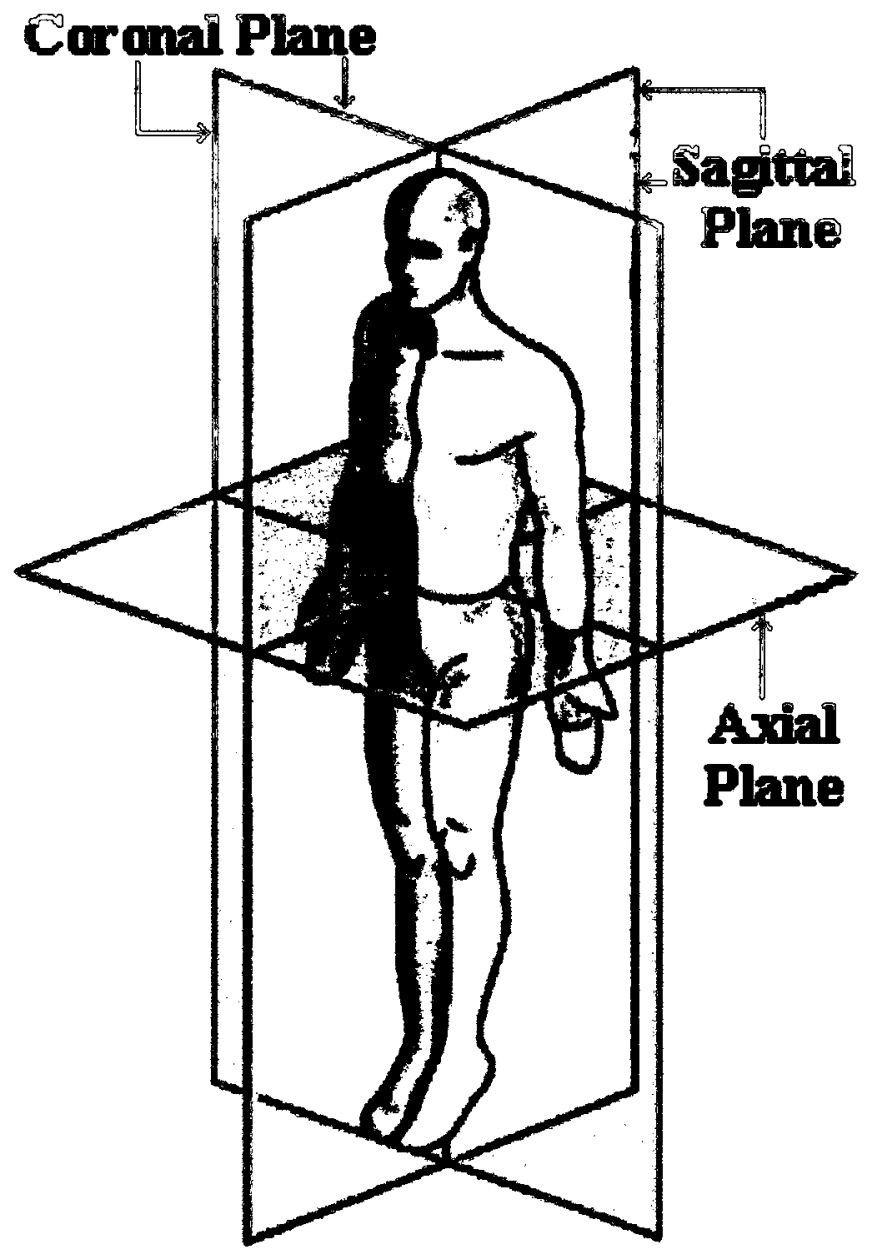

Figure 1.1: Three planes of the human body [4]. 
parallel to the spine, forms the sagittal (median) plane. Finally, perpendicular to both sagittal and coronal planes lies the transverse (axial) plane.

In addition to the specific coordinate system, other frame-of-reference terminology exists to clarify directions and orientations in situations where there is often no clear definition of "up" or "down". When working in a coronal plane, regions toward the centerline of the body are identified as medial. Those towards the outside of the body (left or right) are said to be lateral. Similarly, the terms "up and down" are replaced by superior and inferior respectively. When referring to directions in a sagittal plane, "front and back" are replaced by anterior and posterior. Finally, the terms proximal and distal are used to describe areas of interest that are close to or away from the torso.

\subsection{2 - Bone}

Bone is a connective tissue that plays a variety of roles in human anatomy [5]. It is one of the hardest compounds found within the body and many, though not all, of its roles are related to the protection of vital organs and to structural support and motion. Because an extensive vascular system provides all regions of the bone tissue with an ample supply of blood, bone has a great capacity for selfrepair and is capable of altering its structural and material properties based on changes in mechanical loading [5]. This property, often termed bone remodelling, plays a vital role during injury recovery and during periods of increased or 
decreased use. It is therefore of particular interest to this study. A more extensive discussion of bone remodelling is included in Section 1.2.2.4.

\subsubsection{1 - Composition and Structure}

Similar to other connective tissues, bone consists of cells surrounded by an extracellular matrix produced by those cells. A large percentage of its dry weight is accounted for by inorganic matrix matter - primarily calcium and phosphate. This predominantly mineral matrix is what provides bone with its hardness and compressive strength. Bone matter also serves as a storage system for many of the mineral dependencies of the body (such as calcium). Table 1.1 outlines the basic composition of the extracellular matrix of bone. The exact composition of bone differs depending on anatomical location, age, and medical history. There are also a number of diseases and deficiencies that affect the quality and composition of bone [6].

\begin{tabular}{|l|c|}
\hline Component & Mass (\%) \\
\hline Mineral phase & 70 \\
\hline Organic Matrix & \\
\hline Collagen & 18 \\
\hline Non-collagenous proteins and proteoglycans & 2 \\
\hline Water & 10 \\
\hline
\end{tabular}

Table 1.1: Approximate composition of the extracellular matrix of bone. [Y] 
The mineral phase of bone is held together by organic elements of the extracellular matrix, primarily collagen. Collagen is a protein structure that plays an important role in all mammalian connective tissue. It is a tough fiberlike structure that is highly resistant to tensile deformation, though otherwise pliable. By volume, collagen makes up the majority of the extracellular material [5] though it only claims $18-20 \%$ by mass. The rest of the matrix ( $2 \%$ by mass) is an organic protein gel that acts both as a ground substance for the mineral and collagen phases and a binding substance. Water also has a considerable presence $(\sim 10 \%$ by mass $)$ in bone tissue.

The microscopic organization of bone is governed primarily by its principal structural building block, the osteon. Osteons are microscopic, cannulated, cylindrical arrangements of bone tissue (Figure 1.2). Each osteon is formed from concentric layers of bone tissue surrounding an axial canal, known as a Haversian canal. The longitudinal canals are joined by a transverse group of canals (Volkmann's canals). The system of canals contains the blood vessels responsible for nourishing the bone tissue and cells, which are situated between the concentric layers of mineralized bone tissue. Each individual osteon is surrounded by a layer of protein based ground substance that forms the "cement line". The bone tissue within the osteon contains a high concentration of collagen fibers that provides good mechanical strength; however, the cement layer lacks this collagen concentration. Consequently, these boundaries are often the weakest point in microscopic bone [5]. The osteons are usually arranged 


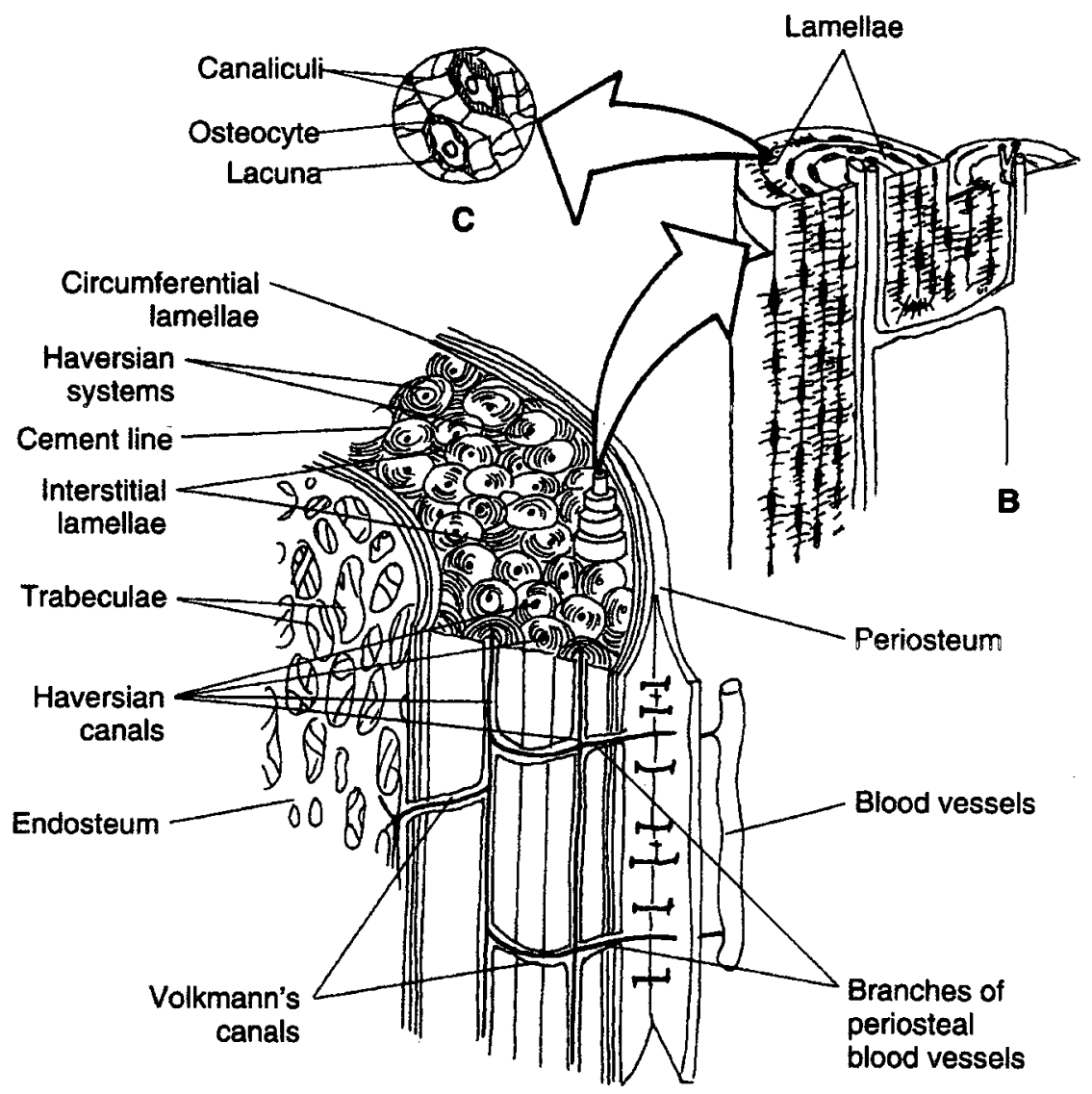

Figure 1.2: Microscopic structure of bone tissue [5].

uniformly but offset longitudinally and they may branch into different directions. The orientation of osteons plays a role in the mechanical strength and failure limits of bones under different loading directions.

The macroscopic structure of the bone also has a significant influence on its material properties and it varies greatly throughout the body and within individual bones. Bone structure can be classified as either cortical bone or cancellous (trabecular) bone - which differ greatly in macrostructure and, consequently, mechanical behaviour. Cortical bone forms the hard shell of most 
bones. It has a relatively high density, toughness, and elastic modulus. Within the cortical shell (cortex) lies a porous matrix of trabecular bone, arranged for optimal distribution of load (Figure 1.3). Although the microstructure of trabecular bone is similar to that of cortical, at a higher level the bone is much less dense and resembles a sponge or mesh. The porosity of trabecular bone can vary greatly within a single bone, and is known to develop in a load-dependent manner. For example, the distribution of the thin trabecular plates that make up the bony structure in the head of the femur (the long bone of the upper leg) is related to the anatomical loads the bone is subjected to [8].

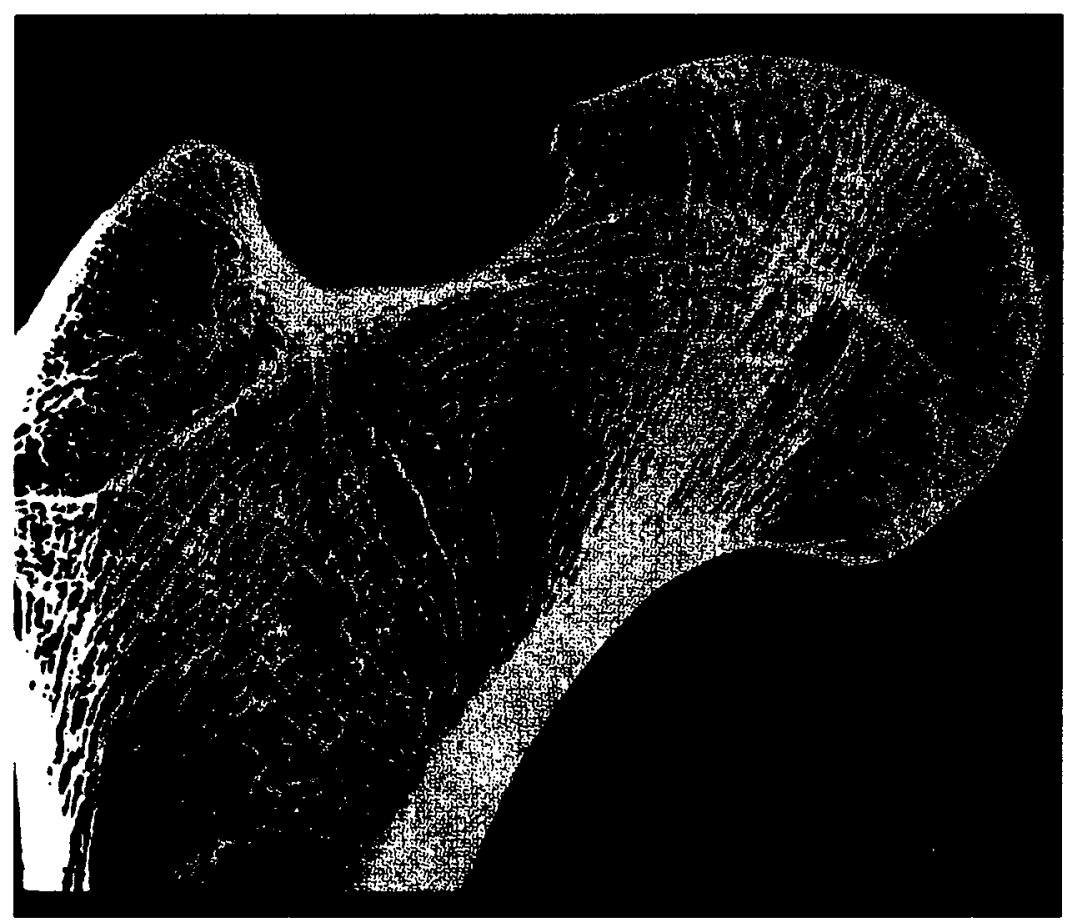

Figure 1.3: Trabecular bone within the proximal femoral head. The head is highly porous, with trabecular bone distributed along lines of elevated strain [9]. 


\subsubsection{2 - Structural Properties}

Bone has been compared to a two-phase composite material $[10,11]$. The strong but brittle calcium phosphate mineral phase is reinforced with weaker yet far tougher collagen fibers. Bone demonstrates anisotropic properties however it is often modelled as an orthotropic or isotropic elastic material [12].

The strength and elastic modulus of cortical and trabecular bone differ greatly. In addition, the properties of bone also vary depending on age, health, gender, and loading history [5]. Figure 1.4 demonstrates the approximate relationship between apparent bone density (the mass of bone present in an enclosed volume that may include vacancies and non-bony tissue) and elastoplastic properties. Stemming from the work performed by Carter and Hayes in 1977 [10], a number of studies have proposed relationships that define its elastic modulus, E, relative to its apparently density $[13-15]$. The popular model introduced by Carter and Hayes [10], often used with finite element analysis, proposes that the elastic modulus is modelled as a power function of both strain rate and density, $\rho$, as shown in Equation 1.1.

$$
E=3790 \cdot \dot{\varepsilon}^{0.06} \cdot \rho^{3}
$$

Weinans et. al [16] suggested the strain rate term can be assumed equal to 1 (making a rate independent relationship) because of the negligible effect it has 


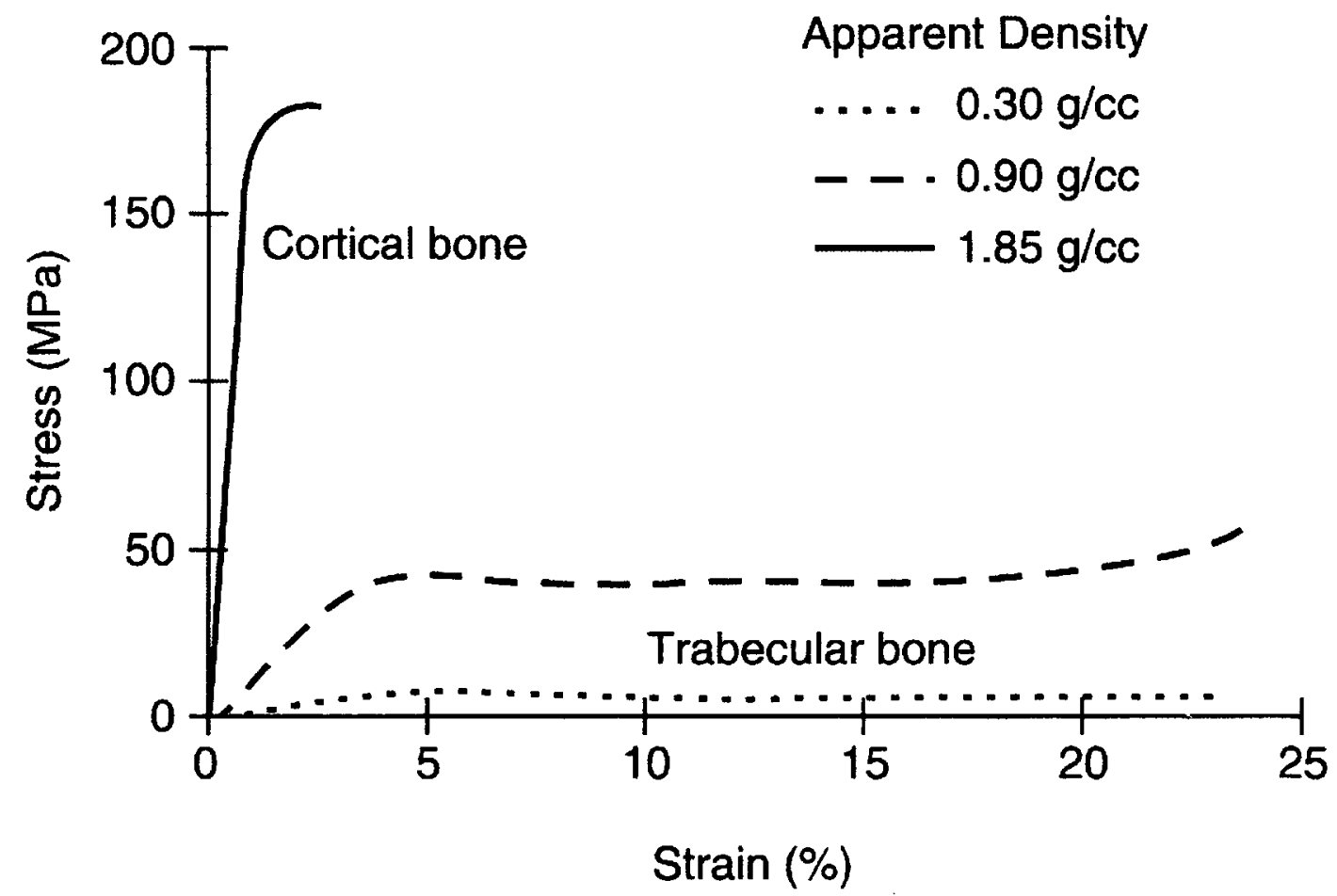

Figure 1.4: Stress-strain behaviour of cortical and trabecular bone [5].

been shown to have in most models. A similar relationship was applied to a cemented hip resurfacing bone remodelling problem in 2006 by Gupta [15]. Equation 1.2 is inherently strain rate independent and has a smaller exponent on the density term.

$$
E=7281 \cdot \rho^{1.52}
$$

Eq. 1.2

Considering either one of these relationships, it is clear that trabecular bone has a significantly lower elastic modulus than cortical bone. This is 
attributed to its porous nature and therefore its low apparent bone density. It is important to note that while cortical bone has a failure strain of approximately $2.0 \%$, trabecular bone is known to have excellent compressive strain limits; however, it still exhibits brittle failure tendencies [5]. Keaveny et. al. [17] demonstrated that the porous nature of trabecular bone gives it a larger capacity for energy storage than cortical bone.

Although many sources list the approximate mechanical properties of bone, either by bone or type of bone tissue, the only value such tables provide is a general concept of their properties relative to those of typical engineering materials and of other mammalian bones. Because the actual properties of a given bone (or bone sample) are unique to that sample, its attributes should be determined experimentally rather than estimated using values published in the literature. Table 1.2 provides the elastic modulus and ultimate strength of bone relative to common metals used in the biomedical field. Values listed for both cortical and trabecular bone vary greatly between sources.

\subsubsection{3 - Osteoporosis}

Osteoporosis is a bone condition that indicates a drastic decrease of bone density and structural integrity and therefore an increased susceptibility to bone fracture and failure [18]. Osteoporosis is classified as a bone mineral density (BMD) that is 2.5 or more standard deviations from the mean value for healthy

young adults. A less severe form of osteoporosis, osteopenia, is defined as a BMD 


\begin{tabular}{|l|c|c|c|}
\hline & $\begin{array}{c}\text { Ultimate Strength } \\
(\mathbf{M P a})\end{array}$ & $\begin{array}{c}\text { Modulus } \\
(\mathbf{G P a})\end{array}$ & Percent Elongation \\
\hline Co-Cr alloys & & & 8 \\
\hline Cast & 600 & 220 & 15 \\
\hline Forged & 950 & 210 & 10 \\
\hline Stainless Steel & 850 & 110 & 15 \\
\hline Titanium & 900 & 2.0 & $2-4$ \\
\hline Bone Cement & 20 & 350 & $<2$ \\
\hline Ceramic & 300 & & 3 \\
\hline Cortical Bone & & & 3 \\
\hline Longitudinal & 133 & 17.0 & 1 \\
\hline Tensile & 193 & 17.0 & $1-3$ \\
\hline Compressive & 63 & 3.3 & $1-3$ \\
\hline Shear & & & $2-5$ \\
\hline Transverse & 51 & 11.5 & 0.4 \\
\hline Tensile & 133 & 11.5 & \\
\hline Compressive & $8-50$ & & \\
\hline Trabecular Bone & & & \\
\hline
\end{tabular}

Table 1.2: Approximate mechanical attributes of cortical and trabecular bone relative to biomedical engineering materials. Adapted from [7].

between 1.0 and 2.5 standard deviations from the mean [6]. The weakened state of osteoporotic bone not only increases the risk of fracture, but has a detrimental effect on the healing process and implant fixation and stability $[19,20]$. Osteoporosis is positively related to age, and is most commonly seen in post menopausal woman and patients with a maternal history of osteoporotic related injuries (such as hip fracture). 


\subsubsection{4 - Bone Remodelling}

An important aspect of this study, and of skeletal biomechanics in general, is the adaptive nature of bone. Bone responds to a number of different internal and external stimuli, including hormone and mineral levels and the current state of mechanical loading [6]. The nature of bone to adapt to externally applied loads is known as Wolff's law - motivated by Julius Wolff's publication in 1892 that demonstrated the relationship between bone structure and mechanical loading [21]. Although metabolic and hormonal factors have an important influence on the quality of living bone in an organism, mechanical influences (stress and strain) are of particular interest to engineers and orthopaedic surgeons.

Remodelling occurs in two principal ways. In cortical bone, remodelling manifests itself as an increase or decrease in cortical thickness and/or bone diameter through resorption or deposition at the inner and outer cortical layers. In trabecular bone remodelling occurs through trabecular thinning, thickening and/or re-alignment [8]. Trabecular remodelling is often summarized as an increase in local bone, density motivated by an increase in load, or a decrease in density due to lower loads [5]; however, the actual process is far more complex. The true biological mechanism for sensing variations in the stimulus signal is not fully understood [22]. A number of different biomechanical stimuli, such as strain, stress and strain energy density have been proposed [23, 24]. Osteocytes, bone cells embedded within the laminar layers of bone, are thought to act as 
mechanotransductors. They detect changes in the reference stimulus (expected anatomical load) and signal to other bone cells, osteoclasts and osteoblasts, the need for deposition or resorption of bone $[23,25]$. The osteoclasts and osteoblasts are the cells responsible for the absorption and deposition of bone tissue, respectively.

Although the actual mechanism by which bone remodelling takes place is not fully understood, its impact on orthopaedic research and the design of orthopaedic implants is significant. The classic example of Wolff's law influencing the success of an implant is the fracture plate used to stabilize a broken long bone (Figure 1.5). Under normal anatomical conditions the load is transferred in its entirety by the bone tissue. Post fracture, the plate is inserted to hold the two fractured bone fragments in contact to facilitate healing. The plate is designed to maintain pressure between proximal and distal bone segments. It also carries a significant percentage of the applied loads. During early healing this allows for proper recovery; however, if the implant remains in situ too long, it prevents proper healing of adjacent bone tissue. Loads normally carried by the long bone are instead taken by the stiffer implant material. Ultimately, the change in load path can lead to resorption of bone adjacent to the fracture site and incomplete healing of the fracture itself. This condition, where local loads are decreased due to the presence of an orthopaedic implant, is termed "stress shielding" [26]. Stress shielding is an important design consideration for all metallic implants [27]. Another undesirable effect is an increase in bone 


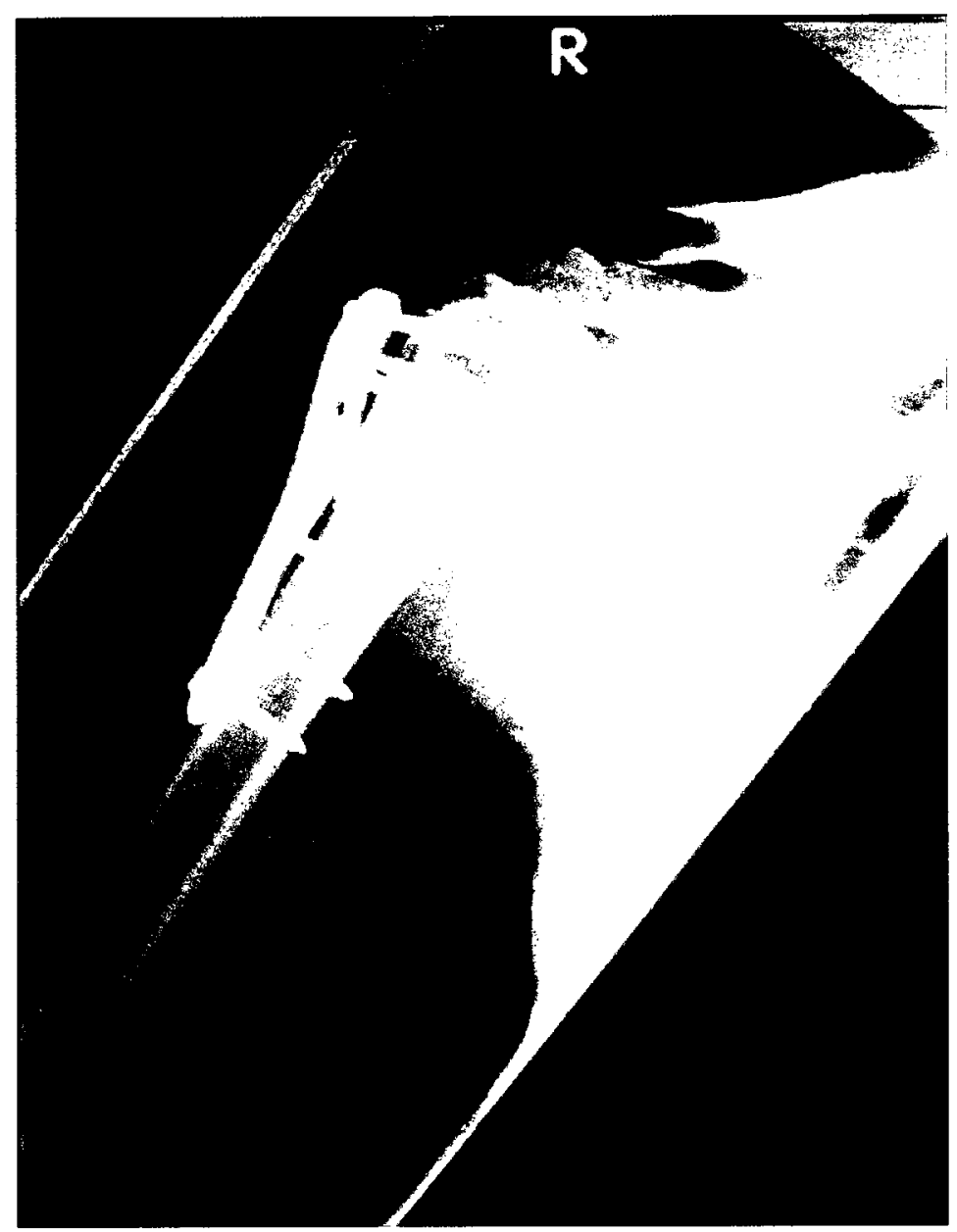

Figure 1.5: Fracture fixation plate used to repair proximal humeral fractures [28].

density around the fixation screws (hypertrophy). The screw surfaces represent a relatively small contact area but because of their stiffness, and that of the plate, the anatomical load path changes to rely more heavily on the plate and screws. Consequently, the bone surface in contact with the screws sees more load and responds through increased bone deposition [5]. The adaptive nature of bone can also be seen without the influence of implants or injury. Prolonged bed rest has been shown to result in a $1 \%$ decrease in bone mass for each week spent immobile 
[29]. Conversely, an increase in bone mass can be observed in response to strenuous exercise [30].

The adaptive nature of bone tissue adds significant challenges to the design and improvement of orthopaedic implants. Stress shielding poses a serious risk for the success of all metallic bone implants. A decrease in local bone quality may lead to aseptic implant loosening or stress concentrations at the implantbone interface. It is also possible that local stress concentrations cause hypertrophy of small contact regions thereby enhancing the effect of the stress concentrations and leading to unpredicted remodelling and poor bone-implant interface characteristics.

\subsection{3 - Hip Joint Anatomy and Biomechanics}

The human hip is an articulation joint joining the torso to the upper leg. The joint follows a ball-and-socket configuration, allows for a significant degree of motion, and is capable of carrying large loads. Because the configuration involves only two articulation surfaces, it is referred to as a "simple joint". Two anatomical bodies interact to form the hip joint: the acetabulum and the femoral head (Figure 1.6). The femoral head is roughly two-thirds of a sphere and acts as the convex aspect of the ball-and-socket joint [5]. The spherical surface is covered in articular cartilage that provides a low-friction interface and a pliable boundary layer to allow for good surface contact. Similarly, on the acetabular side (the concave aspect of the socket joint), articular cartilage provides the interaction 


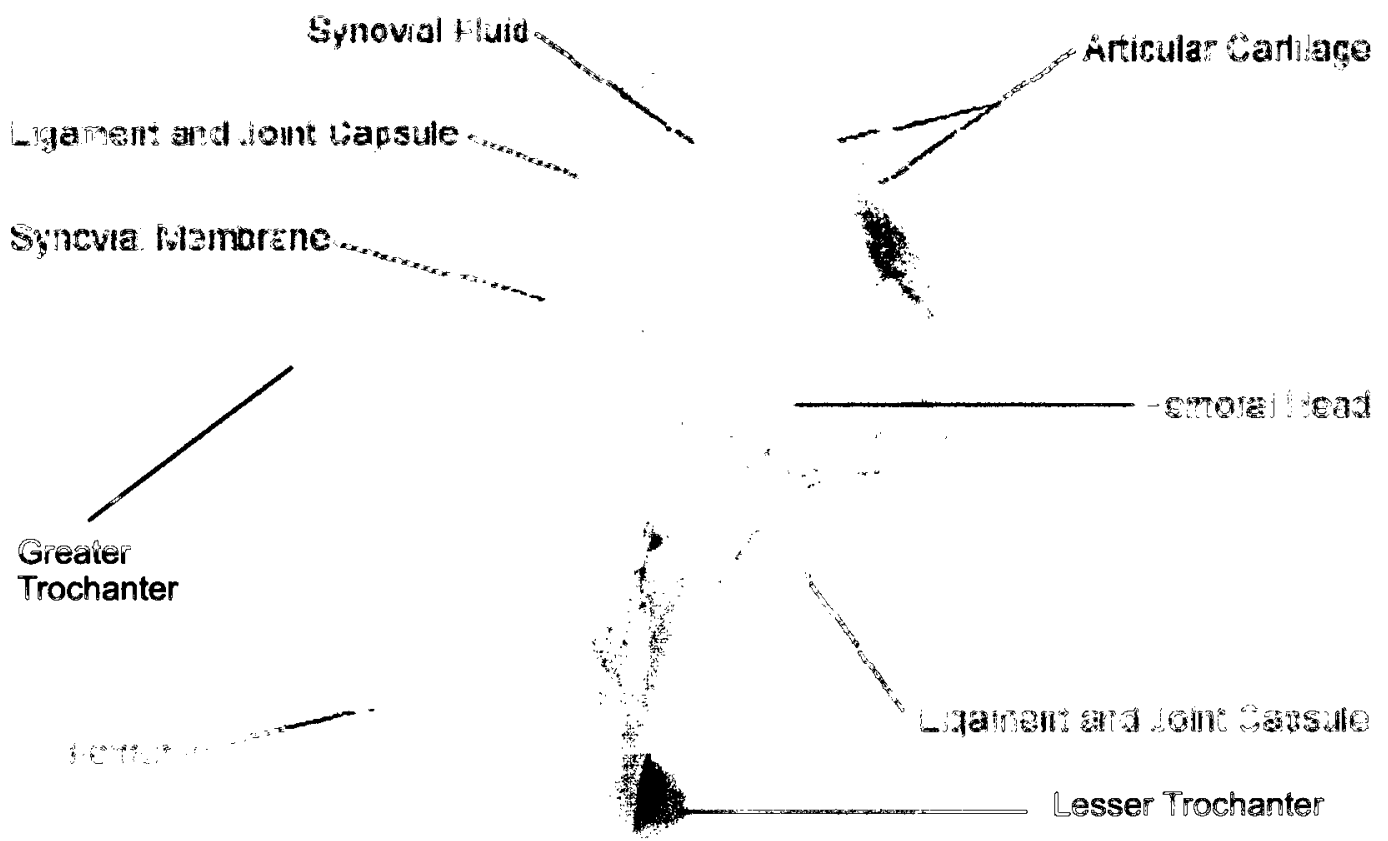

Figure 1.6: Simplification of the human hip joint. Reproduced from Columbia University Medical Center [31].

surface that thickens near the periphery of the dish. The thicker cartilage acts as a sort of collar for the femoral head that adds stability and aids in preventing dislocation. In addition, a ring of cartilage tissue surrounds the acetabulum to increase the depth of the socket. Synovial fluid provides lubrication between the cartilage layers of the acetabulum and the femoral head. The entire joint is encapsulated by three stabilizing ligaments: the iliofemoral ligament, the ischiofemoral ligament, and the pubofemoral ligament which make up the joint capsule. A fourth ligament, the ligament of the head of the femur, is attached to a fovea on the femoral head. This ligament, however, provides negligible stability, 
although it contains vasculatures responsible for providing some of the blood supply for the femoral head.

\subsubsection{1 - Motion and Dynamics}

Like other ball-and-socket joints, hip motion occurs in all three possible planes of motion: sagittal, coronal and transverse. The greatest degree of motion occurs in the sagittal plane, which is parallel to the direction of motion during normal gait. The range of motion when walking is approximately 5 degrees of extension (when the leg is swung towards the posterior) to 40 degrees flexion (anterior swing) [32]. The maximum range of motion in the sagittal plane is reported to be approximately 15 degrees of extension and 140 degrees of flexion [5]. Approximately \pm 5 degrees of internal and external rotation (motion in the axial plane) and \pm 5 degrees of adduction and abduction (side-to-side motion in the coronal plane) is also observed during normal walking [32]. Similar to motion in the sagittal plane, these values do not approach the limiting range of motion for the joint. Internal and external rotation is limited to approximately 70 degrees to 90 degrees, and adduction (medial motion) and abduction (lateral motion) are limited to 25 degrees and 30 degrees on average. Other common activities, such as squatting, ascending or descending stairs, or stooping, use a much greater portion of the hip's range of motion [33].

Forces in the intact human hip joint have been estimated using inverse kinematic observations coupled with force plate data $[34,35]$. In addition, 
Bergmann et. al. [36, 37] have performed work using hip prosthetics instrumented with force sensors that transmit the force magnitudes through use of telemetry. The work performed by Bergmann and his associates provides significant insight into the contact loads of the femoral head during normal gait and other activities, including sitting, standing, and stumbling. It was shown that during regular walking the peak compressive contact force applied through the centre of the implant head averages between 200 and $250 \%$ of body weight (BW) for most patients. The medial component reaches peak values of $50 \% \mathrm{BW}$, and the anteriorly directed force almost $25 \% \mathrm{BW}$ (Figure 1.7). In terms of force magnitude, this indicates that an $80 \mathrm{~kg}$ individual would experience hip joint loads up to $2000 \mathrm{~N}$ during normal walking due to added muscle forces required to provide balance while performing an activity, or even while standing still.

Older studies, using electromyography coupled with force plates to measure joint and muscle forces, report values between $450 \%$ and $800 \% \mathrm{BW}$ in the hip joint $[38,39]$. More recently, van den Bogert et al. [40] performed an experiment using a 12-channel accelerometer system and inverse dynamic analysis to determine joint force loads during walking, running, and skiing. This approach yielded results closer to those seen by Bergmann. It was estimated that the hip joint experiences forces between $220 \%$ and $280 \%$ body weight during walking [40]. 


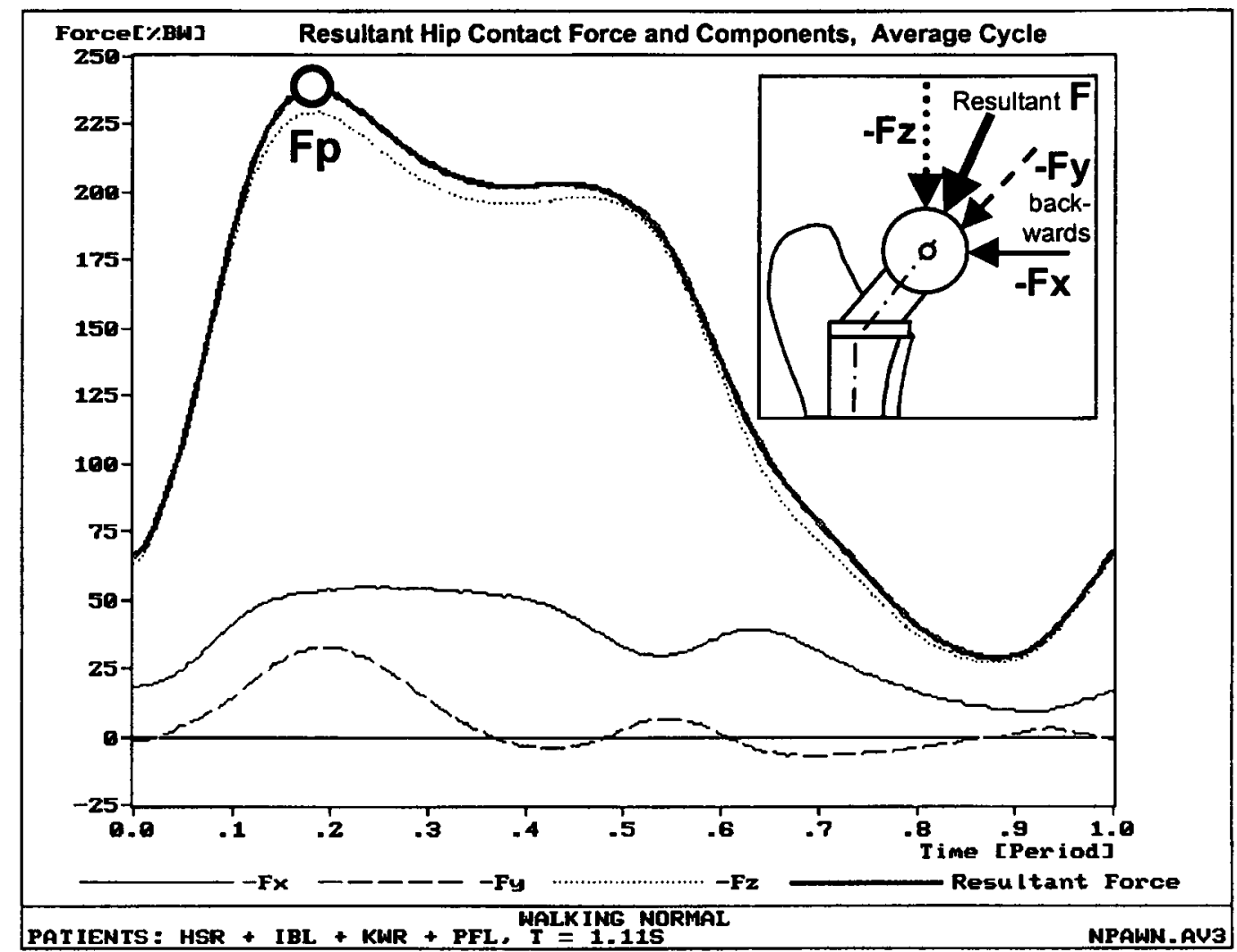

Figure 1.7: Contact forces in the hip. Forces are identified by component during one gait cycle of normal walking. The peak walking force occurs at point Fp. [36]

\subsubsection{2 - Relevant Hip Injuries and Diseases}

There are a number of conditions that reduce the effectiveness of the hip joint and cause pain. Most of these are a form of arthritis (osteoarthritis, traumatic, and rheumatoid). Osteoarthritis, one of the leading indications for hip arthroplasty, is a degenerative form of arthritis commonly associated with aging, although there is a significant population of younger patients suffering from this disease. Osteoarthritis is characterized by the degeneration of the cartilage layers 
on the femoral head and acetabulum. The loss of the contact surface within the joint can lead to bone deterioration, pain, and limited range of motion [6].

Traumatic arthritis and rheumatoid arthritis both demonstrate similar symptoms to osteoarthritis, but differ through the underlying cause. As the name implies, traumatic arthritis occurs when the joint has undergone serious injury. Many such instances are recoverable without surgical intervention, but in severe cases sections of articular cartilage may be torn or sheared from the bony surface. These pieces of cartilage must be removed surgically, and because of the poor healing properties of cartilage the joint will never fully recover. In addition, repeated damage can build up and eventually lead to severe arthritis of the joint. Rheumatoid arthritis is an auto-immune disorder that often affects synovial joints, such as the hip joint, throughout the body. Inflammation of the membrane that contains the synovial fluid within the joint leads to deterioration of the articular cartilage. Avascular necrosis, which is the death of cells and tissue due to the absence of adequate blood supply, is yet another contributor to hip joint degeneration. It can cause deterioration of both the cartilage tissue and the subchondral bone (bone below the cartilage). Finally, injury related fractures are not uncommon in the hip. Elderly patients may suffer fracture of the proximal femur or femoral neck after a standing fall; however, in young, healthy adults severe trauma is often required to cause fracture [6].

There are a number of non-surgical treatments for hip degeneration conditions but severe cases of arthritis and avascular necroses are often addressed 
with hip joint arthroplasty - replacement of the affected joint. This thesis focuses on hip resurfacing arthroplasty - a bone conserving form of hip arthroplasty.

\section{3 - Hip Resurfacing Arthroplasty}

Conventional (total) hip replacement implants, where the entire femoral head is removed and replaced with a femoral stem (Figure 1.8, right), have

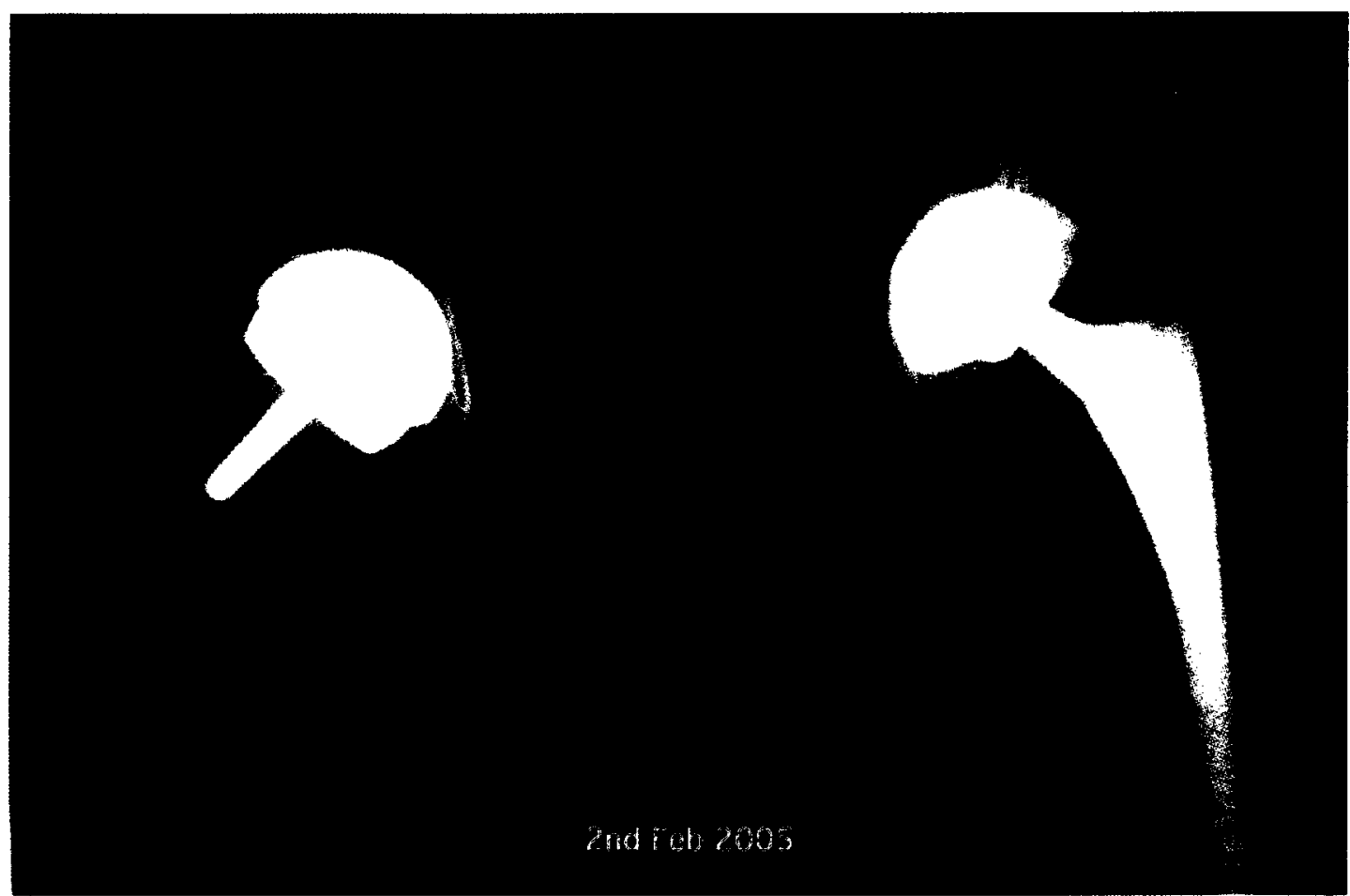

Figure 1.8: Side by side comparison of hip resurfacing arthroplasty (HRA; left side of the x-ray image) and conventional total hip replacement (right side of the $x$-ray image, left hip). The patient was originally treated with a single hip resurfacing implant in the left hip in 1996 . In 2002, the other hip was treated with HRA. Both implants were motivated by increased pain and limited joint function caused by osteoarthritis. In 2005 the original implant (1996) was revised to a total hip replacement when the patient complained of increasing pain. The cause of pain was determined to be loosening of the implant caused by tissue loss due to metallic wear particles. [41] 
demonstrated less than acceptable performance in younger and more active patients [42]. Younger patients are more inclined to maintain their pre-operation levels of physical activity after receiving a hip implant [43,44]. As a result of the apparent deficiencies of total hip arthroplasty (THA), many younger patients suffering from osteoarthritis are now being treated using hip resurfacing arthroplasty (HRA). Hip resurfacing is a conservative alternative to conventional hip replacement surgery. The modern procedure involves only the removal of the diseased layer of cartilage tissue on both the femoral head and the acetabulum and replacing these surfaces with a metal-on-metal ball-and-socket implant [45, 46]. The bone stock retaining nature of hip resurfacing surgery is also providing a push for some surgeons to use HRA as an alternative to THA in elderly patients [47] (Figure 1.8).

Metal-on-metal hip resurfacing is a relatively recent procedure (developed in the mid 90's by McMinn [48] and Amstutz [49]); thus, there is little data available regarding the long term performance of the designs [45]. Quesada et. al. [50] summarize a number of advantages of hip resurfacing procedures including an increase in range of motion, lower dislocation rates, increased levels of patient activity, more normal gait patterns, and ease of revision. The larger bearing surfaces of HRA designs also significantly decrease the rate of surface wear relative to conventional hip implants. The overall short and mid-term performance of hip resurfacing procedures is promising $[45,47,50]$. 


\subsection{1 - Current HRA Designs}

The hip resurfacing implant is designed to mimic the native hip joint. Both components are almost always manufactured from a cobalt-chrome alloy manufactured precisely to provide the best surface wear characteristics possible. The joint diameter is roughly $50 \mathrm{~mm}$, but this is a patient specific parameter (implant head sizes are manufactured in size increments to provide the most accurate fit for each patient). The acetabular component is a metallic cap designed to be pressed into a reamed dish in the patient's pelvic bone. The femoral component is a hollowed portion of a sphere (approximated one-half to three-quarters of a sphere) that is fitted over the bone of the femoral head. Special reaming and shaping tools are used to prepare the femoral head for proper fit of the implant. Most designs also include a peg, or stem, extending from the axis of the femoral component (Figure 1.9).

Traditionally, both the femoral and acetabular components in HRA were implanted using an acrylic bone cement, which helps to evenly distribute the loads between the implant and the bone. Bone cement is a synthetic polymer, poly-methyl methacrylate (PMMA), that was designed to fill or join gaps in bone. It has also found common application as a filler between bone and implant surfaces. The cement has a low Young's modulus (between that of cortical and trabecular bone) and therefore acts as an elastic buffer zone between the stiff metal implant and porous or irregular bone surfaces. The polymer is provided to surgeons in two components: a polymeric initiator often in powered form, and a 

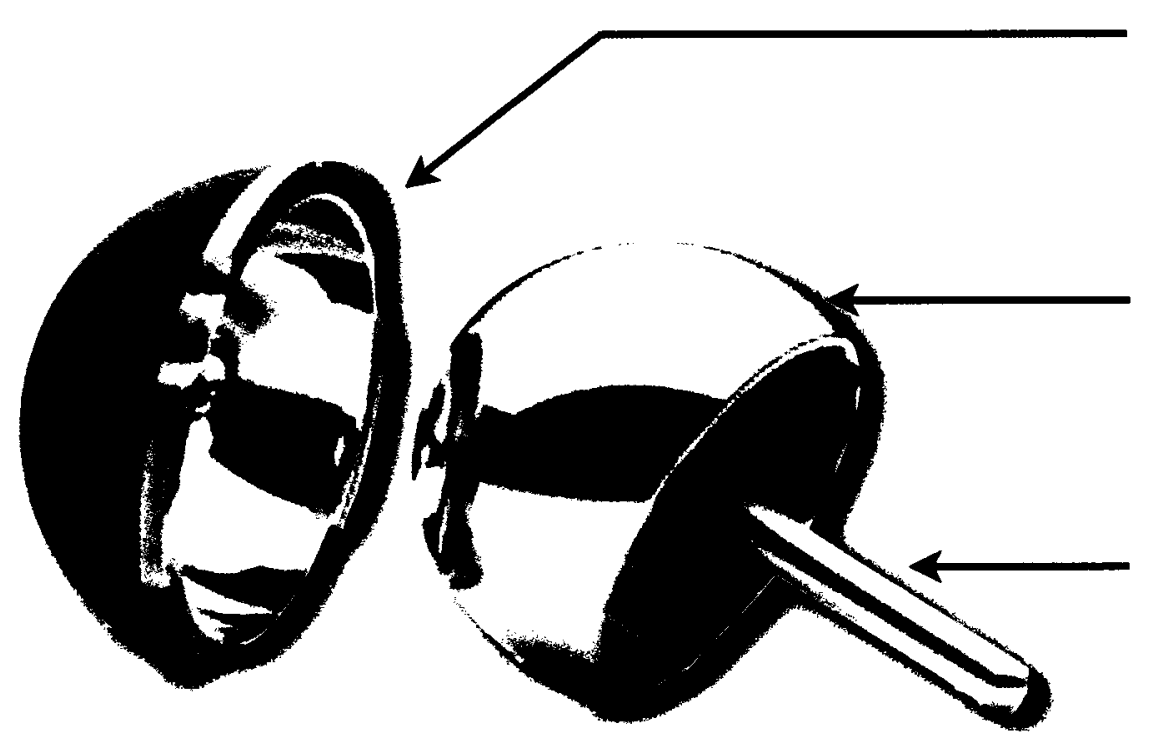

Acetabular cup

Femoral cap

Metaphyseal peg

Figure 1.9: Principle components of a hip resurfacing implant (cemented, Zimmer Durom $^{\mathrm{TM}}$ ) [51].

liquid MMA accelerator. Mixing the two components begins a short setting process where the viscosity of the mixture gradually increases. Surgeons can manually pressurize the cement, in a putty form, onto the reamed bony surface before applying the implant or can coat the inner surface of the implant with the mixture before fitting it onto the bone. The setting process is exothermic and therefore results in an interface temperature increase of between $10^{\circ} \mathrm{C}$ and $30^{\circ} \mathrm{C}$ for 2 to 5 minutes [52]. Consequently, the bone cement is known to cause thermal damage to surrounding tissue and may induce bone tissue necrosis $[53$, 54]. In addition, the cement layer prevents bone in-growth on the implant surface, preventing long term integration in the host bone.

Most current hip resurfacing implants (Figure 1.10) involve hybrid designs where the implanting procedure on the acetabular side has been changed to a 

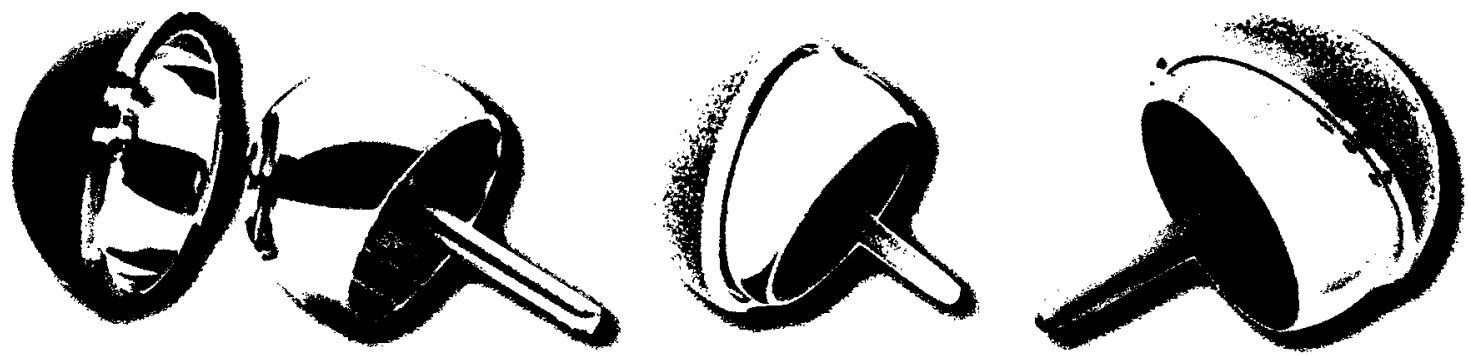

Figure 1.10: Examples of modern metal-on-metal hip resurfacing implants. From left to right: Zimmer Durom ${ }^{\mathrm{TM}}$, Depuy ASR ${ }^{\mathrm{TM}}$, Birmingham Hip ${ }^{\mathrm{TM}}$. Most designs are very similar. All threc implants shown are of a hybrid nature (cementless acetabular, cemented femur). Some designers offer both cemented and cementless versions. Surface treatment varies between cemented and cementless implants. Cementless implants are treated to encourage bone ingrowth on the surface. Some implants introduce fins or longitudinal spines in an effort to increase stability $[51,59,60]$.

press fit design without the use of acrylic bone cement. This approach has shown better clinical results than cemented acetabular designs [55]. Recently, it has been suggested that an entirely cementless design (where neither the femoral component nor the acetabular component require the use of bone cement) may provide better long term results than designs that require cementation of the femoral component. Clinical experience with conventional THA has shown that uncemented femoral stem designs provide better long term results than their cemented counterparts $[56,57]$. Therefore, some of the implant manufacturers have started to design and sell cementless femoral resurfacing components.

The current femoral component designs of HRA use the metaphyseal stem (peg) almost exclusively for alignment purposes [58]. In cases where the femoral component is cemented and a cement mantel forms around the stem, it bears more load. Little et al. [61] suggest that this increased stem loading through cementation may increase bone stress shielding, particularly in the femoral head 
and neck, thereby promoting narrowing of the femoral neck (Figure 1.11), which may lead to fracture. Any form of load bearing that decreases the strains/stress felt by the bone tissue in the femoral head during anatomical loading may induce resorption of this bone; however, it has been shown that such peg loading and this altered stress distribution can be beneficial for patients with large femoral bone defects or small femoral components [58]. The effect of stem fixation on stress shielding of uncemented femoral components is not well understood. In addition, cementless components must be anchored sufficiently to ensure rigid fixation of the component. Micromotion between the component and the bone tissue surface will prevent bone integration with the component, and may lead to

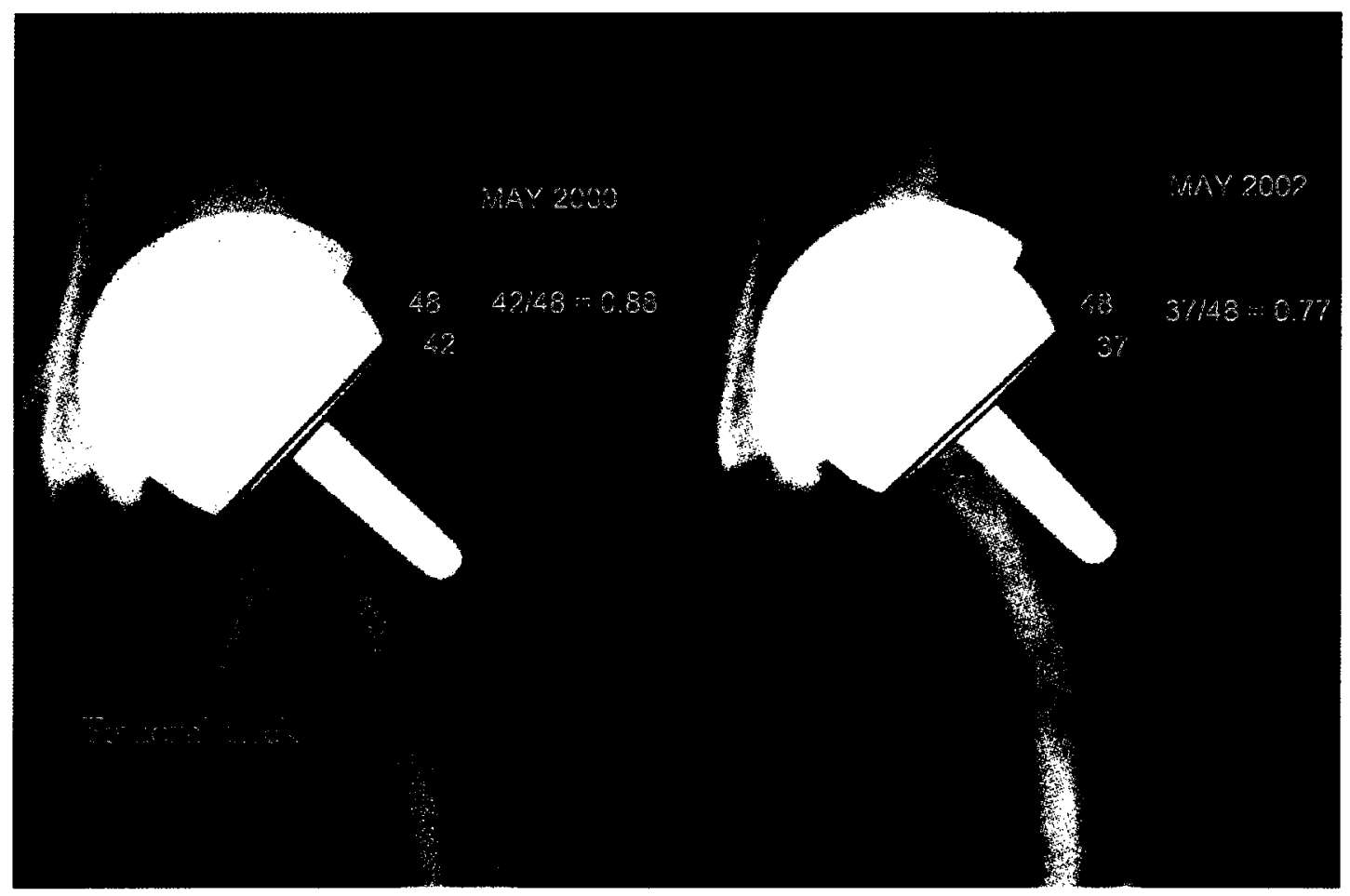

Figure 1.11: Femoral neck narrowing. X-ray images taken from a study performed by Spencer investigating changes in the diameter of the femoral neck after cemented hip resurfacing [62]. Reduction is measured as a ratio of the implant lip diameter (in pink, on the implant) to the diameter of the femoral neck directly adjacent to the implant. 
stress concentrations, bone degeneration, and aseptic loosening of the implant [63].

\section{4 - FEA in Bone Remodelling and Arthroplasty}

Finite element analysis (FEA) is a powerful design tool. When coupled with an algorithm to simulate adaptive bone remodelling, FEA can potentially aid in the design of orthopaedic implants. Specifically, a bone adaptive finite element model can provide insight into how living bone may react to the presence of an implant prior to in vivo or clinical testing. Some authors suggest that FEA cannot be used to accurately predict the clinical performance of an implant; however, they do not deny the usefulness of such an approach as an iterative design tool and a predictor of implant loosening [64 - 66].

Recently, advanced finite element models have been applied to a wide array of orthopaedic design challenges. These include, but are certainly not limited to: total hip arthroplasty [67], shoulder joint arthroplasty [68], dental implants [69], cemented hip resurfacing arthroplasty [15], and even limb replacement prosthesis [70]. In many cases, researchers choose to include bone's adaptive properties into finite element analyses, which requires the implementation of a numerical algorithm to simulate bone adaptation.

Similar to the objective of this thesis, the study performed by Gupta et al. [15] investigated bone remodelling around a resurfaced femoral head; however, their analysis focused on cemented implant. Their full 3D model was generated 
from qCT scan data of the proximal half of a human femur. Ten-node tetrahedral elements were used to mesh the implant, the femur, and a thin layer of PMMA cement that was created between the inner cap of the implant and the bone. No cement was present between the peg and the bone. The elastic modulus of bone was calculated with Equation 1.2 using local density values obtained from qCT data and a Poisson's ratio of 0.3 was used throughout. Simplified loads representing walking and stair climbing were applied to the head of the femur and all nodes at the distal end of their model were rigidly constrained. By implementing a computational routine to model density changes (representing bone adaptation) Gupta et al. were able to investigate remodelling under the cemented resurfacing implant.

There is currently no study that uses the approach presented by Gupta et al. to analyze uncemented femoral resurfacing implants and investigates the effect of fixation and surface interaction on the remodelling of a resurfaced femoral head.

\subsection{1 - Computational Model for Bone Adaptation}

In 1993, Weinans and Huiskes [71] applied a generalized algorithm for bone remodelling after uncemented total hip arthroplasty. It was developed from a variety of different hypotheses proposed at the time. Bone adaptation was governed using a ratio of strain energy density (U) and the local apparently density $(\rho)$. This defines the remodelling stimulus as: 


$$
S=\frac{U}{\rho}
$$

The signal responsible for bone resorption or formation is therefore assumed to be the difference between the actually stimulus level, S, and some reference stimulus, $S_{\text {ref. }} \quad$ It is also assumed that there exists a form of "lazy zone" [72] where no remodelling occurs. This zone is defined by Equation 1.4 and is illustrated in Figure 1.12.

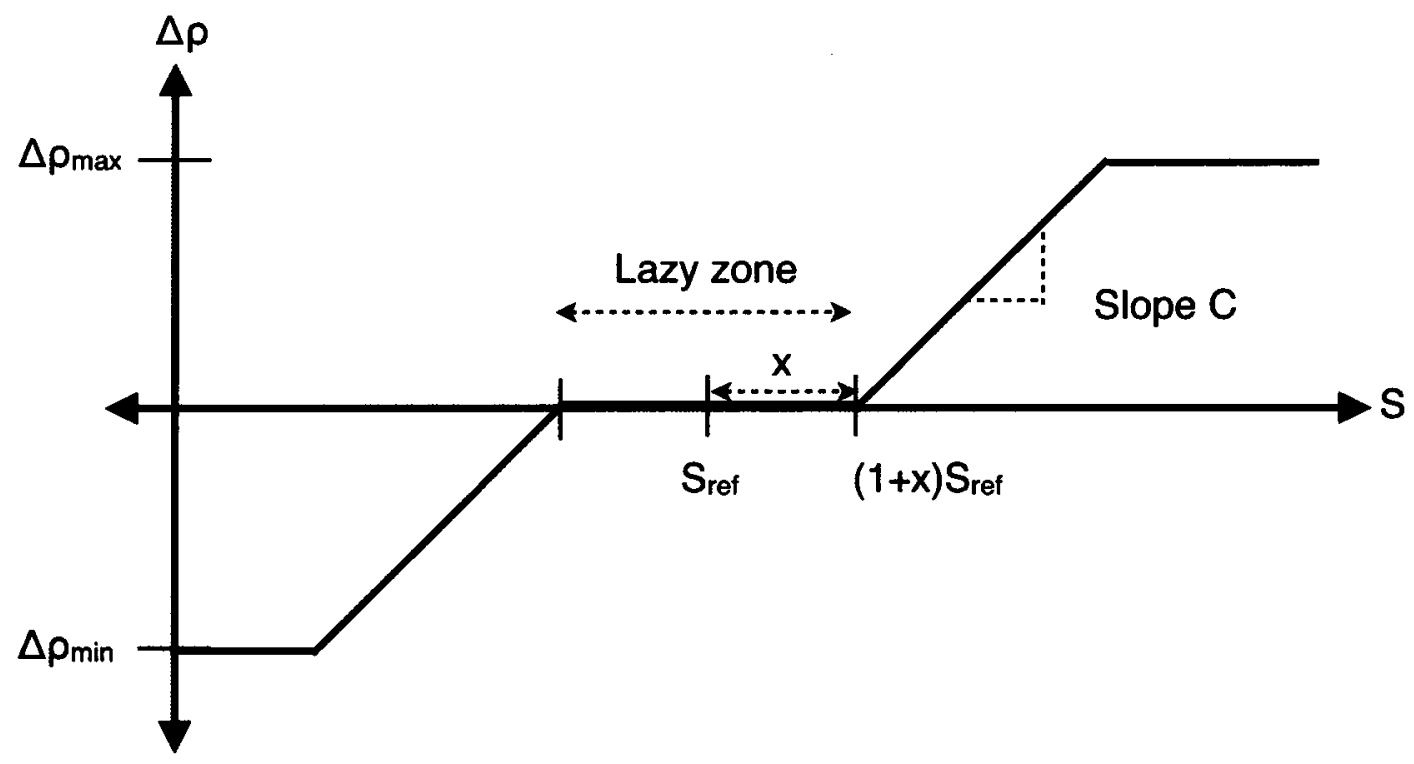

Figure 1.12: Graphical representation of the bone remodelling algorithm. The reference stimulus level, Sref, defines the baseline stimulus level for the algorithm. Any remodelling stimulus, $S$, outside the pre-defined lazy zone defined by $x$, will induce a change in bone density, $\Delta \rho$, governed by the the remodelling rate constant, $C$. In this example, upper and lower limits are placed on the absolute change in density. 


$$
(1-x) S_{\text {ref }} \leq S \leq(1+x) S_{\text {ref }}
$$

The actual change in local bone density is defined by a remodelling rate constant, $\mathrm{C}$, that is unique to each model or simulation and represented by the slope of the inclined sections in Figure 1.12. Sharma et al. [68] chose to vary their remodelling parameter empirically in order to determine the most effective value for their work regarding the development of a bone remodelling simulation for investigating glenoid prosthesis loosening. The constant can be seen as a feedback amplifier that linearly increases or decreases the rate at which bone adapts. Implementing the remodelling rate constant and lazy zone into a single set of conditional equations gives the general governing equations for bone adaptation, Equation 1.5a, as adapted from Weinans [71].

$$
\frac{d \rho}{d t}=\left\{\begin{array}{ccc}
C\left[S-(1+x) S_{r e f}\right] & \text { for } & S \geq(1+x) S_{r e f} \\
C\left[S-(1-x) S_{r e f}\right] & \text { for } & S \leq(1-x) S_{r e f} \\
0 & \text { otherwise }
\end{array}\right.
$$

For the purpose of this study, the equations were implemented into a discrete step based analysis; thus, the time dependent derivative can be written as a $\Delta \rho / \Delta t$. Furthermore, $\Delta t$ can be set equal to unity for each step, giving: 


$$
\Delta \rho=\left\{\begin{array}{ccc}
C\left[S-(1+x) S_{r e f}\right] & \text { for } & S \geq(1+x) S_{r e f} \\
C\left[S-(1-x) S_{r e f}\right] & \text { for } & S \leq(1-x) S_{r e f} \\
0 & \text { otherwise }
\end{array}\right.
$$

Eq. $1.5 b$

Equation $1.5 \mathrm{~b}$ can be implemented in an iterative finite element analysis in order to model bone adaptation in implant-bone models. 


\section{Chapter 2}

\section{Model Development and Validation}

The goal of this thesis was to develop an adaptive model of the proximal human femur to use as a design tool of the femoral components of hip resurfacing arthroplasty prostheses. The model was used to investigate the effects of varying contact parameters at the implant bone-interface on bone remodelling around the implant. It was developed based on a bone density calibrated CT scan (qCT) of a cadaveric femur from which a 3D finite element model with density based material properties was generated. In addition, the theoretical algorithm discussed in Section 1.4.1 was used to simulate bone remodelling. Ultimately, two finite element models of the proximal femur were required to be developed. One model, the "resurfaced model", was shaped to accommodate a resurfacing implant on the femoral head. The second was an "intact" femur model that was used for calibration, validation, and baseline values (initial conditions) for the adaptive simulation.

Figure 2.1 outlines the processes involved in the development of the adaptive resurfaced femur model. The 3D femur model was generated from $\mathrm{qCT}$ scan data obtained from the cadaveric femur specimen. The intact femur and the femur with the hip resurfacing implant were meshed and the bone density based Young's modulus were extracted from the qCT data and assigned to the mesh elements of both models. Finally, the bone's adaptive nature was simulated through the integration of the subroutine implemented within the finite element 
analysis code. In addition, the intact model (prior to remodelling) was validated through mechanical testing of the cadaveric femur. The subsequent sections describe in detail each task performed during the development of the intact and resurfaced finite element femur models.

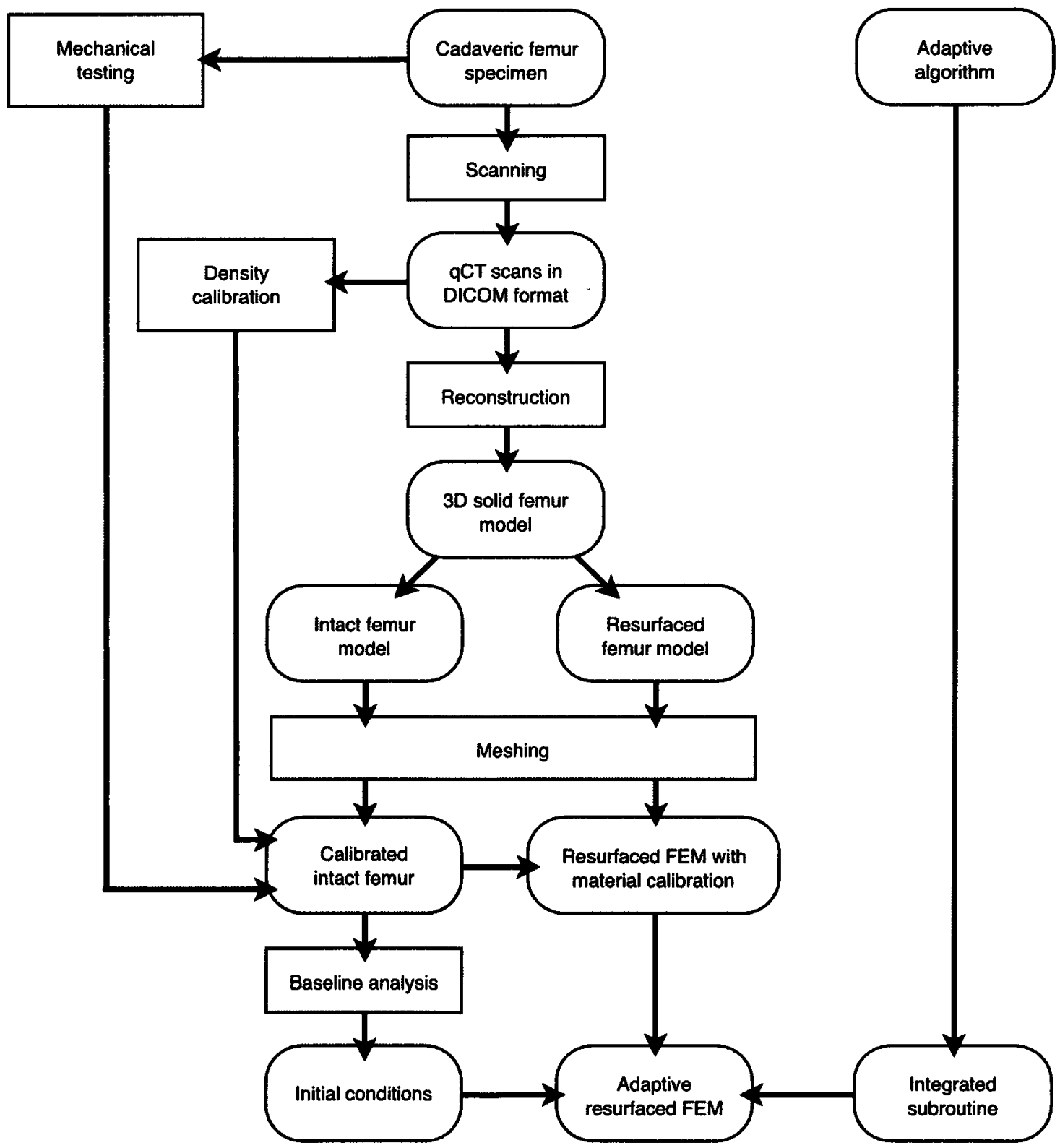

Figure 2.1: Flowchart demonstrating the processes involved in developing an adaptive bone model for the resurfaced proximal femur. 


\section{1 - Constructing the 3D Femur Model}

The modelling of the cadaveric femur can be divided into two tasks: processing the qCT scan images to obtain usable 3-dimensional data and converting this data into a meshable solid part. The method presented here differs significantly from more common segmentation and reconstruction approaches. In segmentation, regions of interest (ROI) are defined either manually or through feature identification algorithms - such as edge detection or graph partitioning. These ROIs are stored for each image, or periodically throughout the stack of images. The resulting array of features represents a series of cross-sections of the physical entity that is to be reconstructed, and these sections can be used directly to create the solid model. In most cases, a number of surface smoothing (noise reduction) algorithms must be introduced before the the model is finalized.

Image segmentation is common for diagnostic and demonstrative models. However, if the model is to be used for finite element modelling or numerical analysis, semi-automatic approaches turned out to be inefficient because great care must be taken to ensure the solid is free of artifacts that may complicate meshing or introduce undesirable effects in loaded engineering models. Manual segmentation proved impractical for the geometry of the proximal femur. Planeby-plane feature identification of the qCT images and solid extrusion is effective for simple geometry - such as the femoral shaft - but poses numerous challenges for more complicated regions like the femoral head or greater trochanter. In an 
effort to simplify the reconstruction process and control the quality of the solid model the approach presented in the following section was used to create a 3dimensional solid model of the proximal portion of a left human femur.

\subsection{1 - Image Thresholding and Obtaining Point Cloud Data}

The first step in the reconstruction approach was 3D feature identification. The goal was to process all images within the CT stack and identify which regions represent relevant femoral bone. The stack of CT scan images was treated as a $3 \mathrm{D}$ volume of black and white voxels, rather than a set of $2 \mathrm{D}$ images. The data matrix was then simplified to contain only voxels that represent relevant femoral geometry. This can be done through "throttling" of the CT image intensity data. It is important to note that this data did not contain sufficient information for direct geometric reconstruction, it was only the first step.

The stack of CT scans, referred to as a series, contains $n$ DICOM images at a pre-defined slice spacing increment, $\Delta z$. The DICOM format (Digital Imaging and Communications in Medicine) is produced by most medical imaging

devices (CT scanners, MRI, etc.). The images in this study were stored at 512 by-512 pixel resolution, with a known scaling factor (from pixels to $\mathrm{mm}$ ) in both $x$ and $y$ directions. Each pixel was assigned an intensity value from the imaging apparatus based on its relative radiodensity. This radiodensity is measured in 
Hounsfield Units (HU), a scaling system based off the linear attenuation coefficients, $\mu$, of distilled water and air (Equation 2.1).

$$
H U=\frac{\mu_{x}-\mu_{\text {water }}}{\mu_{\text {water }}-\mu_{\text {air }}} \times 1000
$$

This formulation is used for all CT scanners calibrated from water. From this, the scanner software is capable of assigning each pixel in a scan slice an intensity value in $\mathrm{HU}$; thus, in addition to storing information regarding spacial dimensions the DICOM file also includes intensity information for each voxel. For the CT scans used, the HU reading for each voxel varied between -1024 and +3071 , with distilled water and air returning approximately 0 and $-1000 \mathrm{HU}$ respectively. It was therefore possible to identify materials (ie: tissues, such as bone) by their expected HU values.

The CT scans obtained for the femur specimens contains 726 images at $500.0 \mu \mathrm{m}$ slice spacing. OsiriX, an open source image processing software developed principally to deal with DICOM images [73] provided a number of powerful tools to view and process CT scans. The 3D "surface rendering" tool allows the user to select an intensity range from which to generate a full 3dimensional isometric surface. By throttling the accepted values to approximately those of bone tissue, it was possible to eliminate all soft tissue 
from the data. The result is a facetted feature including only voxels that meet or exceed the defined $\mathrm{HU}$ value. In this case, OsiriX suggests that bone tissue is isolated at approximately $500 \mathrm{HU}$. It was determined, however, that features of the femoral head were lost at this resolution. Consequently, the range was extended down to $300 \mathrm{HU}$ to accompany the low intensity regions on the surface of the femoral head. Figure 2.2 contrasts the resulting isometric models at 500 $\mathrm{HU}$ and $300 \mathrm{HU}$.

The 3D surface rendering module of OsiriX permits the isometric model to be outputted and stored as a stereolithography file (STL) composed of triangular facets. As seen in Figure 2.2, the model contains both femurs, a calibration phantom (seen in the sagittal views of the rendering) and a noticeable degree of noise artifacts. The reconstruction of one femur without artifacts is described in the following section.

\subsection{2 - Surface Wrapping and Solidification}

The facetted data (Figure 2.2) stored in the STL file was of little use for engineering analysis, and contains a significant degree of artifacts. Pro/ ENGINEER Wildfire 4.0 was used as a modelling tool to build a working solid model of the left proximal femur from the isometric data illustrated in Figure 2.3. This was achieved first by cleaning up the facetted model - removing all unwanted features and any obvious artifacts. Once only desired features remained, the facetted surfaces were "wrapped" using the Pro/E's "Restyle" tool. 


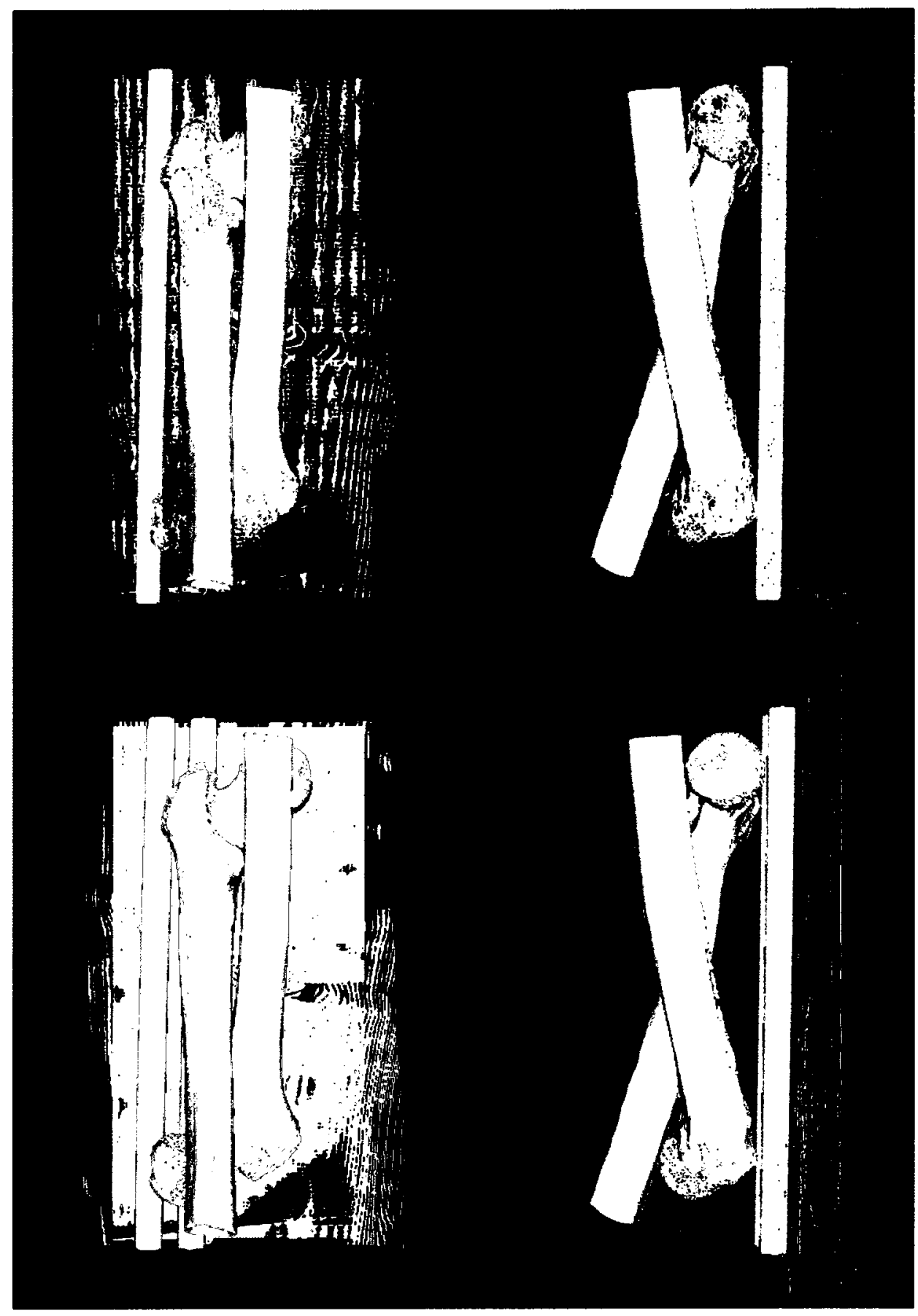

Figure 2.2: OsiriX point cloud data contrasting the resulting isometric models limited to $500 \mathrm{HU}$ (top two images) and $300 \mathrm{HU}$ (bottom two images). The limiting intensity was decreased to $300 \mathrm{HU}$ in the interest of preserving as much much geometric detail as possible, without introducing significant artifacts or noise. 


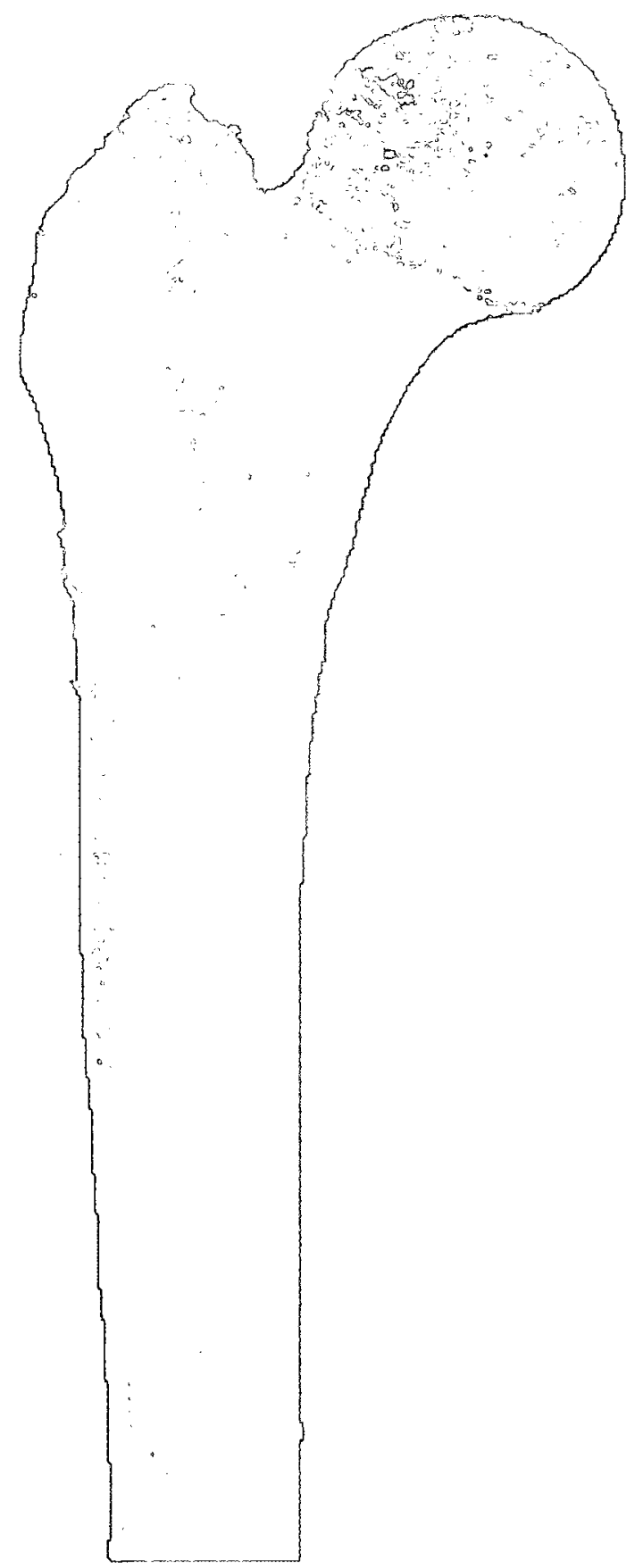

FFigrore 2.3: Cleaned facetted femur as represented in Pro/ENGINEER Wildfire 4.0. Imperfections, such as the voids in the femoral head or excess geometry on the greater trochanter, must be dealt with manually. 
This tool allowed interaction with the nodes and faces of any facetted feature imported as an STL file into Pro/ENGINEER.

The right femur, the calibration phantom, unwanted nodes and faces and other regions not connected with the left femur were identified by visual inspection and deleted. Finally, any large group of facets that were connected with the femur, but did not match the anatomy, were removed. This was the case with two regions on the model: a patch on the distal shaft and another on the anterior aspect of the head. These regions were within direct contact of the right femur during the CT scan. The intensity values in these areas were within the throttled range and during the 3D surface rendering the two femurs were connected at these locations. Once the culling was complete, only facets representing major geometric features of the left proximal femur remained. Any gaps in the facetted surface, either from the deletion process or from the OsiriX output, were patched during the Restyle surface wrap. The final facetted femur (Figure 2.3) contained 560,661 facets where the original file contained over 1.6 million.

As mentioned, automatic extraction of geometric properties from the throttled data proved ineffective. The facetted model from OsiriX did not form a closed surface and it contained numerous unwanted facets that would be tedious to remove manually. For example, the proximal-lateral hemisphere of the femoral head was made of a low density bone tissue. Even at a low HU limit in OsiriX, the surface geometry in this region of the femoral head was spotty (see Figure 
2.3). Consequently, the inner volume of the head contains a number of freefloating facetted tetrahedra that were within the intensity limit. In addition, surface wrapping proved most effective when applied manually. This minimized the risk of capturing surface defects in the final model.

The surface wrapping procedure was performed using the Restyle tool offered in Pro/E. By granting the user control over facetted models, and the ability to interact with the geometry, it was possible to wrap the facetted femur with a number of 3D surfaces. Individual surfaces were defined by parametric curves that were placed along the relevant femoral geometry. By using facets that represent only the relevant surface geometry of the femur, the curves could span gaps in the model, such as the voids mentioned in Figure 2.3. Once the entire surface of the femur was defined by the parametric curves, these curves were used to bound 3D Coons patches, which ultimately forms a surface quilt modelling the geometry of the intact femoral model. Figure 2.4 illustrates the surface patching procedure as performed using Pro/E's Restyle tool.

There proved to be no generalized quantitative technique by which to compare the finalized surface geometry to that of the facetted model. Because the facets do not form a closed volume, or even a closed surface, relative volume comparisons are impractical. At best, the magnitude of offset between facets and an adjacent surface can be measured manually in regions of interest. This task was performed throughout the patching procedure to ensure that the final surface geometry was within a decided tolerance. Facets in the region of the femoral 

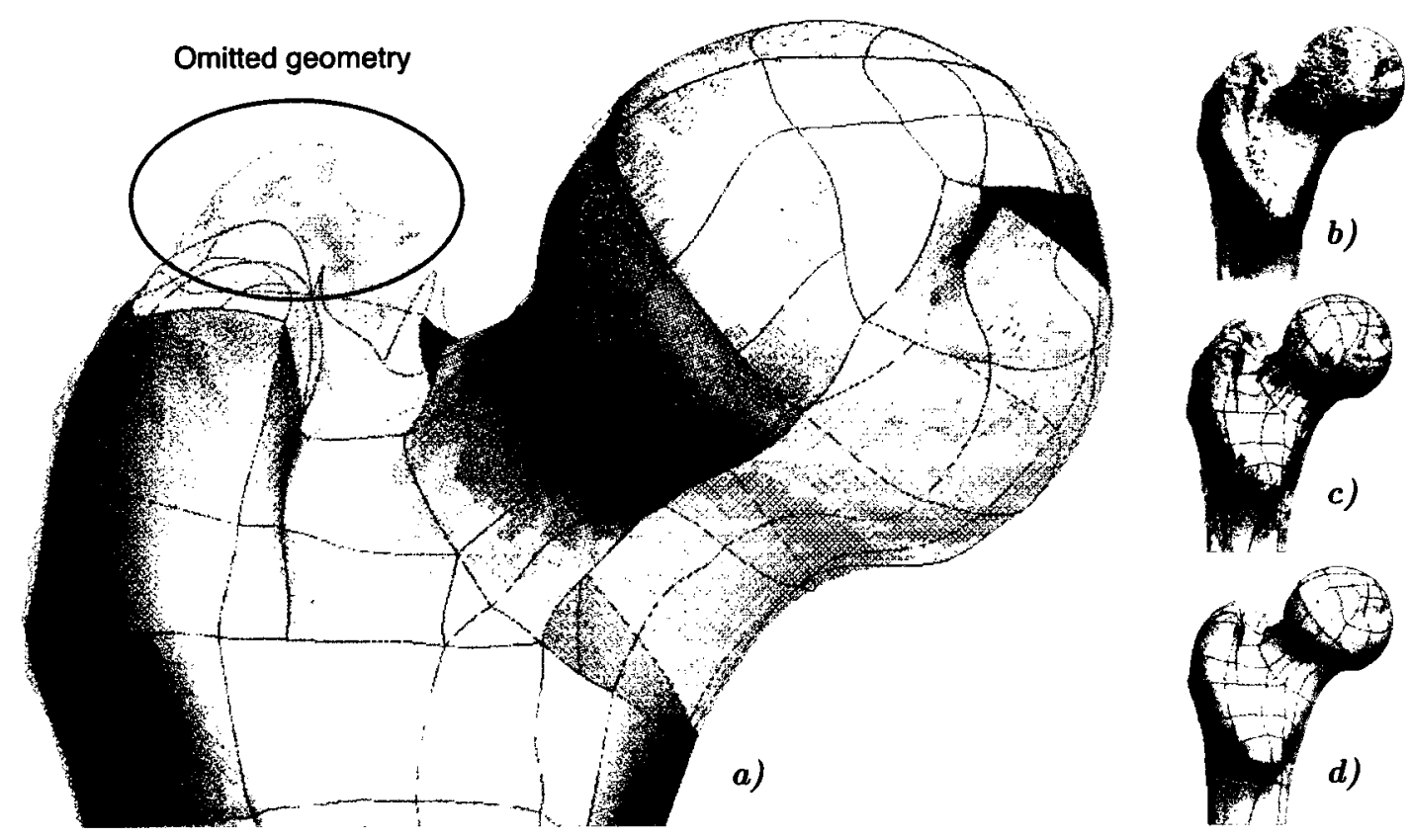

Figure 2.4: Preparation process for the closed surface model of the femur. (a): Surface wrapping progress using Restyle facet interaction. (b): Cleaned facetted model of the left proximal femur. (c): Closed surface features layered on the facetted model. (d): Surface features with facets suppressed. The complex geometry on the proximal greater trochanter was intentionally omitted during the wrapping procedure.

head and neck were allowed to be within $0.5 \mathrm{~mm}$ (the lowest dimension of CT scan resolution) of the nearest surface patch. This distance was measured explicitly as the normal distance from a point (facet vertex) to the surface in question. Certain features, such as the most superior point on the greater trochanter, were ignored entirely (Figure 2.4). Features such as this, that play little to no role in the structural response of the loaded femur, were left out to simplify meshing.

The final surface model was a quilt of 106 individual surfaces modelled by 167 splines. Any closed region can be "solidified" into a solid part in Pro/E, thus, the surface was closed with a level plane at the distal end of the femoral 
shaft and converted into a solid part. The finalized femoral model was exported in STEP format (.stp) and imported into Patran for meshing.

\section{2 - Designing and Fitting the Implant Model}

In order to investigate the influence of femoral hip resurfacing implants, the solid femoral model developed in Section 2.1 (henceforth referred to as the "intact" femur model) was fitted with a resurfacing implant. It was therefore necessary to construct a model of a cementless femoral side resurfacing implant resembling implants currently on the market. Once the implant model was generated, the head of the intact femur was shaped to fit the inner geometry of the implant model.

\subsection{1 - Femoral Resurfacing Implant Model}

The basic design principles of femoral side resurfacing implants were introduced in Section 1.3.1. Designs vary only slightly between manufactures and no particular design has demonstrated superior clinical performance. For the purpose of this thesis, Biomet's ReCapß implant was used as a reference model, because this implant is available as both a cemented and uncemented implant. This will allow direct comparison between uncemented and cemented implants in the future. The surgical techniques for implanting the device, the sizing information, and the X-ray templates are available on Biomet's website [74]. 
Implant sizing was performed as outlined in the surgical procedures document available through Biomet [75]. The circumference of the intact femoral head must be matched to the provided implant template. In this case, four three dimensional implant models of varying head diameters, $50 \mathrm{~mm}$ through $53 \mathrm{~mm}$ at $1 \mathrm{~mm}$ increments, were created (in Pro/E) using the X-ray template provided by Biomet (Figure 2.5a). Completed models were test-fit on the intact femur model. The model sizing that best matched the surface geometry of the intact femoral head was selected as the appropriately sized model; in this case, $50 \mathrm{~mm}$ (Figure $2.5 \mathrm{~b}$ and $\mathrm{c})$.

\subsection{2 - Alignment of the Implant Model}

The femoral implant was aligned as per Dr. Gross' procedure in Biomet's ReCap® surgical technique document [75]. The angle of the metaphyseal stem is

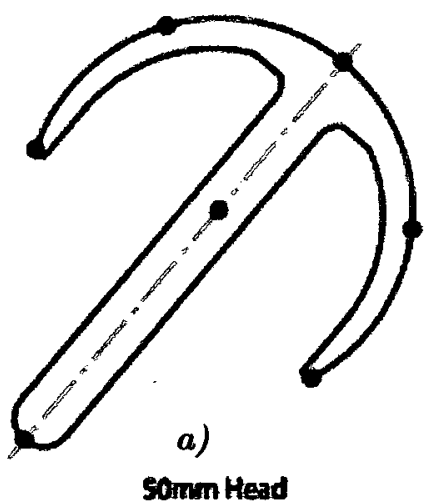

somm Head
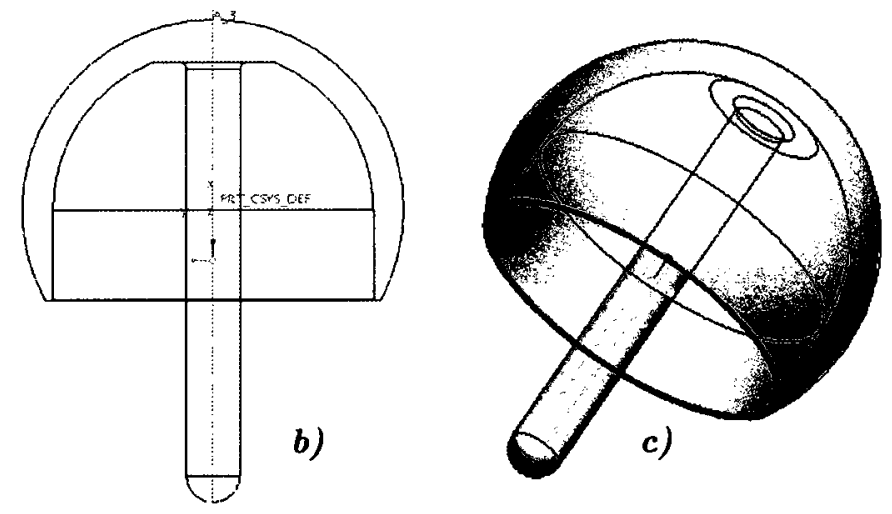

Figure 2.5: Implant model design. (a): X-ray template for a 50mm femoral head component [75]. (b): Cross-sectional sketch developed from the $x$-ray template. This sketch was drawn at four external diameters (head sizes): (50mm, 51mm, 52mm, and 53mm). (c): Finalized $3 D$ resurfacing implant model. 
governed by the angle of the neck (in both coronal and transverse planes). An effort to maintain the center of rotation of the femoral head by matching it to that of the implant model is not only crucial for the clinical success of the implant, but ensures that corresponding physiological loads applied in the FE analysis between the intact and resurfaced models remain comparable (Figure 2.6).

In addition to the implant part, a third solid volume representing a surgical reamer was generated and aligned with the femoral head. Similar to the surgical procedure, the "reamer" was used to ensure that the geometry of the femoral head matches that of the implant. After the implant and the reamer
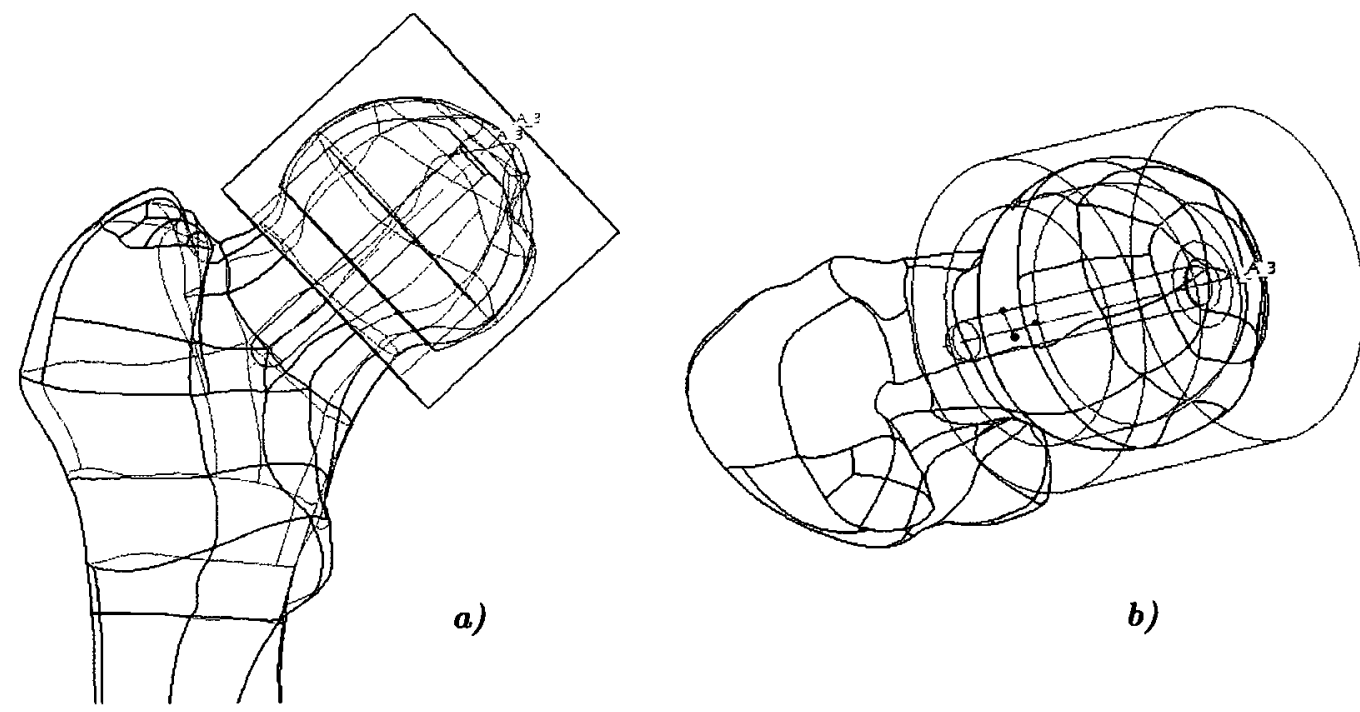

Figure 2.6: Alignment of the implant model on the intact femur. (a): Posterior view, coronal plane. The red highlights show the geometry of the implant. (b): Superior view, transverse plane. The red highlights show the geometry of the reamer part. This part was used to remove all unwanted bone geometry, leaving the femoral head shaped to match the implant's inner surface. 
were aligned with the intact femur, the solid models were exported in the STEP format prior to performing Boolean operations.

\section{3 - Assembly and Meshing}

Meshing of the femoral model was performed using Patran 2008 R2. The STEP files from Section 2.2 were imported, the femur was shaped using the reamer model to accommodate the implant using Boolean operations, and the models (both intact and resurfaced femur models) were meshed. Patran's compatibility with Abaqus allowed for direct creation of input files (.inp) that can be read by the Abaqus processor, or imported into Abaqus/CAE as an orphan mesh. An orphan mesh is an Abaqus mesh whose material properties are open for assignment in CAE, but whose geometric properties cannot be changed.

\subsection{1 - Boolean Operations}

After completing all the required alignments of the reconstructed intact femur model, the implant model, and the reamed model in Pro/ENGINEER, the models were imported into Patran to perform the required Boolean operations and generate the mesh of intact femur and the resurfaced femur (Figure 2.7) which will be required for the validation and simulation. Since the intact model served as the baseline conditions (i.e. baseline strain energy density) for the bone adaptive remodelling simulation, it was necessary that the mesh and element numbering in the reamed femur was identical to the mesh and element numbering 

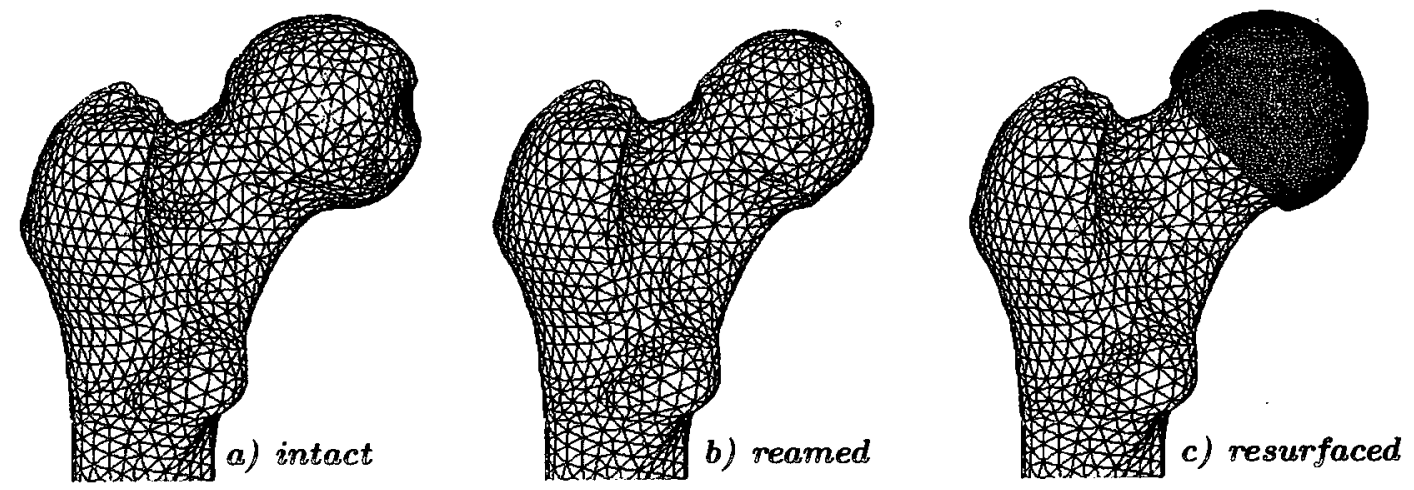

Figure 2.7: Three principal modelling phases. Boolean operations were performed on the intact femur (a) using the reamer part to obtain the reamed femur geometry (b). The final resurfaced femur model (c) is obtained by assembling the reamed femur with the implant model.

in the corresponding regions of the intact model. It was therefore apparent that the intact femur must be constructed using the reamed femur, where the reamed femur can be obtained as:

$$
\text { reamed }=\text { intact }- \text { reamer }
$$

Eq. 2.2a

The other geometric component required to complete the intact model is the bone that was just removed by the reamer part in Equation 2.2a. This feature is identified by the intersection of the reamer and the intact femur:

$$
\text { removed bone }=\text { intact } \cap \text { reamer }
$$


The final full femur model was created as the assembly (not union) of the removed bone geometry and the reamed femur. By assembling the two independent geometric features, the final geometry includes a shared surface at the location where the implant component will sit in the resurfaced femur model. This shared surface allows for the region of the intact mode represented by the reamed model to use the same mesh as the reamed model itself - which also ensured matching element numbers. The resurfaced femur model, Figure 2.7c, was assembled directly from the reamed model and the implant.

\subsection{2 - Mesh Design}

The assembled intact femur model was meshed with dependencies between the reamed femur and the removed bone parts. A shared-node interference mesh was generated at the interface surface (Figure 2.8c). This allowed the mesh of the reamed femur part to be reused in the resurfaced model while still maintaining a continuous mesh for the intact model. Because the nodes were shared between both parts of the mesh, there was no need for interaction properties or constraints at this interface.

The femur models were meshed using quadratic (10-node) tetrahedrals with a maximum edge length of $3 \mathrm{~mm}$. Interior elements were coarsened in the femoral shaft, and lateral of the implant peg towards the greater trochanter, to reduce the overall element count. The remodelling subroutine became unstable with drastic changes in element size (discussed in Section 2.5). Consequently, a 

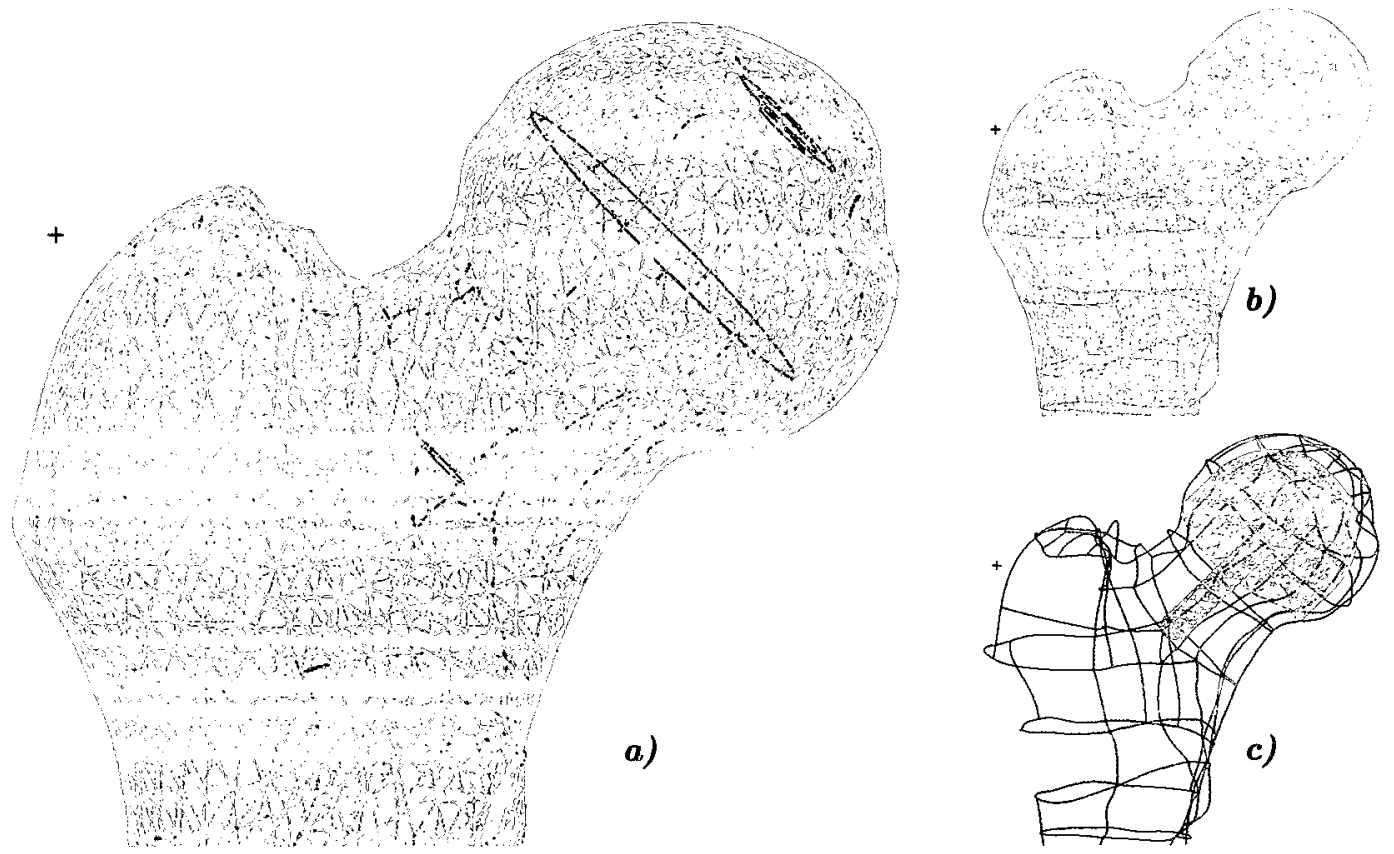

Figure 2.8: Patran tetrahedral mesh of the femur models. (a): Intact femur assembled from the meshed reamed femur model and the material removed during the reaming process. (b): Reamed femur mesh. (c): Interference mesh of the intact femur. Nodes are shared along the boundary surface creating a continuous mesh assembly with no interactions or constraints.

constant seed size was applied over the surface of the entire reamed model. Element size was limited by computation time; however, the mesh needed to be fine enough to capture sufficient material information (densities) from the $\mathrm{qCT}$ scans and to accurately simulate the effects of bone remodelling.

In contrast to the intact femur, the implant model was meshed independently from the reamed femur, since the constraints and interaction properties were applied to the implant-bone interface surface, and the material properties of the implant were constant. Therefore, it was not necessary to create a continuous mesh for the resurfaced femur model. Because all loads and 
interactions were assigned in Abaqus CAE, the implant model was meshed in Abaqus, rather than Patran like all other parts. This provided greater flexibility than working with an orphan mesh. Both the intact femur and the reamed femur models were imported as orphan meshes from Patran output.

\subsubsection{1 - Element Type: C3D10M}

All meshes generated for the final femoral models (both resurfaced and intact) are composed of modified 10-node tetrahedral elements - C3D10M in Abaqus. This is a three-dimensional solid quadratic stress/strain tetrahedral. Each tetrahedral is defined by ten nodes: four vertex nodes and six mid-side nodes. The element uses four integration points in its stiffness matrix - each integration point contains independent material point information. The modified formulation (M) provides the element with hourglass control and a resistance to contact overclosure (element nodes from one surface penetrating the surface of its contact pair) [76]. Tetrahedral elements are better suited to modelling the complex geometry of the proximal femur. The C3D10M element is a common choice for finite element analysis of different orthopaedic devices and organic structures $[77-80]$.

\subsubsection{2 - Mesh Summary}

Table 2.1 summarizes the element and node information for the intact femur model and the resurfaced femur model. Note that nodal information was 


\begin{tabular}{|c|c|c|c|c|c|}
\hline \multicolumn{1}{|c|}{ Model } & Type & Elements & Nodes & $\begin{array}{c}\text { Min. Inside } \\
\text { Angle }\end{array}$ & $\begin{array}{c}\text { Max. } \\
\text { Inside } \\
\text { Angle }\end{array}$ \\
\hline Intact Femur & C3D10M & 28424 & 44128 & $14.5^{\circ}$ & $140^{\circ}$ \\
\hline Resurfaced Femur & & & & & \\
\hline Femur (reamed) & C3D10M & 24049 & 44128 & $14.5^{\circ}$ & $140^{\circ}$ \\
\hline Implant & C3D10M & 39407 & 65323 & $8.0^{\circ}$ & $150^{\circ}$ \\
\hline
\end{tabular}

Table 2.1: Mesh information for resurfaced and intact femur models.

maintained between the intact and reamed femur models. The intact and reamed femur meshes were imported as orphan meshes from Patran into Abaqus. As mentioned earlier, all geometric operations are prohibited on orphan mesh features. All pre-processing, such as assigning boundary conditions, interactions, and loads was performed in Abaqus (Section 2.7). The elements of the femoral models (intact and reamed) were assigned material properties using intensity information gathered from the qCT scans.

\section{4 - Assigning Material Information}

Referring to Equations 1.1 and 1.2, it is apparent that the elastic modulus of bone can be estimated through density. Density information for the femoral model was stored within the CT scan series as image intensities. This section addresses the process by which this intensity information was transformed into density data, and outlines how material properties were assigned to the elements of the intact femur model. 


\subsection{1 - Calibration of CT Intensities}

As outlined in Section 2.1.1, the CT scan series is a stack of images with three-dimensional coordinate information, and intensity data stored in Hounsfield Units (HU). The X-ray image intensity is proportional to the density of materials being scanned. It was possible to determine an approximate relationship between the $\mathrm{HU}$ value of a particular voxel (or region) and the density of that region through the inclusion of a calibration device in the scan itself.

\subsubsection{1 - The Calibration Phantom}

The device, a CT calibration phantom, was located below the femur specimens during the scanning process. The phantom used in this study was a Mindways Software, Inc. Model 3 CT Calibration Phantom, which contained 5 rods of different reference materials, with known reference densities (Table 2.2), embedded in a plastic support matrix (Figure 2.9). The phantom was placed

\begin{tabular}{|c|c|c|}
\hline Reference Rod & Eqv. $\mathrm{H}_{2} \mathbf{O}$ Density $(\mathbf{m g} / \mathbf{c c})$ & Eqv. $\mathbf{K}_{\mathbf{2}} \mathbf{H P O}_{\mathbf{4}}$ Density $(\mathbf{m g} / \mathbf{c c})$ \\
\hline $\mathrm{A}$ & $1012.25 \pm 2.27$ & $-51.83 \pm 0.12$ \\
\hline $\mathrm{B}$ & $1056.95 \pm 1.94$ & $53.40 \pm 0.10$ \\
\hline $\mathrm{C}$ & $1103.57 \pm 1.69$ & $58.88 \pm 0.09$ \\
\hline $\mathrm{D}$ & $1119.52 \pm 1.82$ & $157.05 \pm 0.26$ \\
\hline $\mathrm{E}$ & $923.20 \pm 2.12$ & $375.83 \pm 0.86$ \\
\hline
\end{tabular}

Table 2.2: Phantom reference material composition. 


\section{ans}
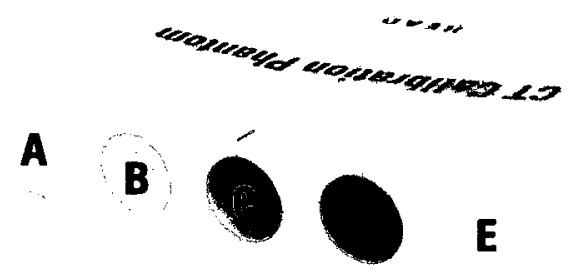

Figure 2.9: CT calibration phantom. Placed below the femur specimens during the CT scan to provide reference information for density calibration. Model 3, Mindways Software, Inc. [81].

longitudinally in the CT scanner such that each CT slice contained a crosssection of the 5 rods.

\subsubsection{2 - Density Estimation}

The process of estimating the density of a particular region of interest (ROI) from reference material was adapted from the documentation which was shipped with the calibration phantom by Mindways Software, Inc. [81]. The following nomenclature is pertinent to this section:

$\rho_{\mathrm{K} 2 \mathrm{HPO} 4}$

$\mathrm{K}_{2} \mathrm{HPO}_{4}$ equivalent density of material within the ROI 


$\begin{array}{ll}\mu_{\mathrm{ROI}} & \mathrm{CT} \text { intensity value (in HU) of the ROI } \\ \rho_{\text {water }} & \text { Water equivalent density of the material within the ROI } \\ \sigma_{\mathrm{ref}} / \sigma_{\mathrm{CT}} & \text { Imaging technique-specific parameter } \\ \beta_{\mathrm{ref}} / \beta_{\mathrm{CT}} & \text { Imaging technique-specific parameter }\end{array}$

The density of the material contained within a specified region of interest is referred to as the $\rho_{\mathrm{K} 2 \mathrm{HPO}}$ equivalent density. The material is characterized by identifying the equivalent concentration of $\mathrm{K}_{2} \mathrm{HPO}_{4}$ dissolved in water that would demonstrate the same attenuation properties as the unknown material. The imaging technique-specific parameters are variables used to govern the relationship between CT image intensity and equivalent $\mathrm{K}_{2} \mathrm{HPO}_{4}$ density.

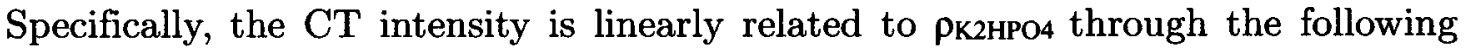
relationship:

$$
\mu_{R O I}=\sigma_{C T} \cdot \rho_{K 2 H P O 4}+\beta_{C T}
$$

Equation 2.3 is the generic governing relationship between equivalent $\mathrm{K}_{2} \mathrm{HPO}_{4}$ density and CT image intensity. The parameters $\sigma_{\mathrm{CT}}$ and $\beta_{\mathrm{CT}}$ are directly related to the imaging parameters that must be determined for the reference materials within the calibration phantom. 


$$
\begin{gathered}
\sigma_{C T}=\sigma_{r e f}-0.2174 \\
\beta_{C T}=\beta_{r e f}+999.6
\end{gathered}
$$

Eq. 2.4b

The reference parameters for the phantom used in this study, $\sigma_{\text {ref }}$ and $\beta_{\text {ref, }}$ are determined through linear regression of Equation 2.5 .

$$
\mu_{R O I}=\rho_{\text {water }}+\sigma_{r e f} \cdot \rho_{K 2 H P O 4}+\beta_{r e f}
$$

This equation is specific to the CT calibration phantom; thus, by providing CT intensity values for each of the 5 known reference materials, the unknown parameters can be determined through linear regression. In this case, OsiriX was used to sample circular regions of interest centered within the reference material rods (Figure 2.10). The mean intensity value was recorded for each sampled region. A total of eight sets of ROI samples were taken incrementally throughout the CT scan stack. The average intensity for each reference rod was calculated and tabulated in Table 2.3. Equation 2.5 was used to determine the values of $\sigma_{\text {ref }}$ and $\beta_{\text {ref }}$ through linear regression. Table 2.4 summarizes the data arrays used in solving the regression problem. In turn, the values of $\sigma_{\mathrm{CT}}$ and $\beta_{\mathrm{CT}}$ were calculated using Equations 2.4a and 2.4b. Finally, the equivalent $\mathrm{K}_{2} \mathrm{HPO}_{4}$ density for any given region of interest with a known CT 


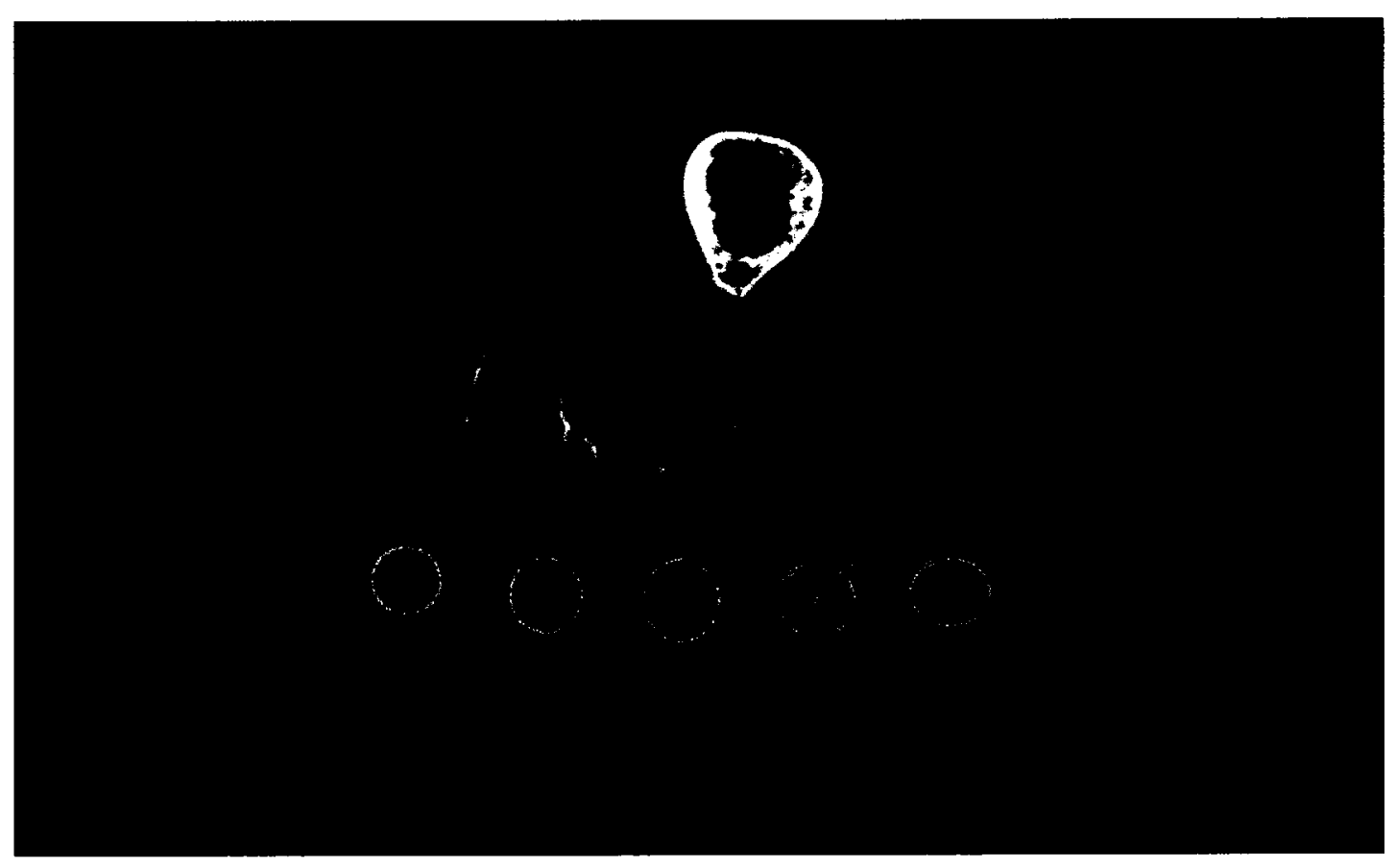

Figure 2.10: Sampliny ROI intensities in OsiriX. A mean intensity value is determined for each region on eight different slices throughout the stack. The overall intensity for each material is determined as the average of the eight readings.

\begin{tabular}{|c|c|c|c|c|c|c|c|c|c|}
\hline & \multicolumn{10}{|c|}{ Slice number } \\
\cline { 2 - 11 } & $\mathbf{1 0 5}$ & $\mathbf{1 6 5}$ & $\mathbf{2 2 5}$ & $\mathbf{2 8 5}$ & $\mathbf{3 4 5}$ & $\mathbf{4 0 5}$ & $\mathbf{4 6 5}$ & $\mathbf{5 2 5}$ & Avg. \\
\hline Rod & \multicolumn{8}{|c|}{ CT number (HU) } \\
\hline A & -95 & -96 & -97 & -95 & -94 & -95 & -93 & -95 & -95.0 \\
\hline B & -65 & -67 & -65 & -64 & -69 & -65 & -69 & -68 & -66.5 \\
\hline C & 207 & 205 & 202 & 202 & 201 & 204 & 208 & 201 & 203.8 \\
\hline D & 412 & 414 & 413 & 419 & 409 & 412 & 418 & 414 & 413.9 \\
\hline E & 590 & 593 & 604 & 592 & 614 & 600 & 594 & 612 & 600.0 \\
\hline
\end{tabular}

Table 2.3: Reference rod intensities. 


\begin{tabular}{|c|c|c|c|}
\hline Reference Rod & $\varrho_{\text {water }}(\mathbf{m g} / \mathbf{c c})$ & OK2HPO4 $^{(\mathbf{m g} / \mathbf{c c})}$ & $\mu_{\text {ROI }}(\mathrm{HU})$ \\
\hline A & 1012.25 & -51.83 & -95.0 \\
\hline B & 1056.95 & 53.40 & -66.5 \\
\hline C & 1103.57 & 58.88 & -203.8 \\
\hline D & 1119.52 & 157.05 & 413.9 \\
\hline E & 923.20 & 375.83 & 600.0 \\
\hline
\end{tabular}

Table 2.4: Data array used for linear regression of Equation 2.5.

intensity is calculated directly from a rearranged form of Equation 2.3.

$$
\rho_{K 2 H P O 4}=\frac{\mu_{R O I}-\beta_{C T}}{\sigma_{C T}}
$$

Eq. 2.6

Using the data in Table 2.4 to solve for $\sigma_{\text {ref }}$ and $\beta_{\text {ref }}$ in Equation 2.5 yeilds 1.857 and -1012.6 respectively. The values of $\sigma_{\mathrm{CT}}$ and $\beta_{\mathrm{CT}}$ are found to be 1.640 and -13.0. From Equation 2.6, this gives the final calibration estimate.

$$
\rho_{K 2 H P O 4}=\frac{\mu_{R O I}+13.0}{1.64}
$$




\subsection{3 - Assigning Element Properties}

To capture the varying density, and consequently the elastic modulus, of the bone tissue throughout the femoral head, each element was assigned a unique density. This requires aligning the solid mesh with the CT scan series and sampling the CT intensity data within each element. Amira 5.2.2, a research software package developed by Visage Imaging ${ }^{\circledR}$ that excels in work related to medical imaging, was used to register intensity data for the intact femur mesh.

Node and tetrahedral information extracted from the Abaqus input file generated by Patran was reformatted and imported into Amira. The CT scans containing the grayscale values were imported into Amira in the original DICOM format. Amira stores both the CT scans and the mesh file as "data objects", which can be visualized, transformed, and manipulated in other ways. Even though the scans were performed such that the z-direction was normal to each slice, Amira can present the user with CT images in any of the three planes. It is also possible to sample intensity data in pre-defined 3D regions of interest.

Once both the mesh data and the CT series are present in the data pool, they must be aligned. Because the femur model was reconstructed directly from the CT data, without scaling or rotation of the geometry, only a linear shift in the z-direction was necessary. This shift originated when 120 scan slices at the distal end of the left femur were ignored during reconstruction. The scan extended well past the length of the specimen and these images did not contain relevant geometric data. Scans were performed every $500 \mu \mathrm{m}$, and so a $60 \mathrm{~mm} \mathrm{z}-$ 
direction translation transformation was applied to the mesh. After the transformation, the CT scans and the mesh geometry were aligned (Figure 2.11).

Amira provides an integrated tool that can access each mesh element (as a tetrahedral ROI) and sample the CT intensity values contained within that region. Attaching this feature ("SampleScalarField") to both the mesh data object and the CT series object tells Amira to create a scalar field of average intensity values for corresponding elements. The output file, in simple text format, was then imported into a Matlab routine (Appendix A) that applies

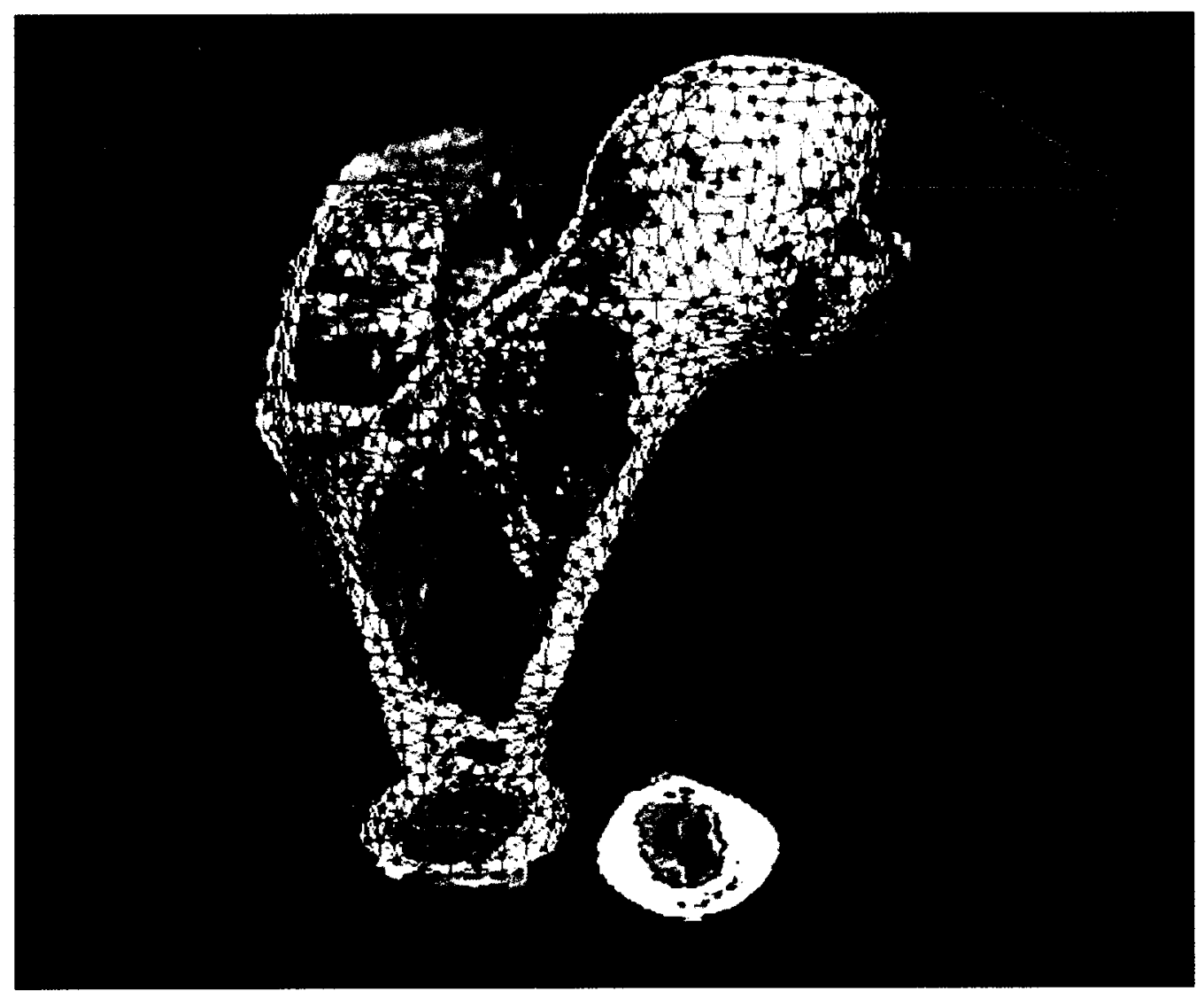

Figure 2.11: Visualization of $C T$ and mesh data in Amira. High intensity regions (white) clearly outline the geometry of the femur specimen. Amira is used to align CT data with the Abaqus mesh, and assign intensity values to each element. 
Equation 2.7 and outputs a formatted file containing density information for each element. This file was used as initialization input in the Abaqus analysis and was imported through a Fortran subroutine in both the intact femur model (for baseline analysis) and the resurfaced femur model. As introduced in Section 1.2.2.2, Equation 1.2 was used to determine the elastic modulus values (Young's Modulus) for each element in the intact femoral and resurfaced model. Poisson's Ratio was assumed to be constant isotropic value of 0.3 , despite its anisotropy [68, 82]. Gupta et al. [82] and Sharma et al. [68] have both successfully modelled bone of the proximal femur using simplified isotropic properties. The implant was assumed to be manufactured from a cobalt-chrome alloy with a uniform modulus of 220GPa and a Poisson's ratio of 0.31 .

\section{5 - Fortran Subroutines}

Abaqus allows users to include custom subroutines, written in Fortran, to enhance the capabilities of analyses in both Abaqus/Standard and Abaqus/ Explicit. The subroutine USDFLD was used to allow an incremental change in material properties over an analysis step. This function provides user access to material point information at the beginning of each increment. It also allows the user to create and access any number of state and field variables for each integration point. Field variables are used to define material information and must be assigned for each call of USDFLD. State variables can be accessed by other Abaqus subroutines, but are also useful for storing information between 
steps. Any value stored in a state or field variable is passed into the USDFLD subroutine at the start of every increment. These values can be updated within the subroutine, and the updated values will be applied in the subsequent step. In addition, state and field variables can be requested as output variables in Abaqus CAE for post-processing. The subroutine is called separately for every integration point of every element; thus, computation times increase quickly with increasing element count or element order.

A USDFLD subroutine was used to import the initial density data determined in Section 2.4.3, calculate the corresponding material elastic modulus from Equation 2.7, and output the reference stimulus signal from the intact femur model. A second USDFLD subroutine was integrated into the FEA in order to simulate bone remodelling by varying the bone density of all relevant elements within the resurfaced femur model. The Fortran code for both routines is listed in Appendix B.

\subsection{1 - Baseline Analysis Subroutine}

The purpose of the baseline analysis was to import material information into the intact femur model and to determine the reference stimulus state for the remodelling analysis. Seven state variables (Table 2.5) and one field variable were defined within the subroutine and the Abaqus input file. The field variable was used as a material dependency; specifically, the bone material modulus was tabulated such that the value of Young's modulus corresponded directly with the 


\begin{tabular}{|c|c|}
\hline Variable & Representative value \\
\hline FV1 & Elastic Modulus (Pa) \\
\hline SDV1 & Elastic Modulus (Pa) \\
\hline SDV2 & Current density $(\mathrm{g} / \mathrm{cc})$ \\
\hline SDV3 & Baseline remodelling stimulus, $\mathrm{S}_{\mathbf{n}}(\mathrm{J} / \mathrm{g})$ \\
\hline SDV4 & Current remodelling stimulus, S $(\mathrm{J} / \mathrm{g})$ \\
\hline SDV5 & Feedback ratio, $[\mathrm{S}-(1 \pm$ Sn) $(\mathrm{J} / \mathrm{g}) \ldots$ zero for baseline case $\}$ \\
\hline SDV6 & Step change in density, $\Delta \mathrm{Q}(\mathrm{g} / \mathrm{cc}) \ldots$ zero for baseline case $\}$ \\
\hline SDV7 & Initialization boolean \\
\hline SDV8 & Baseline density, $\mathrm{Q}_{\mathbf{u}}$ \\
\hline SDV9 & Absolute change in density, $\mathrm{Q}-\mathrm{Q}_{\mathbf{n}}(\mathrm{g} / \mathrm{cc})$ \\
\hline SDV10 & Absolute change in stimulus, S-S $(\mathrm{J} / \mathrm{s})$ \\
\hline SDV11 & Percent change in density \\
\hline SDV12 & Percent change in stimulus \\
\hline
\end{tabular}

Table 2.5: Listing of all state (SDV) and field (FV) variables used in the remodelling subroutine and their associated values. Only variables 1-7 are initiated in the baseline subroutine and among them SDV4 and SDV5 are set to zero.

value of the field variable. For example, if the field variable returned from the subroutine with a value of $50,000,000$ for a specific element, the elastic modulus of that element was set to 50MPa. As mentioned earlier, Poisson's ratio was set to a constant value of 0.3 and was therefor unaffected by changes to the field variable. Table 2.5 identifies the values stored within each state and field variable. The table is generic for both the baseline subroutine and the remodelling subroutine. Only the first 7 variables were used in the baseline case (Table 2.5).

The baseline analysis was run for two steps. The initial step was used to import density data from the initial conditions file (Section 2.4.3) and calculate 
the corresponding elastic modulus for each element. The second step was used to output density and baseline stimulus data for each integration point of every element based on Equation 1.3 in Section 1.4.1. The value of strain energy density or density for any integration point can be requested from Abaqus within the subroutine through use of the GETVRM function call.

\subsection{2 - The Remodelling Subroutine}

The remodelling subroutine was designed for use with the resurfaced femoral model and had a similar program structure to that of the simple baseline analysis subroutine. However, unlike the baseline subroutine, which simply reads the initial conditions, this subroutine performed calculations on the state and field variables, and outputted updated values. The remodelling algorithm was constructed based on the algorithm outlined in Section 1.4.1 through use of conditional statements. The first increment of the Abaqus analysis was used to import the initial condition file generated by the baseline subroutine. State variables were used to store the baseline values of density and the remodelling stimulus at each integration point. The first step of the analysis returned a stimulus field for the bone material that differed from that of the baseline analysis. The stress distribution within the femoral head was influenced by the presence of the metallic implant (see Section 1.2.2.4 with regard to stress shielding) and this difference in stimulus level was what drove the bone remodelling. 
Once the first analysis step was completed, all subsequent subroutine calls were used to check for changes in the reference stimulus and to remodel accordingly. A remodelling rate constant of $4.0 \times 10^{-6}$ ( $\mathrm{C}$ in Equation 1.5) was determined to be the most effective. A smaller rate allows for more stable remodelling (smaller changes in density per step), but increases the number of steps required to reach equilibrium. The lazy zone was defined to be $\pm 10 \%$ of $\mathrm{S}_{\mathrm{ref}}$ $(x=0.10)$. The zone was widened slightly from the validation model (Section 2.5.3) to prevent density oscillation in high strain elements. The bone density was limited to values between $0.01 \mathrm{~g} / \mathrm{cc}$ and $2.5 \mathrm{~g} / \mathrm{cc}$. The minimum value was essential to prevent the collapse of low modulus elements, particularly in analyses with contact, and was also the lower validation limit for Equations 1.1 and 1.2 $[15,16,71]$. The upper density limit was pushed above that recommended by Weinans [71]. The elastic modulus equations are thought to be valid up to densities of $1.8 \mathrm{~g} / \mathrm{cc}$; however, the CT scan calibration returned some element densities as high as $2.03 \mathrm{~g} / \mathrm{cc}$ in the cortex. In order to accommodate these densities, and to allow for limited remodelling of the cortical bone, densities up $2.5 \mathrm{~g} / \mathrm{cc}$ were permitted. This value exceeds the maximum physiological expected in the femur; however, increasing the permissible range allows for limited investigation of regions that may exhibit large increases in density. In addition to the absolute limits placed on the bone density, the step change in density was limited to $0.05 \mathrm{~g} / \mathrm{cc}$ per step. 
Once the parameters governing the remodelling routine were established, implementation was simply a matter of applying Equation 1.5b. Changes to density were reflected directly in changes to the elastic modulus field variable, thus increasing or decreasing local element modulus in an effort to drive the stimulus signal back to reference values. The analysis could be run for an indefinite number of steps with remodelling occurring from step 2 onwards.

\subsection{3 - Subroutine Validation}

Before the subroutine was applied to the femoral model, it was tested in a simplified loading scenario to validate its performance. In its simplest form, the routine reacts to variation in stimulus levels (directly proportional to strain energy) by increasing or decreasing the elastic modulus of the element in order to drive the stimulus signal back to baseline values. A cantilever beam model was used to test the performance of the Fortran routines outlined in Sections 3.5.1 and 3.5.2. Conceptually, if the beam is known to experience a certain stimulus field, increasing the end-load by some percentage should trigger remodelling assuming the change in stimulus level exceeds the limits of the lazy zone. After an unknown number of analysis steps, the stimulus field should return to within a certain percentage (governed by the size of the lazy zone) of the original reference signal.

A cantilevered beam $200 \mathrm{~mm}$ in length with a square uniform $30 \mathrm{~mm} \times 30$ $\mathrm{mm}$ cross-section was generated in Abaqus CAE. It was modelled using 20320 
C3D10M elements. The quadratic tetrahedral elements were used in order to validate the routine with the actual elements used in constructing the femoral model. Additionally, the mesh was more refined than required to accurately represent the geometry and loading of the beam. It was determined that if the element size is large relative to the stimulus field distribution within the part, stress concentrations were more likely to occur between adjacent elements. In other words, a change in element density had a greater influence when the element was large. If two adjacent elements had significantly different densities, the abrupt change in material stiffness would cause local stress concentrations. A similar problem occurred when elements of drastically different sizes were placed adjacent to one another. Consequently, the remodelling routine functions best with a fine, uniform mesh.

For the baseline case, the beam was assigned a uniform "bone density" of $1.0 \mathrm{~g} / \mathrm{cc}$. All nodes of the model were entirely restrained at the the fixed end, and two nodal point loads of $100 \mathrm{~N}$ each were applied at the upper corners of the free end. For the remodelling analysis, these loads were increased by $20 \%$, to $120 \mathrm{~N}$ each. The lazy zone was set as $\pm 5 \%$ and the remodelling rate constant was assigned a value of $50 \times 10^{-6}$. Density values were limited between 0.01 and $2.0 \mathrm{~g} /$ cc with step change in density limited to $\pm 0.10 \mathrm{~g} / \mathrm{cc}$. After 9 steps of remodelling, the stimulus field had been driven back to within the lazy zone and remodelling no longer occurred. The routine performed as expected, demonstrating an increase in density across all elements away from the neutral 
axis with the greatest increase $(1.0 \mathrm{~g} / \mathrm{cc}$ to $1.8 \mathrm{~g} / \mathrm{cc})$ occurring at the fixed end of the cantilever beam. Appendix $\mathrm{C}$ contains a summary of validation results.

\section{6 - Model Validation}

As a final step in preparing the resurfaced femoral finite element model for analysis, the intact femur model and the cadaveric femur specimen were compared to validate the model geometry and density calibration. Although care was taken when constructing the geometric model, and density calibration was performed through use of the CT calibration phantom, further calibration of the model may be required to ensure that strains obtained from the analysis of the resurfaced femur model are valid. Therefore the quality of the intact $3 \mathrm{D}$ femur model was verified by comparing strain readings from a mechanical test of the cadaveric femur to those obtained from a static finite element analysis of the intact femur with identical loading and boundary conditions.

\subsection{1 - Mechanical Test}

The mechanical test involved a single compressive load directed along the femoral shaft applied at the proximal region of the femoral head (Figure 2.12). The distal region of the femoral shaft was potted in bismuth. This region of the femur was considered to be completely rigid. The femur specimen was cleaned of soft tissues using a medical scalpel. Four TML Ltd. general purpose stacked

strain gauge rosettes (Appendix D) were fixed to the cleaned bony surface of the 
femur using general purpose superglue in the locations highlighted in Figure 2.13.

Data were collected using two Vishay Instruments strain gauge indicator and switch and balance units (Appendix D). The prepared femur fixture was aligned

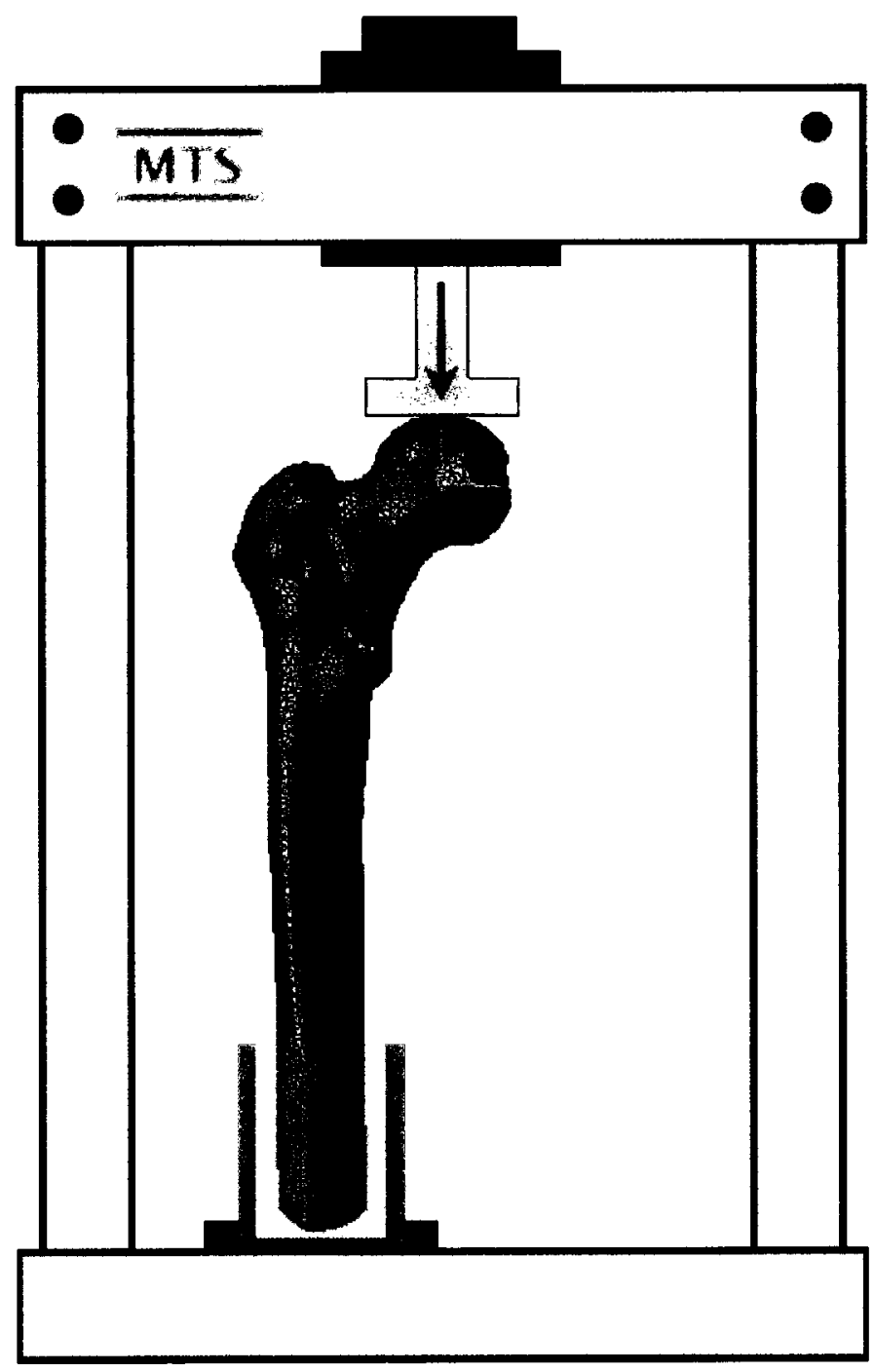

Figure 2.12: Specimen loading schematic. The test set up shown was used to validate the FE model against the cadaveric femur specimen. The specimen was loaded to $2000 \mathrm{~N}$ along the direction of the femoral shaft, which was parallel to the $z$ direction of the intact femur model. The distal region of the cadaveric femur was fixed in bismuth and fastened to the tray of the MTS load frame. The same region was constrained in the FE analysis. 


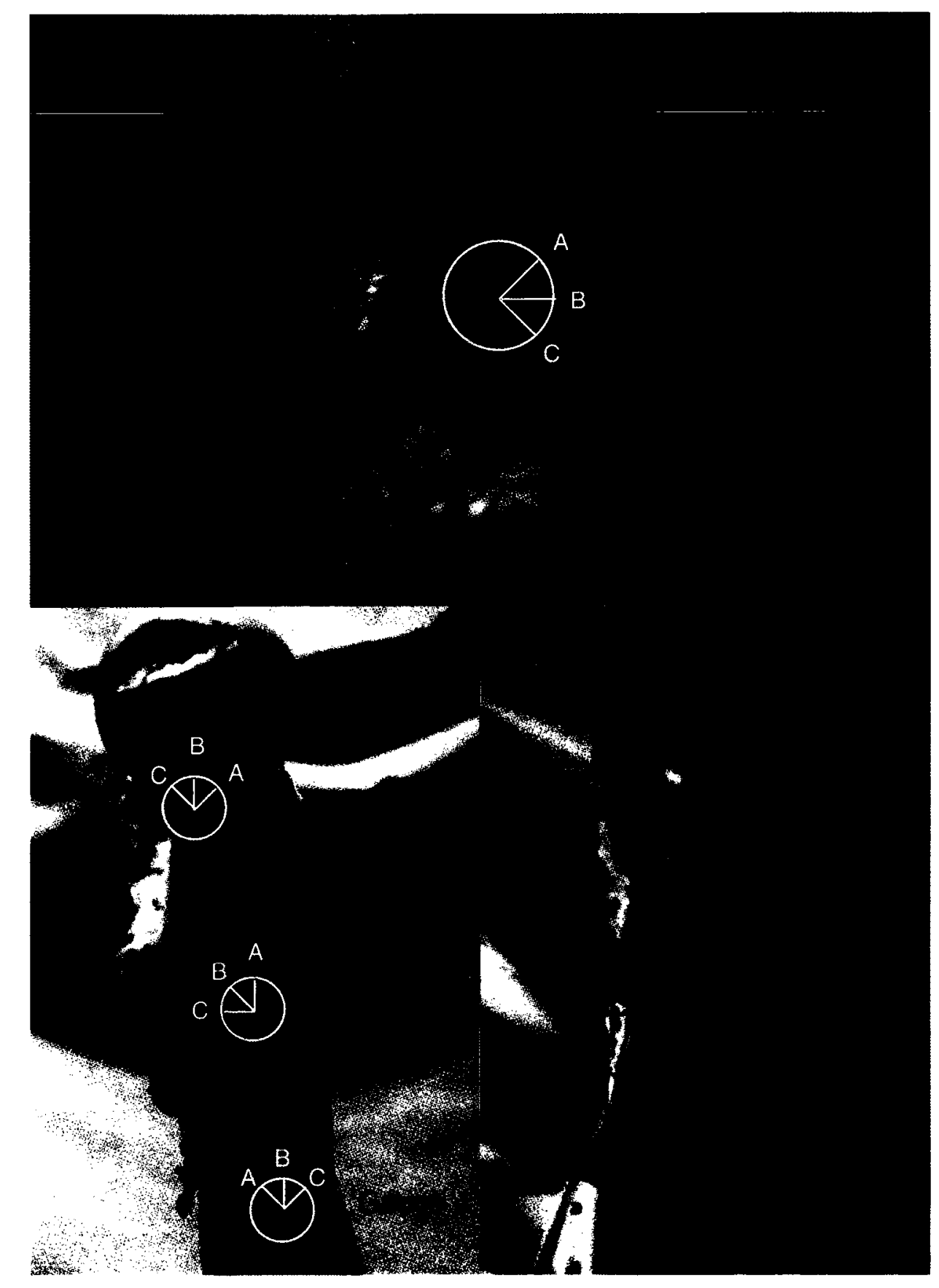

Figure 2.13: Cadaveric femur outfitted with strain gauge rosettes. A total of four gauges were fixed to the cleaned boney surface: one along the proximal region of the femoral neck between the greater trochanter and the femoral head (gauge 1, top), one on the femoral neck below the head (gauge 2, bottom left) and two along the femoral shaft (gauges 3 and 4, bottom left). The femur specimen was fixed in bismuth in preparation for mechanical loading (bottom right). 
in an MTS® actuated load frame for unidirectional compressive loading. The load was increased gradually from $0 \mathrm{~N}$ to $2000 \mathrm{~N}$, with strain readings taken at $1500 \mathrm{~N}, 1750 \mathrm{~N}$, and $2000 \mathrm{~N}$. The final deflection at the top of the femoral head was also recorded through displacement of the force actuator. After all data were collected, the load was removed and the process was repeated in its entirety (Appendix E).

During the first set of load increments, gauge 1 returned unreasonable readings between the $1750 \mathrm{~N}$ and $2000 \mathrm{~N}$ loads. After removing the final load, the gauge was inspected. It was observed that a significant amount of fluid had been released from the femoral head during compression. Some of the fluid had collected under and around gauge 1, and rendered this validation site unusable. It was therefore ignored during the second loading set.

\subsection{2 - Abaqus Analysis}

An analysis run was set up in Abaqus 6.8 to mimic the conditions of the mechanical test outlined in Section 2.6.1. The intact femur model was fixed entirely along the distal region corresponding to the potted area of the femur (Figure 2.13, bottom right). A set of direct loads were applied at nodes within a circular region on the proximal femoral head. The loaded region was determined using the deformed circular area observed when the load actuator was retracted from the specimen. 


\subsection{3 - Comparison}

In order to compare the data obtained during the mechanical test to the results of the Abaqus analysis, three element regions of the model corresponding to the attachment points of the strain rosettes were selected (Figure 2.14). The strain data collected from the test set-up was transformed into principal values using Mohr's strain circle theory [83]. Although the specimen experiences strain in all three principal directions, the gauge rosettes are limited to measuring strain in a particular plane. Consequently, the corresponding strain data requested from Abaqus were in-plane with an exposed element surface. The integration point values from elements 6349,10906 , and 12047 were averaged and selected to represent the locations of gauges 2,3 and 4 respectively. The elements were determined based on image landmarking. For example, the orientation of the femur model was matched to that of the photograph in Figure 2.13 (bottom left) by identifying 3 landmarks: the fovea of the head of the femur, the lesser trochanter, and the visible region of the greater trochanter. Gauge positions were triangulated from landmarks and their approximate locations were transferred from the photographic image to the model. The method applied was repeatable within $3 \mathrm{~mm}$ of the originally determined gauge positions. This corresponds to the side length of one element. Element strains and corresponding measured strains are summarized in Table 2.6. Note that the direction of the first principal strain is measured relative to the direction of the femoral shaft. The global zdirection in the Abaqus model is parallel to the longitudinal direction of the 


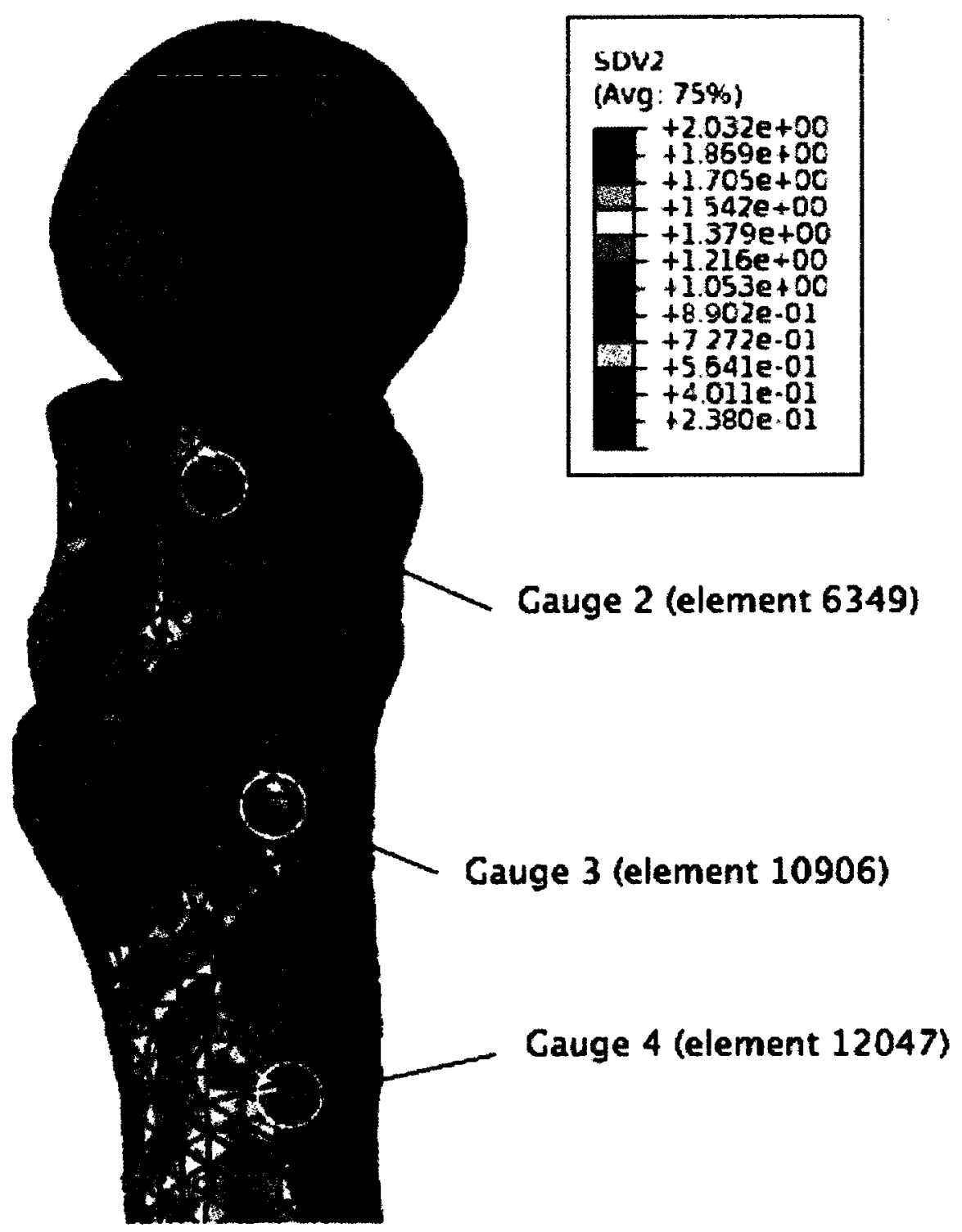

Figure 2.14: Density field distribution within the intact femur model. The gauge locations and their corresponding elements are highlighted. The rosette matrix material is circular and approximately $5 \mathrm{~mm}$ in diameter (not to scale); however, the rosette is composed of 3 stacked strain gauges $1 \mathrm{~mm}$ in length, thus the area taken up by the actual gauge stack is significantly smaller than the rosettes matrix $(\sim 1.5 \mathrm{~mm}$ across). The strain readings were taken at a location centered in the white circles. Note the density hotspot adjacent to gauge 2. All density values are in $(g / c c)$. 


\begin{tabular}{|c|c|c|c|c|c|}
\hline & & \multicolumn{2}{|c|}{ Cadaveric Femur } & \multicolumn{2}{|c|}{ FEM } \\
\hline & & Set $\mathbf{A}$ & Set $\mathbf{B}$ & In-plane & Max Comp. \\
\hline Gauge & Value & \multicolumn{4}{|c|}{ Microstrain } \\
\hline \multirow{3}{*}{$\begin{array}{c}2 \\
\text { (Element } \\
6349)\end{array}$} & $\varepsilon_{1}$ & 1223.7 & 942.4 & 883 & \\
\hline & $\varepsilon_{2}$ & -2786 & -2528 & -1578 & -2509 \\
\hline & $\Theta$ & $-3.4^{\circ}$ & $-2.8^{\circ}$ & $7.2^{\circ}$ & \\
\hline \multirow{3}{*}{$\begin{array}{c}3 \\
\text { (Element } \\
10906)\end{array}$} & $\varepsilon_{1}$ & 332.1 & 339.8 & 559 & \\
\hline & $\varepsilon_{2}$ & -3360 & -3616 & -3362 & -3702 \\
\hline & $\Theta$ & $3.8^{\circ}$ & $4.1^{\circ}$ & $6.4^{\circ}$ & \\
\hline \multirow{3}{*}{$\begin{array}{c}4 \\
\text { (Element } \\
12047)\end{array}$} & $\varepsilon_{1}$ & 556 & 540 & 636 & \\
\hline & $\varepsilon_{2}$ & -2416 & -2392 & -3017 & -3211 \\
\hline & $\Theta$ & $5.3^{\circ}$ & $5.3^{\circ}$ & $6.8^{\circ}$ & \\
\hline
\end{tabular}

Table 2.6: Strain reading comparison between cadaveric femur and FEM results. Principal strain directions, $\theta$, are measured relative to the longitudinal femoral shaft direction. Positive angles are clockwise relative to the strain gauge orientation. The Max Comp. column is the fully $3 D$ compressive strain value for the element, in the global model coordinate system.

shaft. Each rosette contained one gauge which was directed along the shaft. The angle was listed relative to this gauge (Figure 2.13 illustrates strain gauge orientations). The "Max Comp." strain is the fully 3D maximum compressive strain recorded in the listed element taken in the global coordinate system. This strain was not necessarily in-plane with the element face used to represent the strain gauge locations; thus, it was out of plane with the actual gauge readings. However, because of the nature of the mechanical test setup, it was reasonable to expect that the maximum compressive strain would occur almost in-line with the 
direction of the load (causing both longitudinal compression and bending strains in the shaft), and would therefore be nearly in-plane with the open surface of the element.

The readings from gauges 3 and 4 matched well with those of the finite element model, especially considering the nature of the test specimen. The magnitudes of the principal strains determined from the finite element model were within 200 to 600 microstrain of the recorded values (7-26\%) and their directions are nearly aligned. The in-plane values returned at element 6349 (gauge 2), although within a similar range, were less in agreement with the rosette readings than the other two gauges. Values from the second principal strain returned from the FE model were different by up to 1000 microstrain. This may be attributed to one or both of the following reasons: the gauge orientation on the element face is out of plane or the density "hotspot" adjacent to this gauge (Figure 2.14) caused lower model strains than expected.

The strain gauge rosettes recorded strains that are in-plane with the surface of the femur. In the case of the FE model, this translated to strains inplane with the "exposed" surface of the elements. If the landmarking process resulted in a misalignment, the curvature of the femoral neck at this point can cause offset in the magnitudes of the in-plane strains. Querying an element directly to the right of element 6349 , as per the orientation of Figure 2.14, returned a maximum in-plane compressive strain of -1900 microstrain. This is closer to the values listed for gauge 2 in Table 2.6 than the in-plane strain from 
element 6349 . Moving one more element to the right of 6349 returned an even closer value of -2401 microstrain. The maximum compressive strain in full $3 \mathrm{D}$ remained around -2500 microstrain while taking queries one and two elements to the right of element 6349. As mentioned, the landmarking process was repeated within $\pm 3 \mathrm{~mm}$, which translates to the edge length of one element. Therefore, it may be unreasonable to excuse the discrepancy entirely as an error in alignment. The elements selected (Table 2.6) were found to be the most likely locations of gauges 2-4.

The discrepancy between gauge 2 and its corresponding element may also be attributed to the large variation in density present near gauge 2 (Figure 2.14b) where the density varies from $-1.0 \mathrm{~g} / \mathrm{cc}$ to $-2.0 \mathrm{~g} / \mathrm{cc}$ across only 4 elements. It is possible that large density values $(>1.8 \mathrm{~g} / \mathrm{cc})$ are not accurately calibrated from Equation 1.2. Because the compressive strain was lower in the model than in the mechanical test, this indicates that the higher densities in the FE model may have been assigned and elastic modulus larger than is actually present in the specimen. Equation 1.2 is an exponential relationship of the form $A \rho^{\mathrm{B}}$; thus, it is possible that in the lower density ranges $(<1.5$, near gauges 3 and 4$)$ the calibration is reasonably accurate but it loses validity for larger densities. Parameter identification and validation of the density-elastic modulus relationship is beyond the scope of this thesis.

Despite the disagreement of gauge 2 relative to its corresponding element, the overall correlation between results of the finite element model and the 
mechanical test was excellent considering that bone is an anisotropic, heterogeneous biological material. It is important to note that there was no clear calibration factor that could be applied to the current intensity-to-density function to bring the calculated strains closer to the measurements. Specifically, because principal strains in the FE model were both larger (gauge 4, ع2) and smaller (gauge $3, \varepsilon 2$ ) than those measured from the mechanical test, it was therefore impossible to apply a simple linear calibration factor to the density. Insufficient data were collected for accurate non-linear calibration. Based on the good overall match of strain measurement and the FEM results, the model was considered to be valid.

\section{7 - Implementation}

After the intact and resurfaced FE models were generated and validated the desired test cases were implemented. Three simplified bone-implant interface conditions (Figure 2.15) were investigated: i) complete fixation (tied constraint across all interface surfaces), ii) friction contact across all surfaces, and iii) a hybrid of the first two cases where the implant peg is modelled with friction and the inner cap-like surface of the implant is tied. Each set of interaction properties was expected to provide insight into the nature of stress shielding caused by the cementless femoral resurfacing implant. 


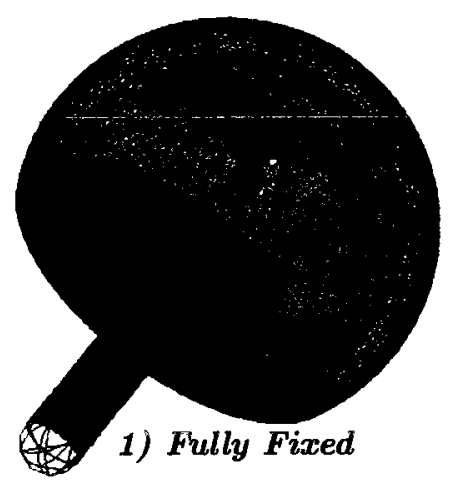

Fixed Surface
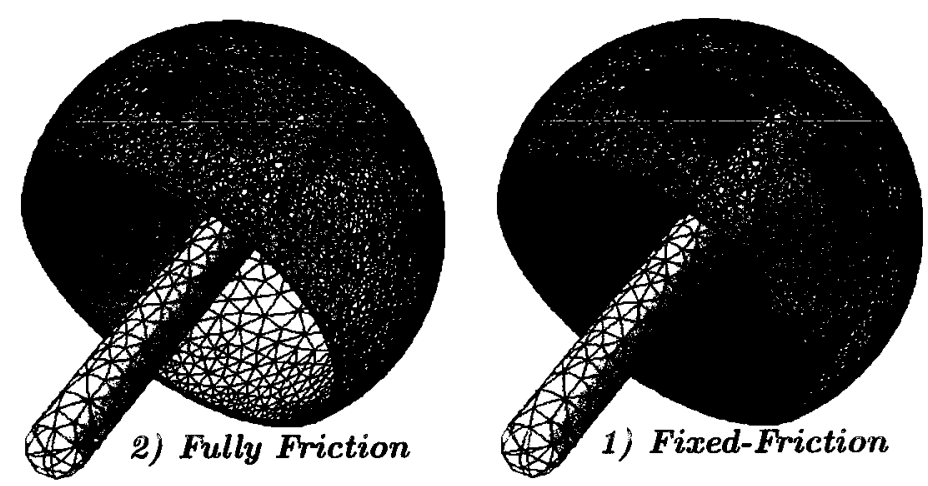

Friction Surface

Figure 2.15: Three implant interface models. Case 1) involves fixing the entire inner surface of the femoral cap as well as the peg. Case 2) models all inner surfaces as friction interfaces. The final case, 3), is a hybrid model where the inner surface of the cap is tied, but the peg is modelled with friction.

\subsection{1 - Baseline Analysis}

The concept of the baseline analysis run was introduced in Section 2.5.1. Bone adaptation relies on a reference signal: a stimulus field that the tissue would feel under normal anatomical circumstances. The baseline analysis was necessary to establish the expected stimulus field. The loading and boundary conditions used to define the analysis must be propagated to any subsequent remodelling tests. Any changes in the loading conditions would require preparing a new baseline analysis to obtain the corresponding reference stimulus field.

For the baseline analysis, the intact femur model was fixed around the circumference of the femoral shaft just distal of the lesser trochanter. Radiological studies performed by Taylor et al. [84] show that the femur 
experiences primarily compressive forces without significant bending. Ideally, applying a full set of muscle loads to a femoral model should reproduce the results seen in the radiological study; however, to do so would require a complete femur model. The specimen in this study was severed above the femoral condyles (knee joint). Because the most severe effects of the resurfacing implants on stress shielding are known through clinical observations to remain local to the femoral head and neck, the model was fixed just distal of the lesser trochanter (Figure 2.16). A more distal location would increase the bending effects caused by the head load and artificially increase the maximum stresses seen in these regions of the model. The lesser trochanter was sufficiently distal to the implant and

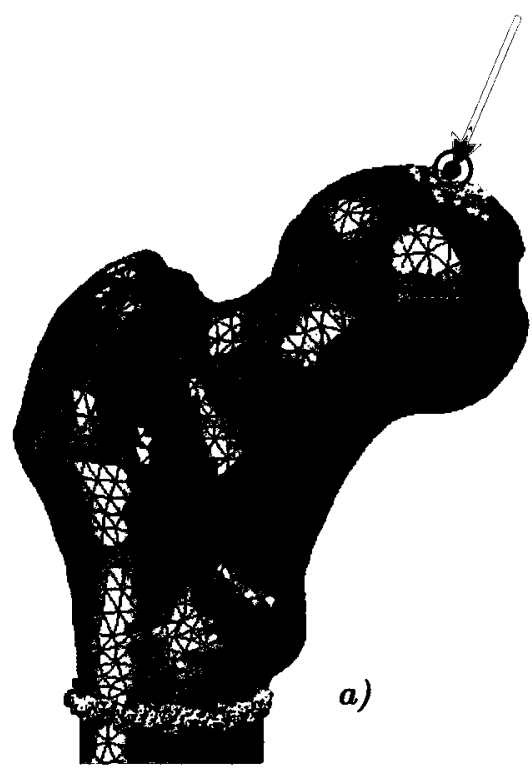

$$
\text { Inferior-lateral load }
$$

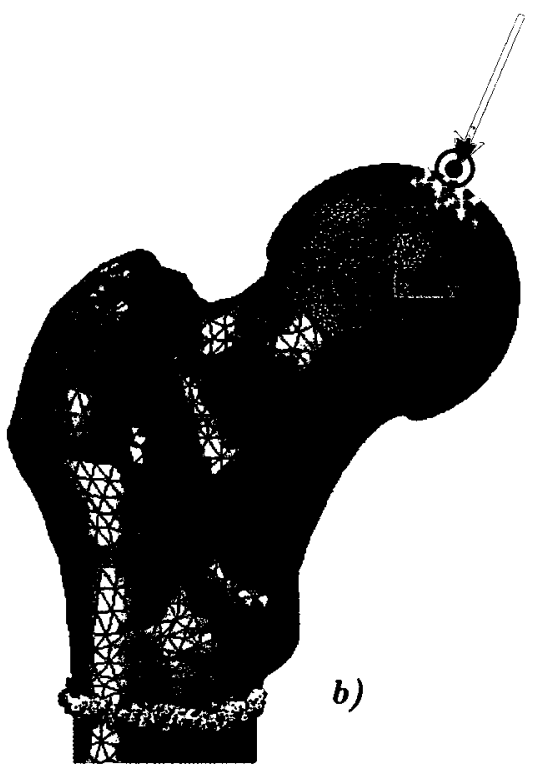

- Posterior load

Figure 2.16: Femur model loading and boundary conditions. The boundary conditions from the intact femur model (a) are transferred directly to the resurfaced model (b). The resurfaced model experiences the same load magnitude as the intact model. In both models the load is shared evenly over 12 nodes and directed through the head-center. 
femoral neck to minimize the bending effects and avoid any significant influence of the boundary condition on the simulation of the proximal femur.

The femoral head was loaded with three load components, as determined by Bergmann et al. [36] (Section 1.2.3.1). The load applied was taken from the peak walking data and patient weight was assumed to be $80 \mathrm{~kg}$. More specifically, the three force components were determined using $225 \% \mathrm{BW}, 54 \% \mathrm{BW}$, and $30 \%$ BW in the inferior, lateral, and posterior directions (Figure 2.16) which returns a load magnitude of $1831 \mathrm{~N}$. In Abaqus, a load-specific coordinate system was located at the center of the femoral head with the $x-y-z$ directions corresponding to the aforementioned anatomical directions respectively. The load was directed through the head-center and applied evenly over 12 nodes on the surface of the femoral head to minimize the effects of the concentrated load. The nodes were distributed over a circular area with a projected diameter of approximately $16 \mathrm{~mm}$ so as to simulate an area of application similar to the one observed during the mechanical test. The baseline Fortran subroutine was used to import density data and assign element properties through the elastic modulus field variable. Figure 2.16a illustrates the model for the baseline analysis in Abaqus.

\subsection{2 - Remodelling Analysis}

The final product of the development phase of this thesis was an adaptive finite element model capable of varying the density, and consequently the elastic modulus of individual elements in order to simulate the process of bone 
remodelling. The resurfaced femur model was fixed and loaded in an identical manner to the intact femur used in the baseline analysis (Figure 2.16b). In the case of the fixed boundary condition, the node set used to defined this condition was transfered directly from the intact model to the resurfaced one. This was possible because of the identical mesh definitions used in reconstructing the intact and resurfaced models from the reamed femur (Section 2.3.2). The head load could not be transfered directly. The load was distributed over nodes on the intact model that were "reamed away" in the resurfaced model. The area defined by the 12 nodes selected in the baseline analysis was transferred to the resurfaced model and 12 nodes from the implant mesh were selected within this area. The magnitude and direction of the load (1831.14 $\mathrm{N}$ through the head-center) was identical to that of the baseline case.

Two surface pairs were identified on the model in order to define the interaction between the implant and the bone: a cylindrical region around the outside of the peg and a bowl shaped region on the inside of the implant (Figure 2.17). These surfaces were on the reamed femur geometry. From these two surface pairs, three contact conditions were established. The first case, the simplest, involved fixing both bowl and peg to their corresponding femur surfaces. This case idealized the situation where the entire implant was coated or surface treated to encourage bone integration on the metallic surface and sufficient recovery time has been permitted to allow complete in-growth. There was essentially no motion between bone and implant and there was no buffer zone 


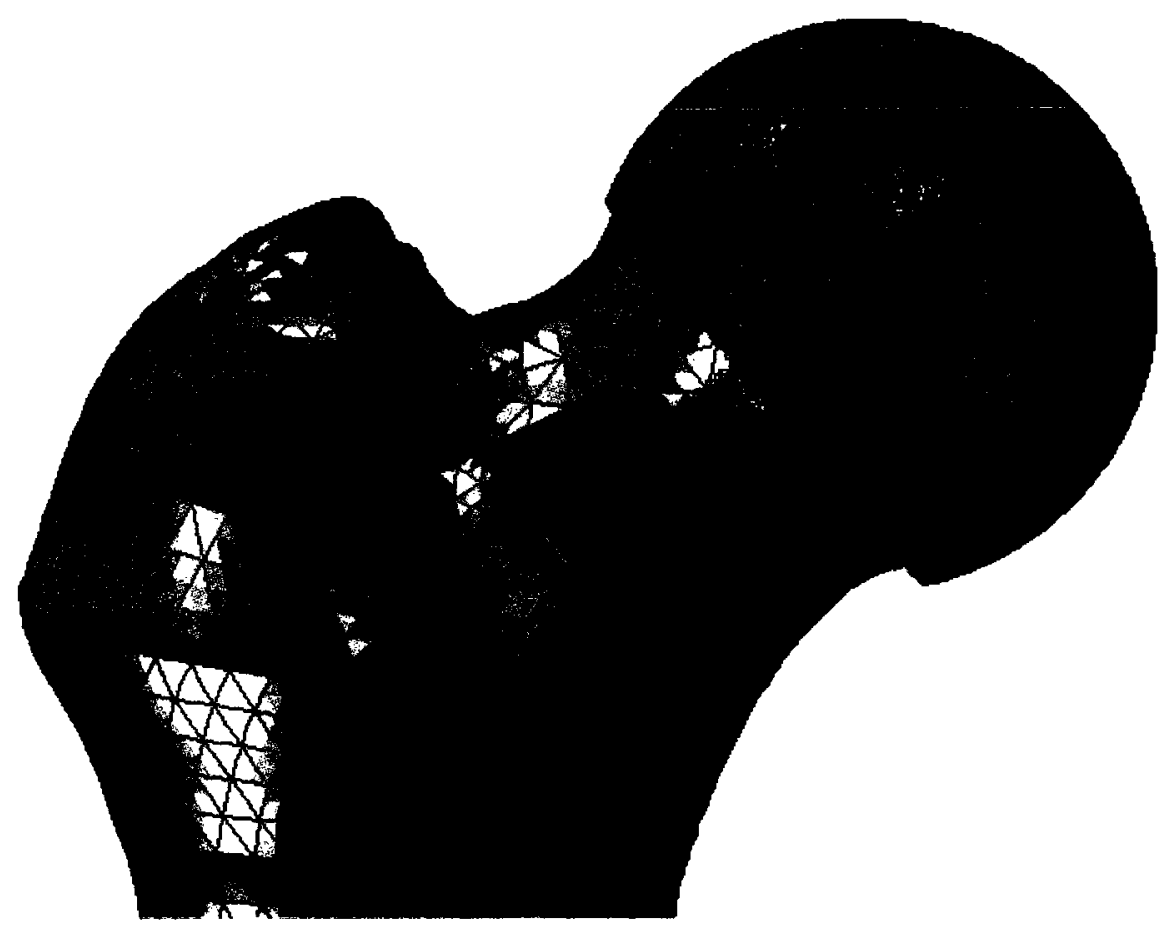

Figure 2.17: Surfaces used for implant-femur interaction. The slave surfaces, the cap and peg, were both from the implant mesh. There is no overlap between the contact pairs. The peg surface does not extend to the semi-spherical tip of the implant to avoid causing element collapse in this region of the femur model.

that would be provided by bone cement in cemented hip resurfacing arthroplasty. In Abaqus, this restriction was modelled by a tied constraint between the two surface contact pairs: a) the implant cap to the corresponding reamed surface, and b) the peg to the surface defining the reamed hole. A surface-to-surface formulation was selected to avoid stress noise at the interface; however, the increased complexity of this approach may have added to analysis run-time. In both contact pairs, the master surface was selected to be on the reamed femur part. The slave nodes on the implant were adjusted to be initially in contact with the master surface to ensure complete fixation. 
The two other cases both make use of friction interactions in Abaqus. Press-fit interference results published by Gebert et al. [85] were used to estimate the tangential coefficient of friction in the primary stability of a press-fit cementless resurfacing implant (without in-growth on the implant surface). In essence, the friction contact was used to model the case where a cementless implant was not fully integrated with the adjacent bone tissue. A static friction coefficient of 0.4 (identified as "medium bone quality" by Gebert et al., and the mode of their test samples) was used to control the tangential friction interaction. The same master-slave surface pairs used for the tied constraint condition were used for these cases. Once again, surface-to-surface contact was selected to ensure accurate stress results. Because large sliding motion between the implant and bone surfaces would essentially indicate failure of the implant, and should not occur in the analysis, the interaction was governed by Abaqus' small-sliding formulation [76]. The tangential friction was assumed isotropic and no critical stress limit was defined. Normal contact stiffness was modelled using linear behaviour. Both the tangential and normal interaction properties were enforced using the penalty function method.

Two cases were analyzed using this friction interaction property. The first assumed that none of the implant surfaces were integrated with the bone; therefore, all interfaces were modelled using friction contact. The last analysis case involved both friction and a tied constraint. Specifically, the cap surface of the implant was tied to its corresponding surface on the femur model while the 
peg surface pair is assumed to be press-fitted and is modelled with friction contact (Figure 2.15). This case best resembles the clinical problem where a cementless implant may have been allowed to fully integrate with the bone tissue (assuming ideal and complete in-growth) on the inner surface of the implant but the peg was used purely for alignment purposes. In both cases, slave nodes on the implant surface were adjusted to be in initial contact with the bone material. 


\section{Chapter 3}

\section{Results}

The results of the 3 simulations, namely, completely fixed, friction, and fixed-friction, outlined in Section 2.7.2, gave insight into the nature of stress shielding present in the uncemented resurfaced femoral head. All the state variables stored within the subroutine during the Abaqus analysis were available as an output field. The analysis of the output fields, such as the bone density, was performed through qualitative and quantitative comparison of the changes induced in the remodelling stimulus and bone density by each type of fixation. The model was cross-sectioned and subdivided into 7 subsections, as shown in Figure 3.1, for the purpose of analysis and discussion.

\section{1 - The Implant's Effect on the Stimulus Field}

Bone remodelling is motivated by a change in the stimulus field relative to the baseline value. Recall that the stimulus for the purpose of this thesis was the ratio of strain energy density and apparent bone density (Equation 1.3). Because the presence of the implant initially had no effect on the local bone density, the initial change in stimulus was governed by changes in the stress/strain field. Before any remodelling took place, it was possible to estimate the inevitable changes in bone density by comparing the stimulus fields in the different resurfaced femur cases to that of the intact femur. 


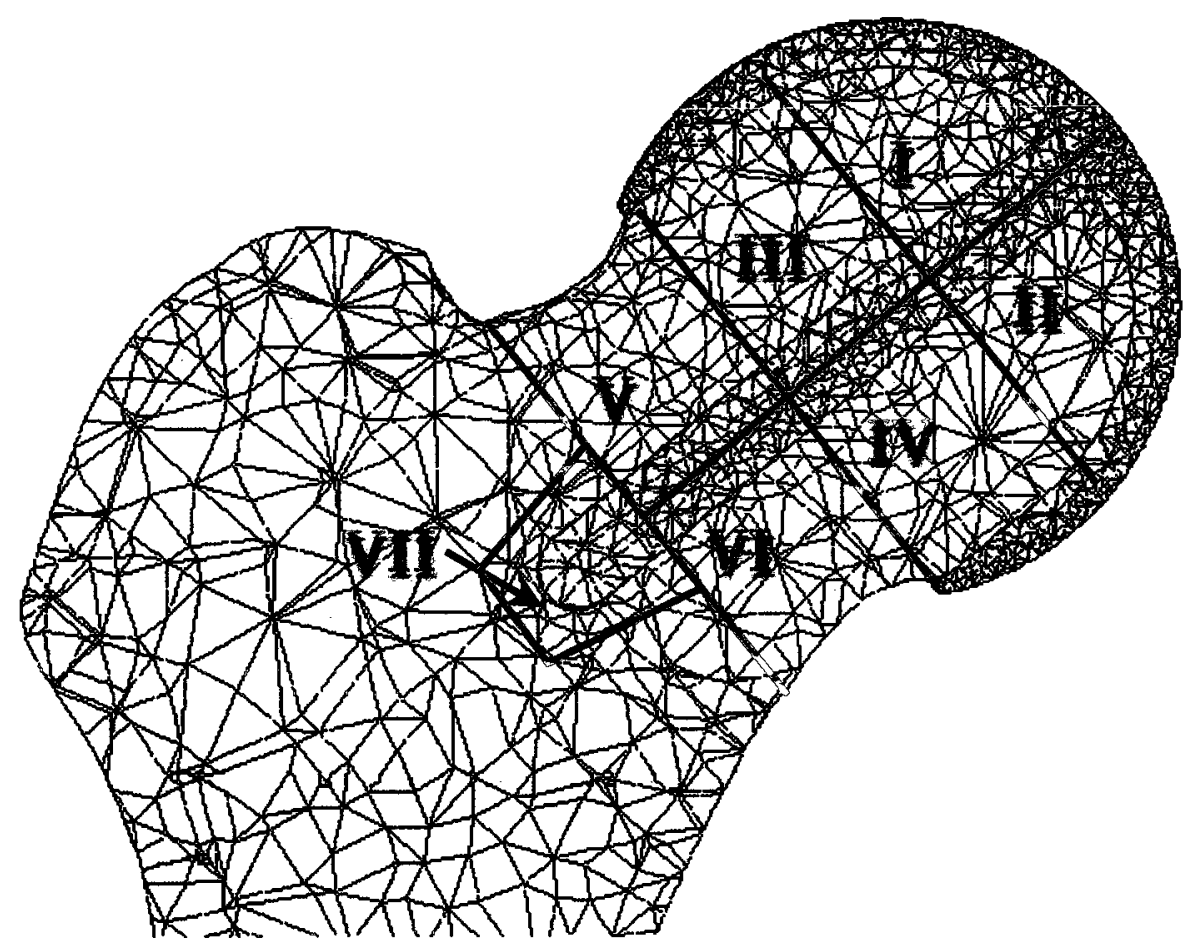

Figure 3.1: Enumerated regions of the resurfaced femoral model. Regions I through IV are located in the femoral head, regions $V$ and VI in the neck, and region VII surrounds the tip of the implant peg.

Figure 3.2 shows the baseline stimulus levels throughout the regions of interest within the femoral head and neck in comparison to the initial stimulus levels of the three different implant contact cases taken at step 2, before remodelling occurs. Recall Figure 2.15, which outlines the three contact cases. Both cases where the cap surface was fixed to the bone material resulted in a significant drop in stimulus within regions I and II, with large drops in regions III and IV as well. This was expected to produce a large degree of bone resorption in this region. The friction model, case 2, also showed a drop in stimulus in regions I and II regions; however, region II (below the implant peg) demonstrated 

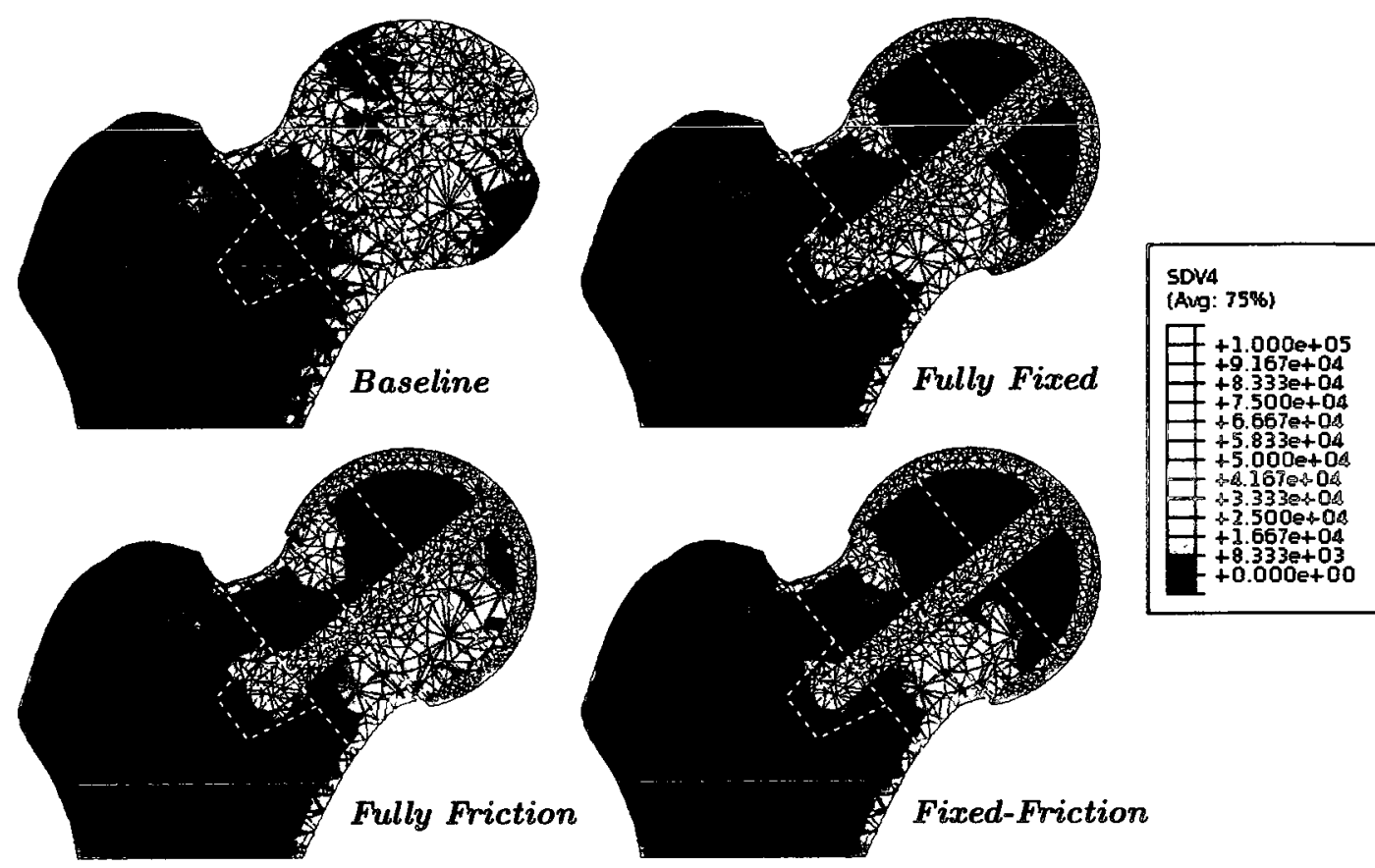

Figrere 3.2: Initial remodelling stimulus for each implant case. The intact femur stimulus field (buseline) is used as the reference state. Changes in this field will induce remodelling in the local bone. The stimulus field variables is output at the beginning of analysis step 2 (before any bone remodelling). The fully fixed case shows significant variation in the stimulus field, particularly in the proximal femoral head (regions I through IV). The same is true for the fixed-friction case, where the cap surface was tied and the peg was modelled with friction. The case of entirely frictional contact shows less of a change in regions III and IV, though still a significant drop in I and II. All stimulus values are in $\mathrm{J} / \mathrm{g}$.

less change than in the fully fixed or the fixed-friction case. Also in the fully friction case, regions III and IV showed very little variation from the baseline stimulus. The two cases involving the fixed cap surface also showed a significant decrease in stimulus level between the peg and the upper cortex of the femoral neck (region V). Finally, all three cases demonstrated an increase in stimulus around the distal tip of the peg, in region VII. This region is unaccustomed to carrying large loads. 


\section{2 - Convergence of the Remodelling Routine}

The analysis of each case was completed after allowing the process of remodelling to occur until there were no step-wise changes in density (after 8-10 analysis steps). In the case of these analyses, this may indicate that the stimulus field throughout the model had settled within the lazy zone of the remodelling algorithm, or that further changes in local density were impossible on account of the density range limitations. In all three simulations, most of the remodelling had occurred by the 7 th or 8 th analysis steps, with subsequent steps demonstrating little to no changes in local bone density. Because the lowest possible density was limited to $0.01 \mathrm{~g} / \mathrm{cc}$, bone cannot be completely resorbed. In the case of the remodelling analysis, this means that some regions may be incapable of reaching the baseline stimulus level and cannot fall into the remodelling lazy zone. More specifically, if regions in the femoral head experienced little to no strain after hip resurfacing, the subroutine attempted to increase the stimulus level felt by these regions by decreasing the local bone density. In these cases, the density was driven to the minimum value and stagnated there. This prevents the analysis code from reaching remodelling equilibrium in the affected areas. In these situations, stability must be determined through observation of local density variation.

This was observed in the first and third analysis case with the fully fixed implant and the fixed-friction implant (Figure 3.3). In these cases with a tied implant cap, convergence in regions I and II proved impossible on account of the 

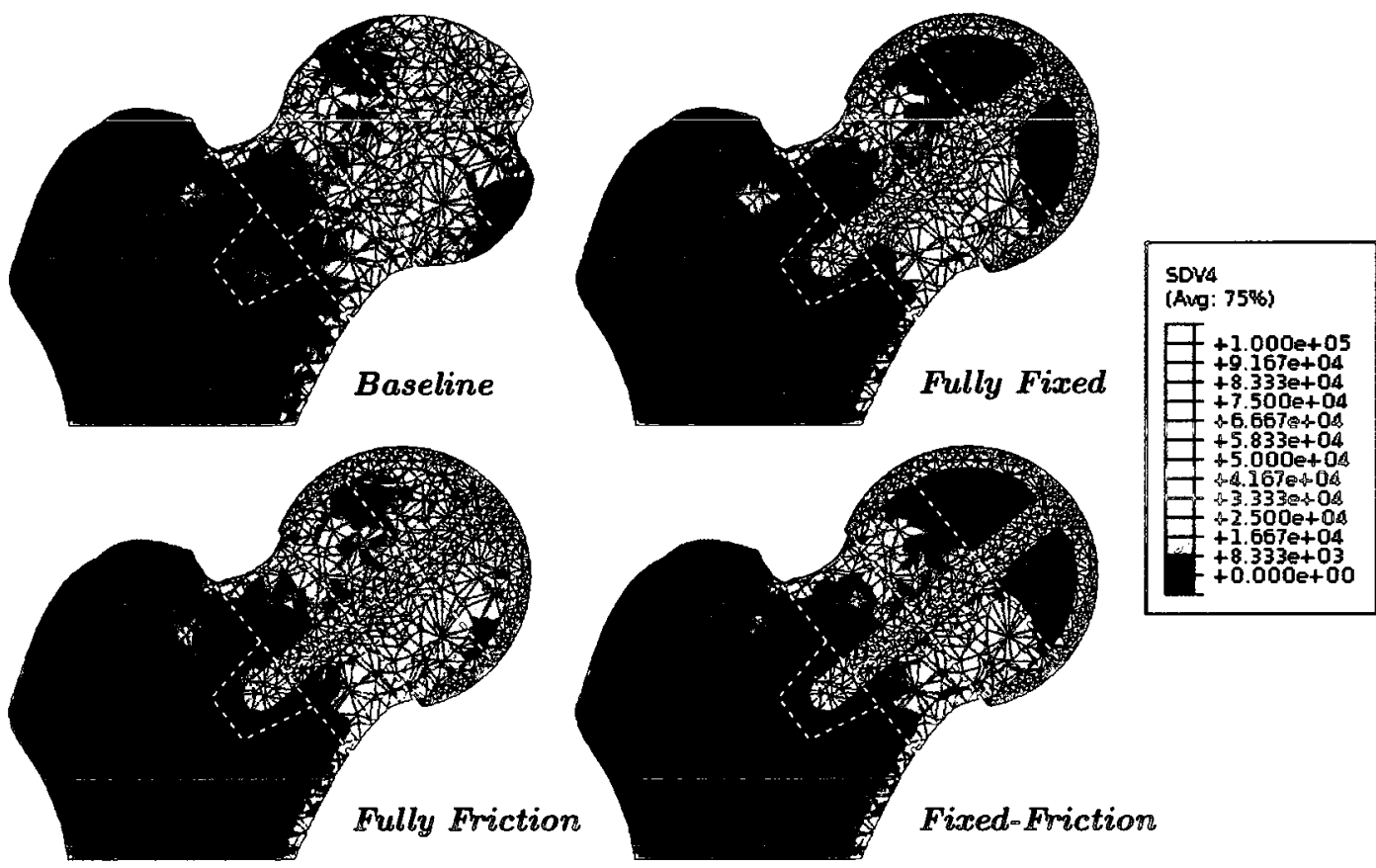

Figure 3. \$: Final remodelling stimulus for each implant case. The fully friction model was capable of returning to the reference stimulus state through remodelling of the bone density. Convergence within the lazy zone occurred by the 10th analysis step. Both cases with tied implant caps (fully fixed and fixed-friction) experienced a dead zone in the proximal femur (regions I and II). This zone is incapable of undergoing further remodelling, having been limited to a minimum density of $0.01 \mathrm{~g} / \mathrm{cc}$. Regions III through VII, near the neck and away from the top of the femoral head, resemble the baseline stimulus field. All stimulus values are in $J / g$.

minimum density limit. In the second case, where all interactions were modelled with friction (Figure 3.3), the analysis converged back to the original baseline stimulus across all regions of the model; the stimulus feedback signal returned within the lazy zone, which confirmed the reliability of the subroutine.

\section{3 - Final Density in the Resurfaced Femoral Head}

After remodelling has completed, whether it be because the stimulus field returned to within the lazy zone or because the density no longer showed 
significant change between steps, the final density distribution throughout the femoral head can be investigated. Figure 3.4 contrasts the initial density in the femoral head to the results presented by the three resurfacing scenarios. Not surprisingly, all three cases demonstrated significant bone density decrease in the proximal region of the femoral head. The pure friction model showed the least resorption of the three analyses. The average resorption across regions III and IV was limited to approximately $15-20 \%$. This estimate does not take into consideration the small local increases in density visible in these regions. The
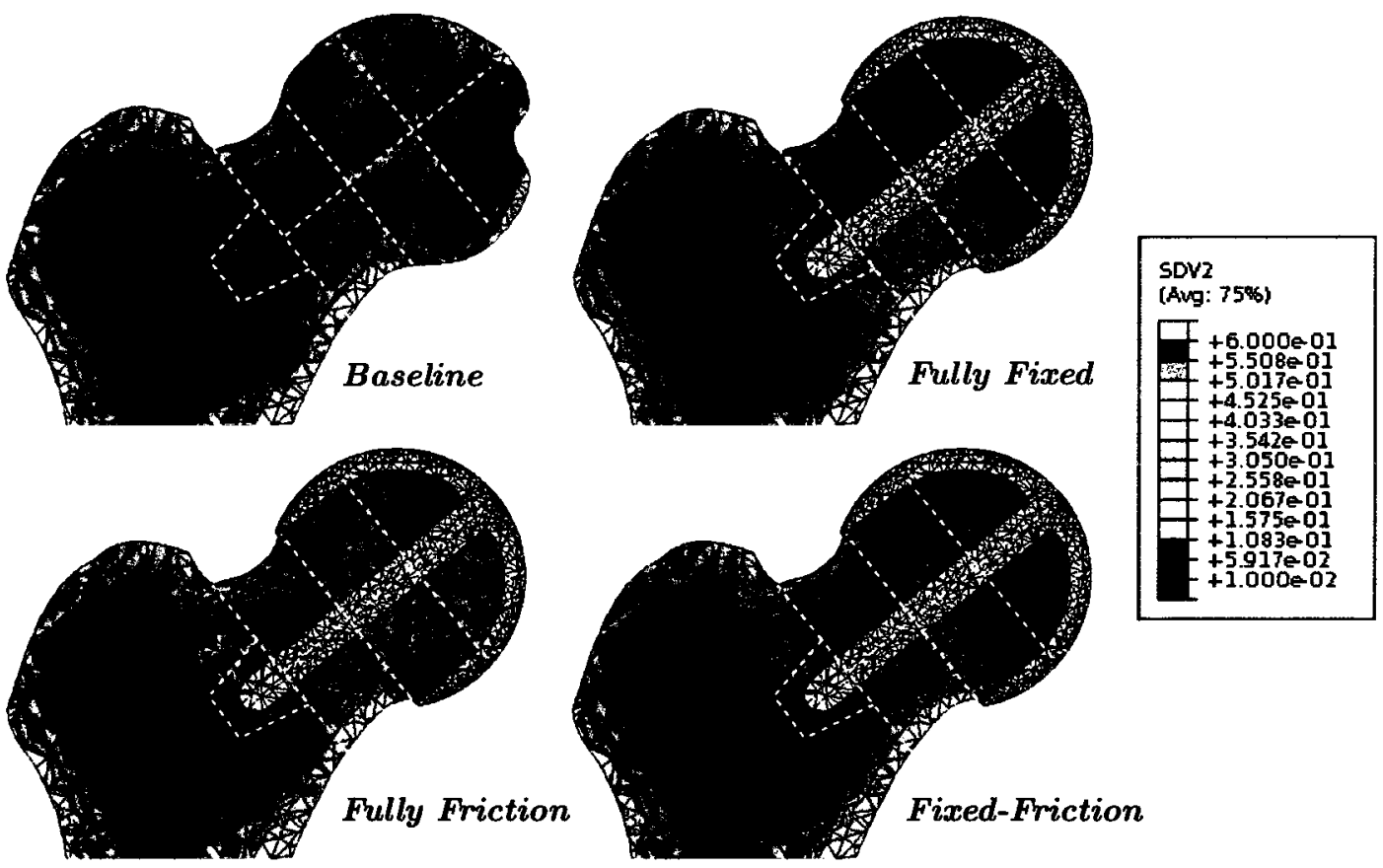

Figure 3.4: Final bone density distribution for each implant case. Both cases with a tied implant cap (fully fixed and fixed-friction) show significant resorption of the bone in the proximal femoral head. In all three cases the implant peg causes hypertrophy in bone tissue in region VII. This is most evident in the entirely friction based case. More resorption occurs in region $V$ in the fully fixed case than in pegs modelled with friction interaction. Regions in black are an artifact of Abaqus' extrapolation algorithm for displaying field variables in the output database. These regions have a density of 0.01 (min. allowable). No integration points can be assigned density values below 0.01. All density values are recorded in $\mathrm{g} / \mathrm{cc}$. 
pure friction model did, however, show noticeable resorption in the proximal femoral head around region I where integration points within this region showed between $60 \%$ and $90 \%$ resorption and stagnated at the lower density limit. There was a small region of hypertrophy (a hotspot) within region VII that showed a considerable increase in local density. There was also a narrow region adjacent to the upper lip of the implant near the boundary between regions I and II that experienced a localized bone loss exceeding $90 \%$. This small region also stagnated at the minimum density value.

Both the first and third implant cases, which included the tied cap surface, showed a much larger decrease in density within regions I through IV. Regions I and II showed decreases in density between 70 and $95 \%$, region III showed a decrease of approximately $80 \%$, and region IV showed decreases ranging from 40-60\% In general, regions II through IV showed significantly greater density loss in cases with a tied implant cap than in the fully friction case. These two conditions also exhibited the hypertrophy observed in region VII, as well as the narrow region of density loss at the boundary of regions I and III and a generic decrease in the density of the upper cortex in region $\mathrm{V}$. The region of hypertrophy at the tip of the peg is noticeably smaller in these cases relative to the fully friction case; however, the small region of bone loss near the implant lip was equally evident between the three. Tying the implant cap to the bone also introduces another hotspot at the boundary of regions IV and VI (Figure 3.4). 
When the peg surface was tied to the surrounding bone in the fully fixed case, a large decrease in bone density occurred between the upper surface of the implant peg and the cortex of the femoral neck, seen in region $\mathrm{V}$ of Figure 3.4. This feature was not evident in either of the other two cases. Conversely, more load was carried by the bone below the tied peg and thus an increase in bone density was observed between the peg and the lower cortex in region VI. A series of output results depicting the change in density during each step of the analysis can be found in Appendix F.

Focusing on the change in the cortex of the femoral neck, the most apparent trend was a general decrease in bone density ranging between 0.1 and $0.3 \mathrm{~g} / \mathrm{cc}$. This density reduction of the cortex may indicate neck narrowing. It is evident in all three scenarios but is slightly less apparent in the fully friction scenario. Figure 3.5 shows the trend of decreasing bone density in the cortical bone around the femoral neck. The figure is based on the absolute change in density; thus, regions like the cortex in region VI may experience large changes compared to those of the upper cortex of the neck simply because the bone in the lower cortex was more dense to begin with. Nonetheless, both upper and lower cortical regions showed a clear trend of decreasing bone density. Another interesting result as seen in Figure 3.5 is the pattern created by bone reduction in the femoral head. This represents the complete absorption of the natural trabecular structure (as present in the baseline image in Figure 3.4 or in the X- 

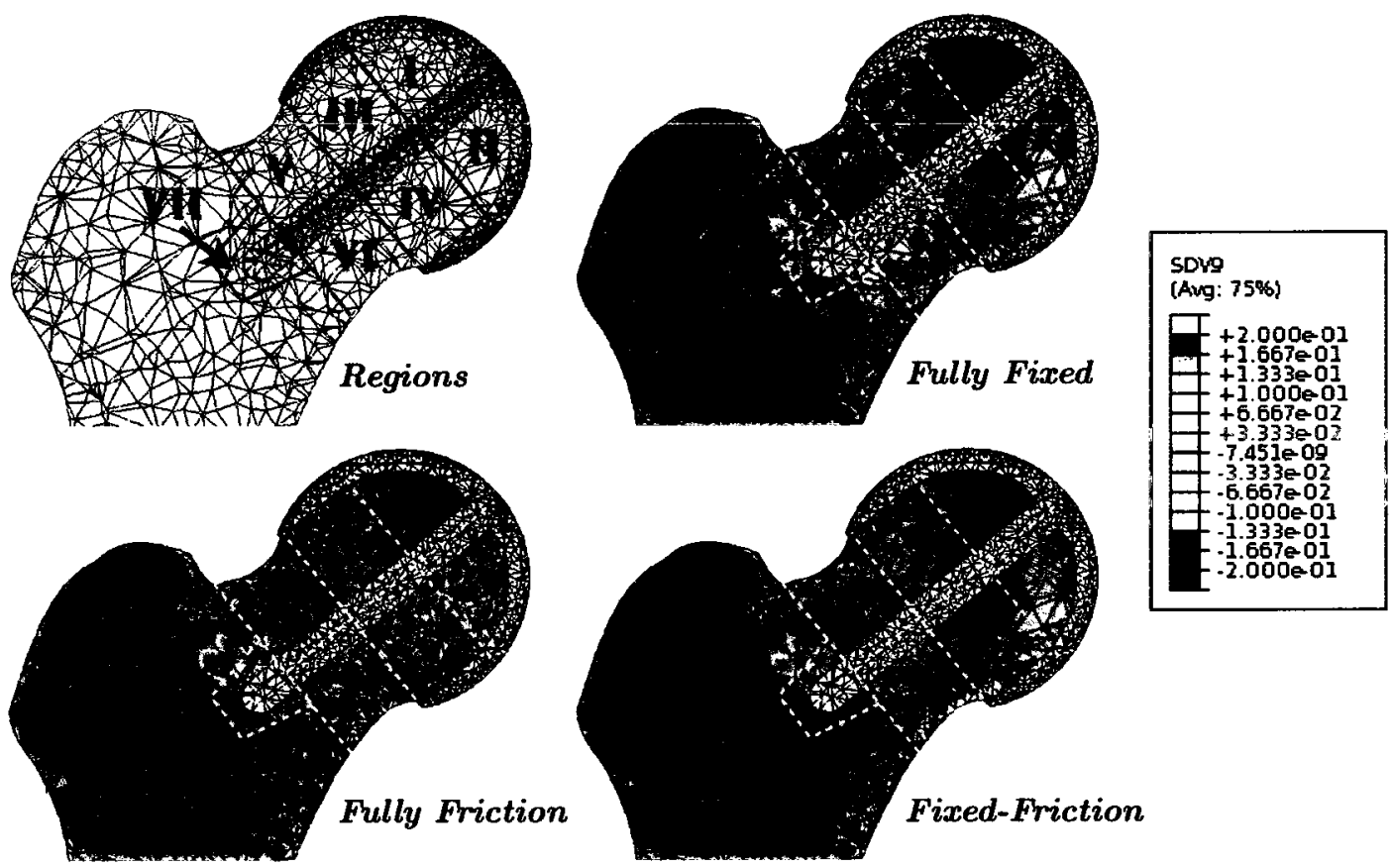

Figure 3.5: Absolute change in bone density for each implant case. Regions in black exhibited a decrease in bone density of more than 0.2. The blue and black regions along the cortex indicate narrowing of the cortical layer. This was observed in all three resurfacing models. All relative density values are in $\mathrm{g} / \mathrm{cc}$.

ray in Figure 1.3) that connects the loading point on the surface of the femoral head to the lower femoral cortex. 


\section{Chapter 4}

\section{Discussion}

It remains to analyze and discuss the results of the remodelling simulation. The subsequent sections consider the performance of the simulation in the study and the clinical implications of their results. In addition, the resulting density changes are compared to published studies involving hip resurfacing implants.

\section{1 - Performance of the Remodelling Analysis}

Both the theoretical remodelling algorithm and the intact 3D femur model were developed for this study and were validated independently before being applied to the final resurfacing model. The remodelling subroutine was tested using a simple loading case to test its performance during an Abaqus FE analysis involving the C3D10M elements, as used in the actual remodelling simulation. In addition, the FE femur model was calibrated and validated with respect to mechanical tests performed on a cadaveric specimen. On their own, each aspect of the analysis performed in a manner suggesting reliability and accuracy; however, the analysis involving the resurfaced femur includes complex geometry, contact interactions, varying material properties, and a non-uniform reference stimulus. The influence of these factors are discussed below. 


\subsection{1 - Mesh and Material Properties}

The finite element mesh used in the final model of this study was developed under a number of restrictions. The element selection was limited by geometric complexity and surface-to-surface contact modelling. As mentioned in Section 2.3.2.1, the selection of C3D10M elements was motivated at least in part by their common use in similar studies $[77-80]$. Throughout the development process, there was concern that the asymmetric nature of a tetrahedral mesh would introduce artifacts in the remodelling process. This was evident in early iterations of the cantilever beam model used to validate the subroutine. Larger tetrahedral elements cause noticeable asymmetries in the mesh. When adjacent elements such as these are allowed to remodel at different rates, a stress concentration develops at the interface. In results depicting the absolute change in density throughout the resurfaced model, such as Figure 3.5 or the results in Appendix G, there was a possible artifact evident in the lower cortex of the neck. The results indicate resorption of the cortex; however, there were small regions adjacent to the darker resorption regions that may suggest a local increase in density. These features were determined to be extrapolation artifacts created in the Abaqus field output. Querying individual integration point values or plotting the data as an element-averaged quilt (Figure H1, Appendix H), shows that no elements in the lower cortex (below region VI) showed local increases in bone. This was true for all cases analyzed. 
The overall element count was limited by computation time, which was made more apparent by the computational demands imposed by the subroutine. Conversely, a sufficiently refined mesh was required to capture the variation in bone density throughout the model and to accurately trace bone remodelling. Local mesh refinement was complicated by the influence that drastic variation in element size had on the reliability of the remodelling subroutine. Mesh convergence was also complicated by the subroutine. A mesh fine enough to yeild accurate stress and strain results may not be fine enough to completely capture the effects of bone adaptation. Consequently, each level of mesh refinement required a number of remodelling steps. The final mesh was refined to ensure adequate representation of the initial density distribution throughout the model, but allowed for practical analysis run-times. Further refinement did not present increased accuracy in the initial density field compared to the results seen in Figure 3.4.

\subsection{2 - Contact Properties}

Introducing contact into the remodelling routine added more limitations to the analysis. There was always a risk that elements in contact with the stiff implant model would undergo excessive deformation or that contact iterations failed to converge. The chances of this were increased when element density changed drastically in these regions. Elements from the femoral model adjacent to the implant were most likely to experience significant stress shielding. This 
was confirmed by the results summarized in Figure 3.2 and Appendix F where regions under the implant cap showed a significant decrease in stimulus levels. Analysis steps that were preceded by significant changes in element densities near the interaction surfaces always required several extra iterations for step convergence. Later steps converged quickly, once the remodelling stimulus had remained fairly constant.

\subsection{3 - Initial Reference Stimulus}

The baseline model plays a crucial role in the success of the overall simulation. It governs the reference case, or "goal" stimulus level, for the bone remodelling routine. Unlike in the validation model with the cantilevered beam, the initial stimulus distribution in the head of the femur was highly complex. It showed a trend reminiscent of the density distribution of the intact femoral head (contrast the baseline images in Figure 3.2 and Figure 3.4). This was unsurprising, given that the trabecular structure within the femoral head evolved in order to transfer the applied head-loads directly to the lower femoral cortex. It was therefore encouraging that the natural stimulus field presented in the baseline model resembles this structure.

\section{2 - Bone Remodelling After Uncemented Resurfacing}

The results for all the cases analyzed can be summarized simply as a loss of bone quality in regions under and adjacent to the resurfacing implant. Some 
regions, such as the upper aspect of the femoral head, the upper and lower cortex of the neck, and the regions between the implant stem and the femoral neck, were of particular interest. The local changes in bone quality gave an indication of overall implant performance and may provide insight into the most likely modes of failure. In addition, it was useful to compare the performance of implants with a fixed metaphyseal peg to that of an implant with an unfixed peg through changes in the surface interaction models. Including a cement layer in regions that consistently show large amounts of bone loss may be advisable, given that resorption of bone in such regions (ie: region I in Figure 3.5) appears inevitable. The analysis suggests that bone tissue in this region of the femoral head undergoes severe resorption regardless of the nature of fixation. The use of limited bone cement in this area may provide improved fixation minimize resorption of adjacent bone.

\subsection{1 - Resorption Under the Implant Cap}

The most apparent effect that the resurfacing implant had on the bone density of the femoral head was the drastic reduction in bone density that occurred in the trabecular bone within the femoral head. In all three contact cases, the bone above the implant peg adjacent to the inner surface of the cap was reduced to the minimum value allowable $(0.01 \mathrm{~g} / \mathrm{cc})$. The resorption of bone in this region was likely an unavoidable consequence of cementless resurfacing. Regardless of the nature of surface interaction, the upper surface of the implant 
cap will shield the bone tissue below. Load can be transferred instead through the stiffer implant to the upper rim and the area below the peg.

When the implant surface was tied to the bone material, the trabecular bone in the femoral head no longer experienced the same deformation. The implant effectively contains the head in a stiff spherical structure and prevented the natural deformation and stress distribution that would otherwise occur. The bone in the femoral head was significantly less dense than the cortical bone of the shaft, and normally experienced a great deal of compressive strain. The presence of the implant prevented deformation, lowered strain, and induced bone resorption. When the interface surface interaction was governed by the friction property, the resorption was less severe. Much smaller regions within the trabecular bone were driven down to $0.1 \mathrm{~g} / \mathrm{cc}$. The friction contact permitted small sliding displacements between the implant and the bone surface, thus allowing for some deformation of the bone material adjacent to the implant. As made apparent in the full friction case in Figure 3.3, this also allows the remodelling routine to return the model to a stimulus state similar to that of the intact femur. Because the tied interface in the other two cases caused complete resorption of bone in the proximal head (pushed to the lower limit of $0.01 \mathrm{~g} / \mathrm{cc}$ ), they could not return to anatomical loading even after remodelling. 


\subsection{2 - The Effect of the Metaphyseal Peg}

Fixing the implant peg had the effect of increasing load transfer between the peg and the lower cortex of the femoral neck. In some sense, this feature mimicked the lower half of the trabecular structure observed in the intact femur model. Although this could be seen as advantageous, it comes at the cost of decreased loading of the trabecular bone between the upper cortex of the neck and the implant. In the intact femoral head, there exists a minor trabecular structure that connects the upper cortex of the neck to the larger stalactic trabecular feature. The smaller feature was identified by a small region of higher density trabecular bone in Figure 3.4. In both cases where the peg was not tied to the surrounding bone, this feature remained at least partially intact. In the case of the tied implant peg, a large decrease in density between the peg and the upper cortex showed the feature to be entirely resorbed. Loss of bone mass in this region may indicate the onset of femoral neck narrowing.

The implant peg also caused bone hypertrophy in more distal regions of the head and neck. Bone tissue in this region of the femur was unaccustomed to carrying large loads such as those applied by the implant. Both of the frictionbased cases and the tied case demonstrated the local increases in bone density; however, the tied case showed the increase to be distributed over a much larger area. To avoid element collapse, or issues with contact convergence, the tip of the implant was not included in the surface pairs. This is equivalent to lengthwise 
over-drilling of the peg hole. Had interaction been allowed at this location on the implant, it is likely that the hypertrophy would have been more severe.

\subsection{3 - Implant Redesigning Based on Observations}

Of the three conditions tested, the purely friction-based model demonstrated lowest overall decrease in bone quality. Unfortunately, this model lacks practicality. It was developed using published friction data for press-fit cementless implants under primary stability. The actual interference parameter, the average degree of overlap between the implant and the bone tissue, was not available. The model was therefore constructed assuming initial contact across the entire surface, but with no built-in interference or surface pressure distribution. Furthermore, the study used for reference concerned only primary implant stability [85]. All current femoral resurfacing implants are either cemented or coated to promote bone ingrowth on at least some contact surfaces, most commonly the inner surface of the cap. Modelling a resurfacing implant without any fixed surfaces serves only to provide insight into the remodelling process when local bone tissue was slightly less constrained than in the tied cases. In practice, implants of this design would likely be unstable and vulnerable to aseptic loosening.

In light of the idea that increasing the tied (surface treated) area of an implant increases the stress shielding within the bone, a fourth test analysis was developed. In this case, the inner surfaces of the implant cap and peg were tied 
selectively to the bone tissue. Approximately one half the length of the implant peg, the proximal half, was modelled as a tied constraint. In addition, only a small area of the inner cap, directly adjacent to the peg, was tied (Figure 4.1). The remainder of the femoral cap was modelled with the pre-existing friction model and the distal aspect of the peg was ignored by removing any interaction model (friction or tied contact) it may have had with the femoral model. This is theoretically equivalent to shortening the implant peg without actually changing the model. Geometric changes to the implant would require a new mesh, new densities, and a new baseline model. By ignoring the lower region of the implant peg, it carried no load, and did not interact with the surrounding tissue. The femoral model was left with a pre-drilled peg hole that was almost twice as deep as required for the peg. Regardless, this model provided a promising compromise between the tied model and the friction model.
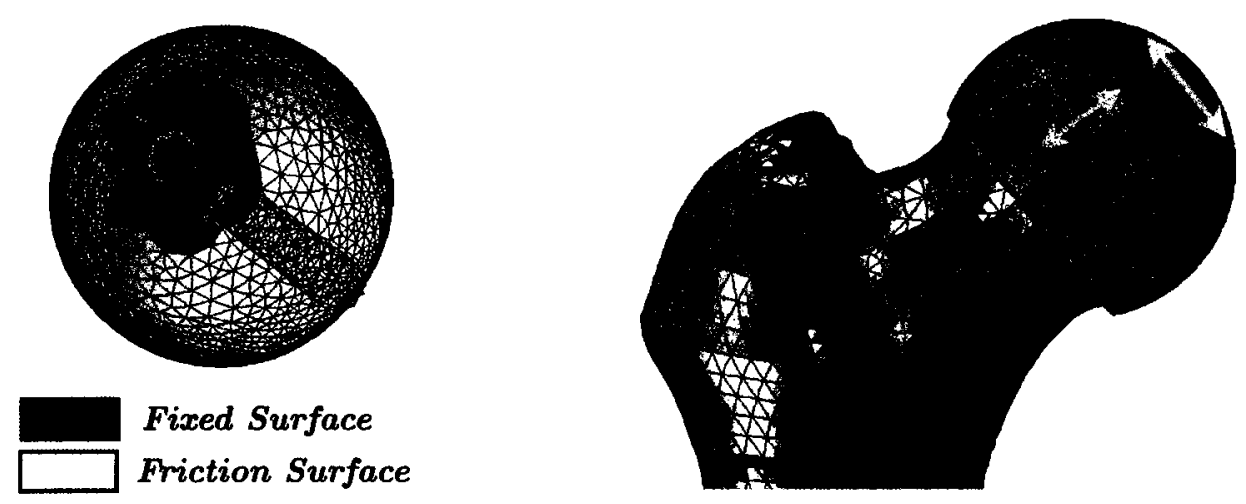

Figure 4.1: Surface contact area for selectively fixed model. Regions identified by the red shading (left) and the pink arrows (right) are fixed to the boney surfaces. The remainder of the inner surface of the femoral head was modelled using friction while the distal end of the peg remains entirely without surface restriction. 
The absolute change in density across the selectively fixed model suggested that by shortening the peg, and encouraging in-growth into the surrounding tissue, some of the negative effects observed in the other tied models can be avoided. Within the trabecular bone of the femoral head below the peg (region IV), there was an increase in local bone density (Figure 4.2). This mimicked the lower aspect of the trabecular structure present in the intact femur. The proximal region below the peg, region II, remains relatively unaffected, similar to the friction model. The model also exhibited less stress shielding in the bone tissue in region $\mathrm{V}$. The shortened peg did not transmit the load directly into the
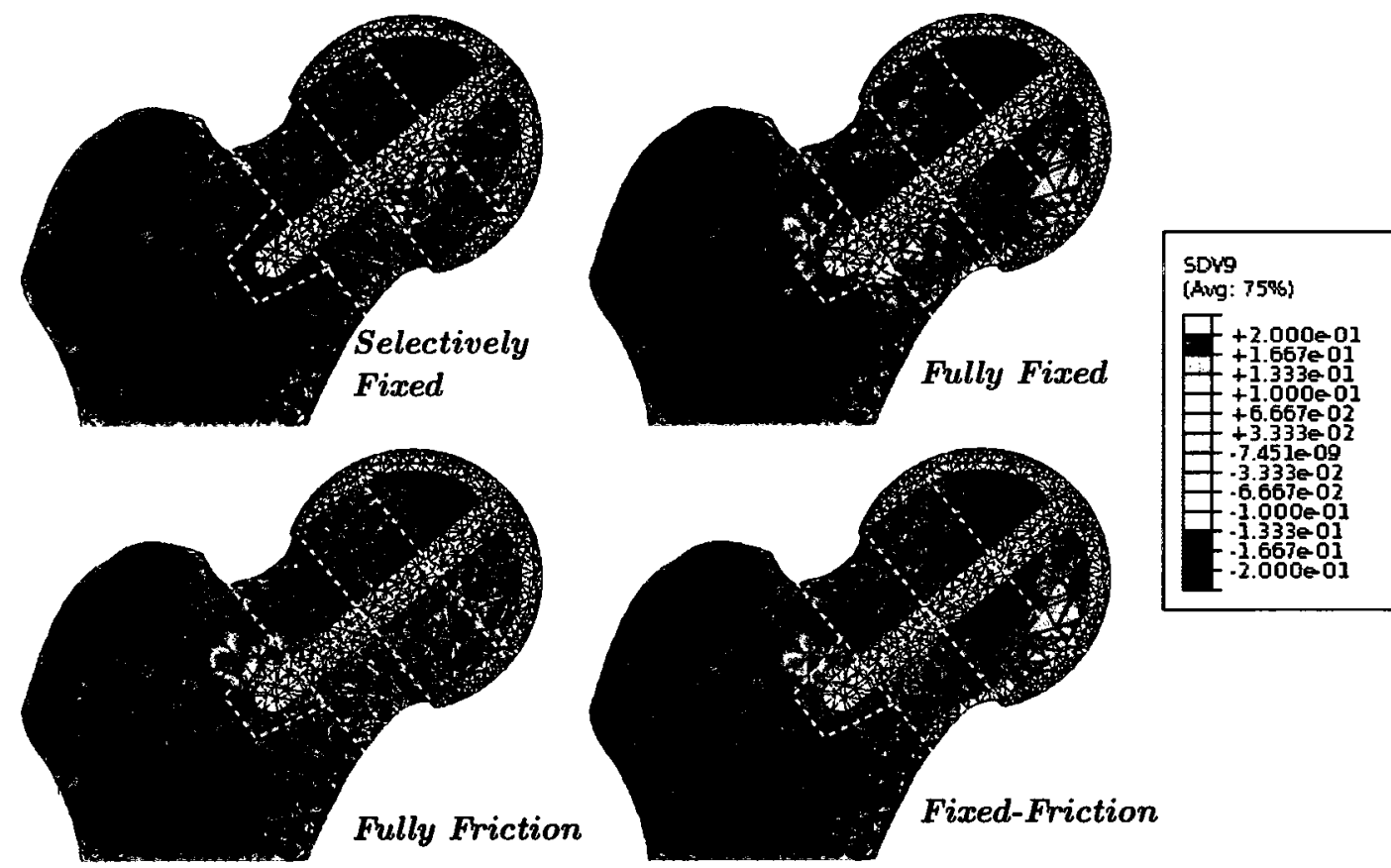

Figure 4.2: Final change in bone density under selectively fixed implant. Resorption of bone within the femoral head is minimized, and a trabecular structure develops between the implant peg and the lower cortex. Thinning of the cortical bone is still evident and significant resorption occurs in the upper regions of the femoral head. The original three contact cases are included for comparison. Values are in $\mathrm{g} / \mathrm{cc}$. 
neck, as in the original tied scenario. Unfortunately, region I of the femoral head still demonstrated significant reduction in local bone density, and the localized regions of bone density decrease along the cortex, thought to represent thinning of the cortical bone, were still present.

\section{3 - Comparison to Similar Studies}

Modern metal-on-metal hip resurfacing implants are in a relatively early phase of clinical trial and development. Only in the past 10 years has the use of these implants become more common. As such, most surgical studies outlining the successes and failures of these implant designs are only recently becoming available, and in many cases they present only short term results. Most studies involve exclusively cemented femoral resurfacing components; however, Springer et al. [86] have published a pool of data for cementless hip arthroplasty including clinical results for both THA and HRA. The results of this thesis can be qualitatively compared to short and longer term surgical outcomes of hip resurfacing arthroplasty from Springer et al. [86], and both qualitatively and quantitatively compared to analytical results presented by Gupta et al., Little et al., and Long et al. [2, 15, 61].

\subsection{1 - Recent Publications and Results}

In terms of surgical outcome, most failures of the femoral side component are associated with loosening of the component and femoral neck fracture $[1,3$, 
45]. Loosening of the implant was a consequence of poor primary stability often associated with improper application of cement or loss of bone quality inside the femoral head (avascular necrosis). Femoral neck fracture implies either increased strain or decreased bone quality in the neck of the post-operative resurfaced femur. It had also been suggested that mechanical factors that arise during surgery (such as neck notching, lengthening of the neck, and improper alignment) can cause neck fracture; however, the study performed by Steffen et al. [3] suggested neck fracture to be independent of these factors. Following this conclusion suggests that the neck fracture are likely due to changes in strain or changes in bone density.

Two separate finite element studies were performed by Little et al. [61] and Long et al. [2] in an effort to determine the changes in strain felt by bone tissue within the femoral neck. In 2007 , Little et al. found that there was very little change in the compressive and tensile strains in a cemented resurfaced femur relative to an intact model [61]. Contrary to the conclusions published by Little et al., in 2009 Long et al. published a study reporting that resurfacing may increase the compressive strain in the femoral neck up as much as $25 \%$ [2]. It was therefore suggested that this increase in strain led to eventual fracture failure of the femoral neck. Where both these studies concern the short-term changes induced by resurfacing, Gupta et al. [15] included bone remodelling into a cemented resurfacing model. The analysis showed up to $90 \%$ bone resorption under the implant and decreases in density ranging between $5-47 \%$ in the later 
aspect of the head. Gupta also observed an increase in compressive strain localized to the proximal region of the femoral neck. Bone density increased around the inferior edge of the implant, and little change was observed in the bone density of the distal neck or femoral diaphysis. The increase in strain near the implant edge was at least partially alleviated by the remodelling process.

\subsection{2 - Qualitative and Quantitative Contrast}

The degree of bone resorption observed in the analyses presented in this thesis are similar to the cemented implant results presented by Gupta et al. [15]. Decreases in bone density ranging from $40 \%$ to over $95 \%$ were observed in the proximal regions of the head, adjacent to the implant surface. In some cases, trabecular bone was reduced from a local density of $0.10-0.15 \mathrm{~g} / \mathrm{cc}$ to the minimum value of $0.01 \mathrm{~g} / \mathrm{cc}$. Had it be computationally allowable, it is likely that these regions would have seen a complete reduction of bone density. In cases where the implant stem was press fit, very little change in bone quality was observed within the femoral neck. This was in accordance with Gupta et al. [18]; however, some regions of the proximal cortex experienced reductions of $0.30 \mathrm{~g} / \mathrm{cc}$. Although this may only correspond to a reduction in density of $15-20 \%$, it was present in all the analysis cases tested and could be an indication of necking. The results for cementless resurfacing pooled by Springer et al. [86] showed that failure of the femoral component, through neck fracture or aseptic loosening, represents $70 \%$ of all resurfacing failures in after a 3.9 year followup. In addition, 
in a study of 610 resurfaced hips, femoral neck narrowing was observed in $30 \%$ of cases. Qualitative results present in this thesis certainly suggest that a decrease in bone density around the femoral neck is a likely concern.

Fixing the distal region of the implant stem caused severe reduction in bone quality in the upper aspect of the femoral neck. This effect was not observed by Gupta et al. [18], although it may be analogous to the findings by Little et al. regarding cemented pegs [61]. A cemented peg bears greater loads to the distal regions of stem, and therefore increases the potential for stress shielding. The analysis with a fixed stem shows this effect in the uncemented design.

A local strain concentration developed around the lower lip of the implant in both cases with the tied implant cap. In an identical manner to the findings of Gupta et al. [18], the strain in this area was alleviated through the remodelling process. This resulted in an increase in local bone density around the lower edge of the implant. 


\section{Chapter 5}

\section{Conclusions}

Four different simulations were performed using a 3D finite element model of a human femur to investigate the effects of bone remodelling inside a cementless femoral hip resurfacing implant. The principal model was developed from CT scans of a cadaveric femur specimen, and was ultimately calibrated against the cadaveric specimen itself. Implementing a theoretical bone remodelling algorithm permitted investigation into the effects of stress shielding.

Three initial implant interface conditions demonstrated drastic changes in bone density after several remodelling iterations. This adaptation was motivated by changes in the remodelling stimulus level, ultimately correlating to changes in strain energy density, between the intact femur model and the resurfaced models. Trabecular bone within the femoral head experienced a reduction in density between $40 \%$ and $95 \%$ of the initial values. Resorption was particularly apparent in regions above the peg, and in cases when the implant cap was tied to the adjacent bone.

Rigidly fixing the metaphyseal stem, which causes it to carry greater loads, had drastic effects on the surrounding bone. Bone between the distal stem and the lower cortex of the femoral neck saw a drastic increase in density. The density of bone between the peg and the upper neck of the femur decreased significantly. This may be considered an indication of femoral neck narrowing; however the drastic changes in density were limited to trabecular bone. In all 
analyses cases, the cortex of the neck showed decreases in density of up to $0.30 \mathrm{~g} /$ cc. This may also be an indication of neck narrowing.

The model without tied interfaces, involving only friction between the bone and the implant, presented the most favourable results. The tied interactions completely restrained local bone from undergoing normal deformation (strain) and this had a large influence on the remodelling. Although the friction model minimizes the effects of stress shielding, an implant of this design would be at a significant risk of aseptic loosening and instability. A fourth interaction analysis was therefore developed to reduce the stress shielding in the femoral head, while still selectively tying implant surfaces to provide stability.

It was determined that fixing only the proximal half of the implant stem and ignoring the distal end (effectively shortening the stem itself) reduced the area of the inner head that was affected by drastic bone resorption. In addition, only the most proximal regions of the cap surface, adjacent to the peg, were tied to the bone tissue. This leaves the outer rim of the implant to be in frictional contact with the bone, and alleviated the stress concentration that developed in this region of the other models. This case demonstrated reduction of the cortex of the femoral neck, and shielding in the proximal head, directly below the applied load.

Based on these findings, as well as by those presented by others in the literature, it would be beneficial to investigate the effect of shortening the stem of hip resurfacing implants - both cemented and uncemented. Allowing distal 
regions of the stem to carry load only increases stress shielding in the neck and head. Instead, fix the stem more proximal to the cap, near the center of rotation of the femoral head. Connecting the implant to the lower cortex of the neck and decreases stress shielding and stress concentration in the lower half of the femoral head. In addition, surfaces to be coated for bone in-growth should be selected carefully. Leaving regions near the rim of the implant cap unfixed was shown to also decrease strain concentration in the proximal femoral neck and aid in reducing stress shielding.

\section{1 - Contributions}

This thesis involved the construction of a 3D finite element model of a resurfaced proximal human femur from $\mathrm{qCT}$ scans. The intact femur model was assigned density based material properties and validated against a mechanical test of the cadaveric femur specimen. The results presented are novel in that they investigate the effects that cementless femoral resurfacing implants have on bone tissue around the implant while varying surface interaction characteristics. The model provided insight to the design of modern metal-on-metal resurfacing devices including the consequences of fixing the implant peg and of varying the fixed region within the implant cap. 


\section{References}

[1] P. R. Kim, P. E. Beaulé, G. Y. Laflamme, and M. Dunbar, "Causes of Early Failure in a Multicenter Clinical Trial of Hip Resurfacing," Jourmal of Arthroplasty, vol. 23, no. 6, suppl. 1, 2008.

[2] P. Long, T. J. Santner, and D. L. Bartel, "Hip Resurfacing Increases Bone Strains Associated with Short-Term Femoral Neck Fracture," Wiley InterScience, March 2009.

[3] R-T. Steffen et al., "Femoral Neck Fractures After Hip Resurfacing," Journal of Arthroplasty, vol. 24, no. 4, pp. 614-619, 2009.

[4] K. Bridwell, (2010). "Anatomical Planes of the Body" [WebPage]. Available: http://www.spineuniverse.com/anatomy/anatomical-planes-body

[5] M. Nordin and V. H. Frankel, Basic Biomechanics of the Musculoskeletal System, 3rd edition, Lippincott Williams and Wilkins, 2001.

[6] W. C. Whiting and R. F. Zernick, Biomechanics of Musculoskeletal Injury, 2nd Edition, Whiting and Zernick, 2008.

[7] C. Ethier and C. Simmons, Introductory Biomechanics: From Cells to Organisms, Cambridge, 2007.

[8] R. B. Martin, D. B. Burr, and N.A. Sharkey, Skeletal Tissue Mechanics, Springer, 1998. 
[9] D. W. Thompson, On Growth and Form: The Complete Revised Edition, Dover Publications, New York, 1992.

[10] D. R. Carter and W. C. Hayes, "The compressive behaviour of bone as a twophase porous structure," Journal of Bone and Joint Surgery Am., vol. 59, pp. 954-962, Oct 1977.

[11] L. Bassett and C. Andrew, "The Effect of Electrical Currents on Bone Structure," Proceedings of the Symposia Held at the Forty-third General Meeting of the International Association for Dental Research, Toronto Ontario, July 1965.

[12] V.H. Frankel and A.H. Burstein, Orthopaedic Biomechanics, Lea and Febiger, 1970

[13] J. H. Keyak, J.M Meagher, H.B. Skinner, and C.D. Mote, "Automated threedimensional finite element modelling of bone: a new method," Journal of Biomedical Engineering, vol. 12, pp. 389-397, 1990.

[14] J. H. Keyak, I.Y. Lee, and H.B. Skinner, "Correlation between orthogonal mechanical properties and density of trabecular bone," Journal of Biomedical Material Research, vol. 28, pp. 1329-1336, 1994.

[15] S. Gupta, S.M.R. New, and M. Taylor, "Bone remodelling inside a cemented resurfaced femoral head," Clinical Biomechanics, vol. 21, pp. 594-602, Jan 2006 . 
[16] H. Weinans, R. Huikes, and H.J. Grootenboer, "Effects of material properties of femoral hip components on bone remodeling", Journal of Orthopaedic Research, vol. 10, pp. 843-853, 1992.

[17] T. M. Keaveny and W.C. Hayes, "Mechanical properties of cortical and trabecular bone," Bone, vol. 7, pp. 185-344, 1993.

[18] Consensus Development Conference, "Prophylaxis and treatment of osteoporosis," Osteoporosis International, pp. 114-117, 1991.

[19] A. Moroni, A. Hoang-Kim, "Current augmentation fixation techniques for the osteoporotic patient," Scandinavian Journal of Surgery, vol. 94, pp. 103-109, 2005.

[20] J. Goldhahn, N. Suhm, S. Goldhahn, and B. Hanson, "Influence of osteoporosis on fracture fixation," Osteoporosis Intermational, vol. 19, pp. 761-772, 2008.

[21] J. Wolff, The Law of Bone Remodeling, Verlag von August Hirchwald, Berlin, 1892.

[22] R. J. Raggatt and N. C. Partridge, "Cellular and molecular mechanisms of bone remodelling", Journal of Biological Chemistry, vol. 285, no. 33, pp. 25103-25108, August 2010.

[23] K. Matsuo and N. Irie, "Osteoclast-osteoblast communication," Archives of Biochemistry and Biophysics, vol. 473, pp. 201-209, 2008. 
[24] A. G. Robling and C. H. Turner, "Mechanical signaling for bone modeling and remodeling," Critical Reviews in Eukaryotic Gene Expression, vol. 19, no. 1, pp. 319-338, 2009.

[25] P. Proff and P. Romer, "Molecular mechanism behind bone remodelling: a review," Clinical Oral Investigations, vol. 13, pp. 355-262, 2009.

[26] H.K. Uhthoff, P. Poitras and D.S. Backman, "Internal plate fixation for fractures: short history and recent developments," Journal of Orthopaedic Science, vol. 11, no. 2, pp. 118-126, 2006.

[27] M.T. Manley and K. Sutton, "Bearings of the future for total hip arthroplasty," Journal of Arthroplasty, vol. 23, pp. 45-50, 2008.

[28] M. Leonard, L. Mokotedi, U. Alao, A. Glynn, and M. Dolan, "The use locking plates in proximal humeral fractures," Journal of Shoulder Surgery, vol. 3, no. 4, pp. 85-89, 2009.

[29] B. Krolner and B. Toft, "Vertebral bone loss: an unheeded side effect of bedrest," Clinical Science, vol. 64, pp. 537-540, 1983.

[30] B. E. Nilsson and N. Westlin, "Bone density in athletes," Clinical Orthopaedics, vol. 77, pp. 179, 1971.

[31] Columbia University Medical Center, (2010). "Rehabilitation and Regenerative Medicine" [WebPage]. Available: http://www.cumc.columbia.edu/dept/rehab/ musculoskeletal_health/anatomy.html 
[32] R. Johnson and G. L Smidt, "Measurement of hip joint motion during walking," Journal of Bone and Joint Surgery, vol. 51, pp. 1083, 1969.

[33] R. Johnson and G. L Smidt, "Hip motion measurements for selected activities of daily living," Clinical Orthopaedics, vol. 72, pp. 205, 1970.

[34] L. Draganich, T. P. Andriacchi, A.M. Strongwater, and J.O. Galante, "Electronic measurement of instantaneous foot-floor contact patterns during gait", Journal of Biomechanics, vol. 13, pp. 875, 1980.

[35] T. P. Andriacchi, G. B. Andersson, R.W. Fermier, D. Stern, and J.O. Galante, "A study of lower-limb mechanics during stair-climbing," Journal of Bone and Joint Surgery, vol. 62a, pp. 749, 1980.

[36] G. Bergmann, G. Deuretzbacher, M. Heller, F. Graichen, A. Rohlmann, J. Strauss and G. N. Duda, "Hip contact forces and gait patterns from routine activities," Journal of Biomechanics, vol. 34, no. 7, pp. 859-871, 2001.

[37] P. Damm, and G. Bergmann, "Total hip joint prosthesis for in vivo measurement of forces and moments," Medical Engineering and Physics, vol. 32 , no. 1, pp. 95-100, 2001.

[38] R. D. Crowninshield, R. C. Johnson, and R. A. Brand, "The effects of walking velocity and age on hip during movement," Chir Organi Mov, vol. 132, pp. 140-144, 1978.

[39] H. Rohrle, R. Scholten, C. Sigolotto, W. Sollbach, and H. Kellner, "Joint forces in the human pelvis-leg skeleton during walking," Journal of Biomechanics, vol. 17 , pp. 409-424, 1984 . 
[40] A. J. van den Bogert, L. Read, and B.M Nigg, "Ana analysis of hip joint loading during walking, running, and skiing," Medicine and Science in Sports and Exercise, vol. 31, no. 1, pp. 131-142, 1999.

[41] Anne Benson-Gyles, (2007). "A letter to The Center for Devices and Radiological Health" [Email]. Made public by the FDA.

[42] D. R. Steinberg and M. E. Steinberg, "The early history of arthroplasty in the united states," Clin Orthop Relat Res, pp. 55-89, May 2000.

[43] H. C. Amstutz, P. E. Beaul, F. J. Dorey, M. J. L. Duff, P. A. Campbell, and T. A. Gruen, "Metal-on-metal hybrid surface arthroplasty: two to six-year follow-up study," J Bone Joint Surg Am, vol. 86A, pp. 28-39, Jan 2004.

[44] J. Daniel, P. B. Pynsent, and D. J. W. McMinn, "Metal-on-metal resurfacing of the hip in patients under the age of 55 years with osteoarthritis," $J$ Bone Joint Surg Br, vol. 86, pp. 177-184, Mar 2004.

[45] H. C. Amstutz and M. J. L. Duff, "Eleven years of experience with metal-onmetal hybrid hip resurfacing: a review of 1000 conserve plus," J Arthroplasty, vol. 23, pp. 36-43, Sep 2008.

[46] C. Heisel, J. Kleinhans, M. Menge, and J. Kretzer, "Ten different hip resurfacing systems: biomechanical analysis of design and material properties," Int Orthop, vol. 33, no. 4, pp. 939-943, Jul 2008.

[47] M. L. Buergi and W. L. Walter, "Hip resurfacing arthroplasty: the australian experience," J Arthroplasty, vol. 22, pp. 61-65, Oct 2007. 
[48] D. McMinn, R. Treacy, K. Lin, and P. Pynsent, "Metal on metal surface replacement of the hip: experience of the McMinn prothesis," Clin Orthop Relat Res, no. 329S, pp. S89-S98, Aug 1996.

[49] H. C. Amstutz, P. Grigoris, and F. J. Dorey, "Evolution and future of surface replacement of the hip," Journal of Orthopaedic Science, vol. 3, pp. 169-186, 1998.

[50] M. J. Quesada, D. R. Marker, and M. A. Mont, "Metal-on-metal hip resurfacing: Advantages and disadvantages," Jourmal of Arthroplasty, vol. 23, pp. 69-73, 2008.

[51] Zimmer Inc., (2010). "Durom Hip Resurfacing Product Information" [WebPage]. Available: http://www.zimmer.co.uk/z/ctl/op/ global/action/1/id/9226/template/MP

[52] A. Saha and S. Pal, "Mechanical properties of bone cement: a review," Journal of Biomedical Materials Research, vol. 18, pp. 435-462, 1984.

[53] L. C. Jones and D. S. Hungerford, "Cement disease", Clin Orthop Relat Res, vol. 225 pp. 192-206, Dec 1987.

[54] P. E. Beaulé, P. Campbell, and P. Shim, "Femoral head blood flow during hip resurfacing," Clin Orthop Relat Res, vol. 456, pp. 148-152, Mar 2007.

[55] T. P. Schmalzried, V. A. Fowble, K. J. Ure, and H. C. Amstutz, "Metal on metal surface replacement of the hip: technique, fixation, and early results", Clin Orthop Relat Res, no. 329S, pp. S106-S114, Aug 1996. 
[56] M. E. Berend, A. Smith, J. B. Meding, M. A. Ritter, T. Lynch, and K. Davis, "Long-term outcome and risk factors of proximal femoral fracture in uncemented and cemented total hip arthroplasty in 2551 hips," $J$ Arthroplasty, vol. 21, pp. 53-59, Sep 2006.

[57] J. S. M. de Aragn and K. S. Keisu, "21-year results of the uncemented fully textured lord hip prosthesis," Clin Orthop Relat Res, vol. 454, pp. 133-138, Jan 2007 .

[58] H. Amstutz and M. L. Duff, "Cementing the metaphyseal stem in metal-onmetal resurfacing: When and why," Clin Orthop Relat Res, vol. 467, no.1, pp. 79-83, Oct 2008.

[59] K. Brewster, (2007). "ActiveJoints.com, Hip Resurfacing Surgery" [WebPage]. Available: http://www.activejoints.com/resurfacing.html

[60] Smith \& Nephew, (2010). "Birmingham Hip, Technical Details" [WebPage]. Available: http://www.birminghamhipresurfacing.com/5

[61] P. Little, F. Taddei, M. Viceconti, D. W. Murray, and H. S. Gill, "Changes in femur stress after hip resurfacing arthroplasty: response to physiological loads," Clinical Biomechanics, vol. 22, pp. 440-448, May 2007.

[62] S. Spencer, R. Carter, H. Murray, and D. Meek, "Femoral Neck Narrowing after Metal-on-Metal Hip Resurfacing," Journal of Arthroplasty, vol. 23, no. 8, pp. 1105-1109, 2008. 
[63] P. Aspenberg, S. Goodman, S. Toksvig-Larsen, L.Ryd, and T. Albrektsson, "Intermittent micromotion inhibits bone ingrowth," Acta Orthopaedica, vol. 63, no.2, pp. 141-146, 1992.

[64] W. D. Bugbee and W. J. Culpepper, "Long term clinical consequences of stress-shielding after total hip arthroplasty without cement," Journal of Bone and Joint Surgery, vol. 79, pp. 1007-1012, 1997.

[65] K. Fischer, C. R. Jacobs, M. E. Levenston, and D. R. Carter, "Different loads can produce similar bone density distributions," Bone, vol. 19, pp. 127-135, 1996.

[66] R. A. Brand,C. M. Stanford, and C. C. Swan, "How do tissues respond and adapt to stresses around a prosthesis? A primer on finite element stress analysis for orthopaedic surgeons," Iowa Orthopaedic Journal, vol. 23, pp. 13-22, 2003.

[67] B. Behrens, I. Nolte, P. Wefstaedt, C. Stukenborg-Colsman and A. Bouguecha, "Numerical investigations on the strain-adaptive bone remodelling in the periprosthetic femur: Influence of the boundary conditions," Biomedical Engineering Online, vol. 8, pp. 7, 2009

[68] G. B. Sharma, R. E. Debski, P. J. McMahon, and D. D. Robertson, "Adaptive glenoid bone remodeling simulation," Journal of Biomechanics, vol. 42, pp. 1460-1468, 2009.

[69] D. Lin,Q. Li, W. Li, and M. Swain, "Dental implant induced bone remodeling and associated algorithms," Journal of the Mechanical Behaviour of Biomedical Materials, vol. 2, pp. 410-432, 2009. 
[70] P. K. Tomaszewski, N. Verdonschot, S.K. Bulstra, G.J. Verkerke, “A comparative finite-element analysis of bone failure and load transfer of osseointegrated prostheses fixations," Annals of Biomedical Engineering, vol. 38, no. 7, pp. 2418-2427, 2010.

[71] H. Weinans, R. Huiskes, B. van Rietbergen, D.R. Sumner, T.M. Turner, G.O. Galante, "Adaptive Bone Remodeling Around Bonded Noncemented Total Hip Arthroplasty: A Comparison Between Animal Experiments and Computer Simulation," Journal of Orthopaedic Research, vol. 11, pp. 500-513, 1993.

[72] D.R. Carter, "Mechanical loading histories and cortical bone remodelling," Calcified Tissue International, vol. 36, pp. S19-S24, 1984.

[73] OsiriX, open source development, (2010). "OsiriX Imaging Software" [WebPage]. Available: http://www.osirix-viewer.com,.

[74] Biomet Manufacturing Corp, (2010). "ReCap Femoral Resurfacing System" [WebPage]. Available: http://www.biomet.com/orthopedics/ productDetail.cfm?category $=1 \&$ product $=190$

[75] Biomet Orthopedics, Inc, (2010). "ReCap Femoral Resurfacing System: Surgical Techniques" [Demonstration manual]. Available: http:// www.biomet.com/orthopedics/getFile.cfm?id=1761\&rt=inline

[76] D.S. Simulia Corp., (2010). Abaqus 6.8 Theory Manual [Online documentation]. Available: http://abaqus.ethz.ch:2080/v6.8/books/stm/ default.htm 
[77] X. You, T. Connolley, P.E. McHugh, H. Cuddy, C. Motz, "A combined experimental and computational study of deformation in grains of biomedical grade 316LVM stainless steel," Acta Materialia, vol. 58, pp. 4825-4850, 2006.

[78] H. Fouad, "Effects of the bone-plate material and the presence of a gap between the fractured bone and plate on the predicted stresses at the fractured bone," Medical Engineering and Physics, vol. 23, no. 7, pp. 783-789, 2010.

[79] Q. Li, L. Zheng, Z Zhang, and Z Cai, "Biomechanical evaluation of lumbosacral reconstruction after subtotal sacrectomy: A three-dimensional finite element analysis," Chinese-German Journal of Clinical Oncology, vol. 8, no. 11, pp. 638-641, 2009.

[80] S. Portnoy, Z. Yizhar, N. Shabshin, Y. Itzchak, A. Kristal, Y. Dotan-Marom, I. Siev-Ner, and A. Gefen, "Internal mechanical conditions in the soft tissues of a residual limb of a trans-tibial amputee," Journal of Biomechanics, vol. 41, pp. $1897-1909,2008$.

[81] Mindway's Software, Inc., "CT Calibration Phantom: User's Guide", San Francisco, 2002.

[82] S. Gupta, F. C. van der Helm, and F. van Keulen, "The possibilities of uncemented glenoid component - a finite element study," Clinical Biomechanics, vol. 19, no. 3, pp. 292-302, 2004.

[83] P.P. Benham, R.J. Crawford, and C.G. Armstrong, Mechanics of Engineering Materials, 2nd edition, pp. 310-312, Longman Group Limited, 1996. 
[84] M. E. Taylor, K. E. Tanner, M. A. Freeman, and A.L. Yettram, "Stress and Strain Distribution Within the Intact Femur: Compression or Bending?" Medical Engineering and Physics, vol. 19, no. 1, pp. 97, 99-100, 1996.

[85] A. Gebert, J. Peters, N.E. Bishop, F. Westphal, and M.M. Morlock, "Influence of press-fit parameters on the primary stability of uncemented femoral resurfacing implants," Medical Engineering and Physics, vol. 31, pp. 160-164, 2009.

[86] B. D. Springer, S.E Connolley, S.M. Odum, T.K. Fehring, W.L. Griffin, J.B. Mason, J.L. Masonis, "Cementless femoral components in young patients: review and meta-analysis of total hip arthroplasty and hip resurfacing," Journal of Arthroplasty, vol. 24, no. 6, suppl. 1, 2006. 


\section{Appendix A: Matlab Routine}

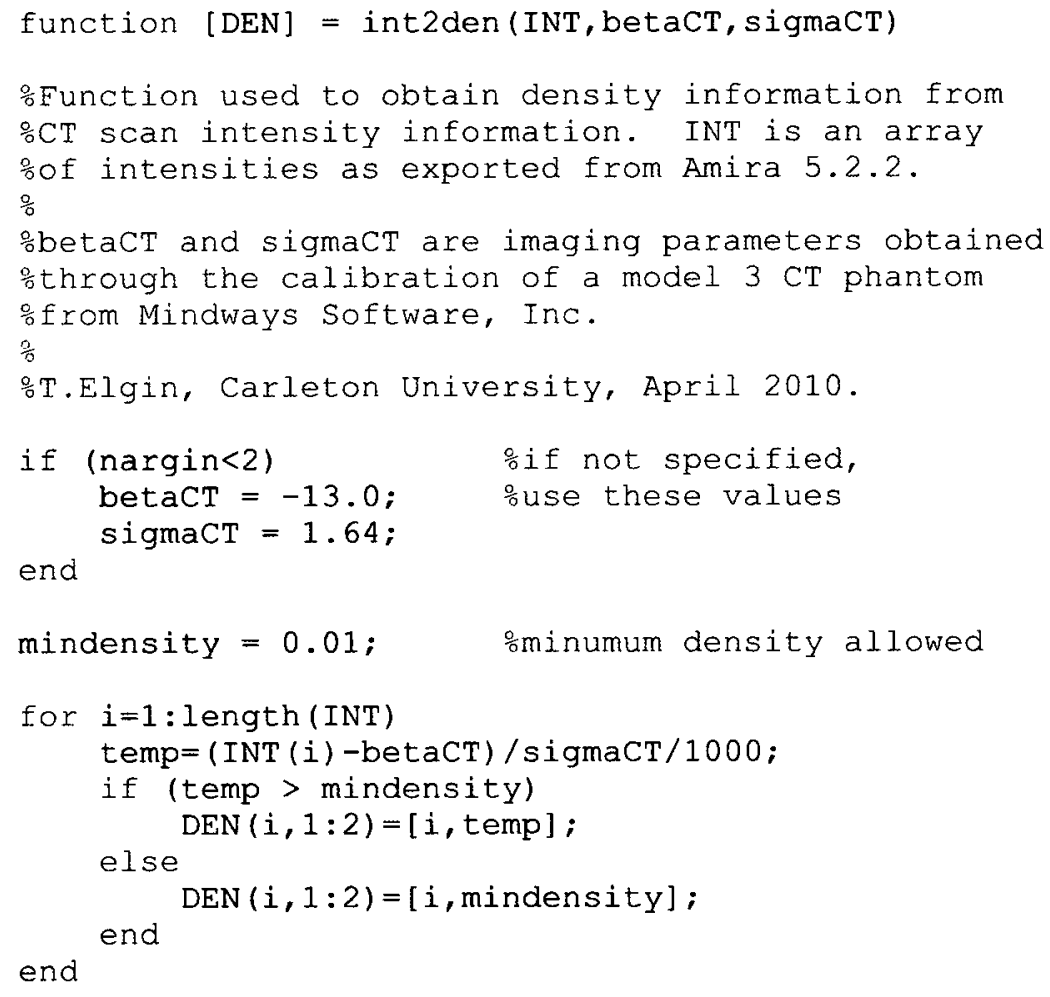




\section{Appendix B: Fortran Subroutines}

\section{B1: Baseline Routine}

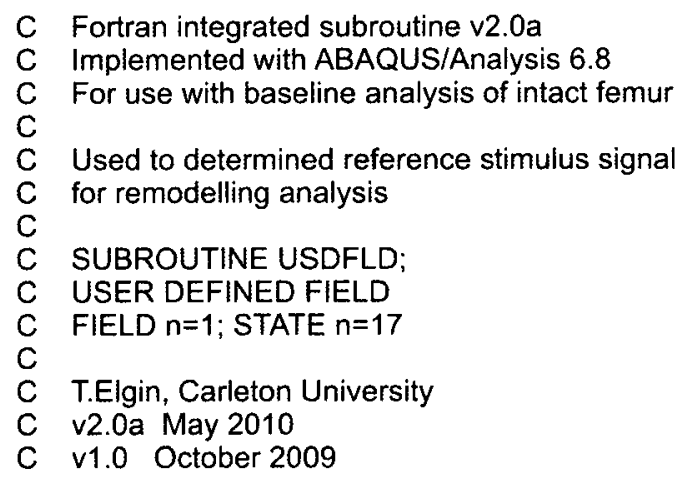

SUBROUTINE USDFLD(FIELD,STATEV,PNEWDT,DIRECT,T,CELENT, 1 TIME,DTIME,CMNAME,ORNAME,NFIELD,NSTATV,NOEL,NPT,LAYER, 2 KSPT,KSTEP,KINC,NDI,NSHR,COORD,JMAC,JMATYP,MATLAYO,

IStore stiffness in state var !initialization boolean is set to YES 


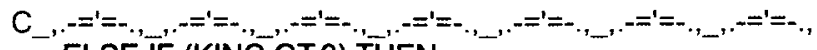

ELSE IF (KINC.GT.0) THEN

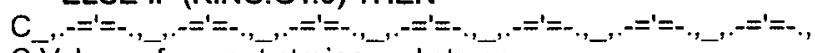

$C$ Values of current strains and stress:

CALL GETVRM('ENER',ARRAY,JARRAY,FLGRAY,JRCD,JMAC,JMATYP,MATLAYO,LACCFLA) $\mathrm{U}=\operatorname{ARRAY}(1)$

C Access past states

CALL GETVRM('SDV',ARRAY,JARRAY,FLGRAY,JRCD,JMAC,JMATYP,MATLAYO,LACCFLA)

STATEV $(1)=$ ARRAY (1) !stiffness

STATEV $(2)=$ ARRAY $(2) \quad$ !density

C Redefine RHO

RHO = STATEV $(2)$

$C$ Calculate strain energy density and stiffness for current element

$\mathrm{Sn}=\mathrm{U} / \mathrm{RHO}$

$\operatorname{STATEV}(3)=\mathrm{Sn}$

FIELD(1) $=7218^{*}\left(\mathrm{RHO}^{* *} 1.52\right)^{*} 1000000$

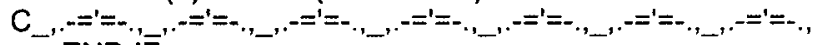

END IF

$\operatorname{STATEV}(4)=0$

STATEV $(5)=0$

STATEV $(6)=0$

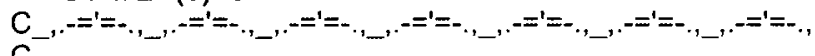

C Output initial condition file for remodelling analysis

C Includes: Element \#. Integration pnt. \#, Density, Reference stimulus

C

IF ((KSTEP $==2)$.AND. $($ KINC.EQ.1)) THEN

OPEN (12, FILE = "/home/telgin/ABAQUS/zFemur/CAL.txt", ACCESS = "APPEND")

WRITE(12,*) NOEL,NPT,RHO,Sn

CLOSE(12)

C

END IF

RETURN

$\mathrm{C}$

END 


\section{B2: Remodelling Routine}

C Fortran integrated subroutine $v 3.2 z$

C Implemented with ABAQUS/Analysis 6.8

C For use with remodelling analysis of resurfaced femur

C

C SUBROUTINE USDFLD;

C USER DEFINED FIELD

C FIELD $n=1 ;$ STATE $n=12$

C

C T.Elgin, Carleton University

C $\quad$ 3.2z May 2010

C $\quad$ 1.0 October 2009

SUBROUTINE USDFLD(FIELD,STATEV,PNEWDT,DIRECT,T,CELENT, 1 TIME,DTIME,CMNAME,ORNAME,NFIELD,NSTATV,NOEL,NPT,LAYER, 2 KSPT,KSTEP,KINC,NDI,NSHR,COORD,JMAC,JMATYP,MATLAYO,

C 3 LACCFLA)

C

INCLUDE 'ABA_PARAM.INC'

$\mathrm{C}$

CHARACTER ${ }^{*} 80$ CMNAME,ORNAME

CHARACTER ${ }^{*}$ FLGRAY(15)

DIMENSION FIELD(NFIELD),STATEV(NSTATV), DIRECT(3,3),

$1 \mathrm{~T}(3,3)$, TIME(2)

DIMENSION ARRAY(15),JARRAY(15),JMAC(*),JMATYP(*),

1 COORD(*)

C

DIMENSION Snn(24049)

C STATE IDENTITIES:

C 1 - STIFFNESS (E)

C 2 - CURRENT DENSITY (RHO)

C 3 - BASELINE STIMULUS (Sn)

C 4 - CURRENT STIMULUS (S)

C 5 - FEEDBACK RATIO $\left(S-(1-1+x)^{*} \mathrm{~S} n\right)$

C 6 -STEP CHANGE IN DENSITY (dRHO)

C 7 - INITIALIZATION BOOLEAN $(0=$ not initialized $)$

C 8 - BASELINE DENSITY (RHON)

C 9 - ABS CHANGE IN DENSITY (RELATIVE TO BASELINE)

C 10 - ABS CHANGE IN STIMULUS (RELATIVE TO BASELINE)

C 11 - PERCENT CHANGE IN DENSITY (RELATIVE TO BASELINE)

C 12 - PERCENT CHANGE IN STIMULUS (RELATIVE TO BASELINE)

C

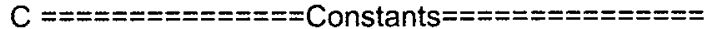

$E P S=1.0 \mathrm{E}-05$

C Min/Max dRHO values

RHOMAX $=2.5000$ !Max allowable density

RHOMIN $=0.0100 \quad$ ! Min allowable density

dRHOMAX $=0.0500$ !Max change in density dRHOMIN $=-0.0500$ !Min change in density

C Normal tolerance (absolute value) $x=0.1000 \quad$ IRatio $(+/-10 \%)$ for "lazy zone" $\mathrm{C}=0.000004$ !Remodelling rate constants

$\mathrm{C}============$ Initial States $=============$

CALL GETVRM('SDV',ARRAY,JARRAY,FLGRAY,JRCD,JMAC,JMATYP,MATLAYO,LACCFLA)

C This CALL returns all state variables into the ARRAY variable

C The states are then re-stored within the routine as STATEV $(n)$

$\operatorname{STATEV}(1)=$ ARRAY $(1) \quad$ !stiffness

STATEV (2) = ARRAY (2) !density

STATEV (3) $=$ ARRAY $(3) \quad$ !Sn

STATEV $(7)=\operatorname{ARRAY}(7) \quad$ !Initialized? (boolean) 


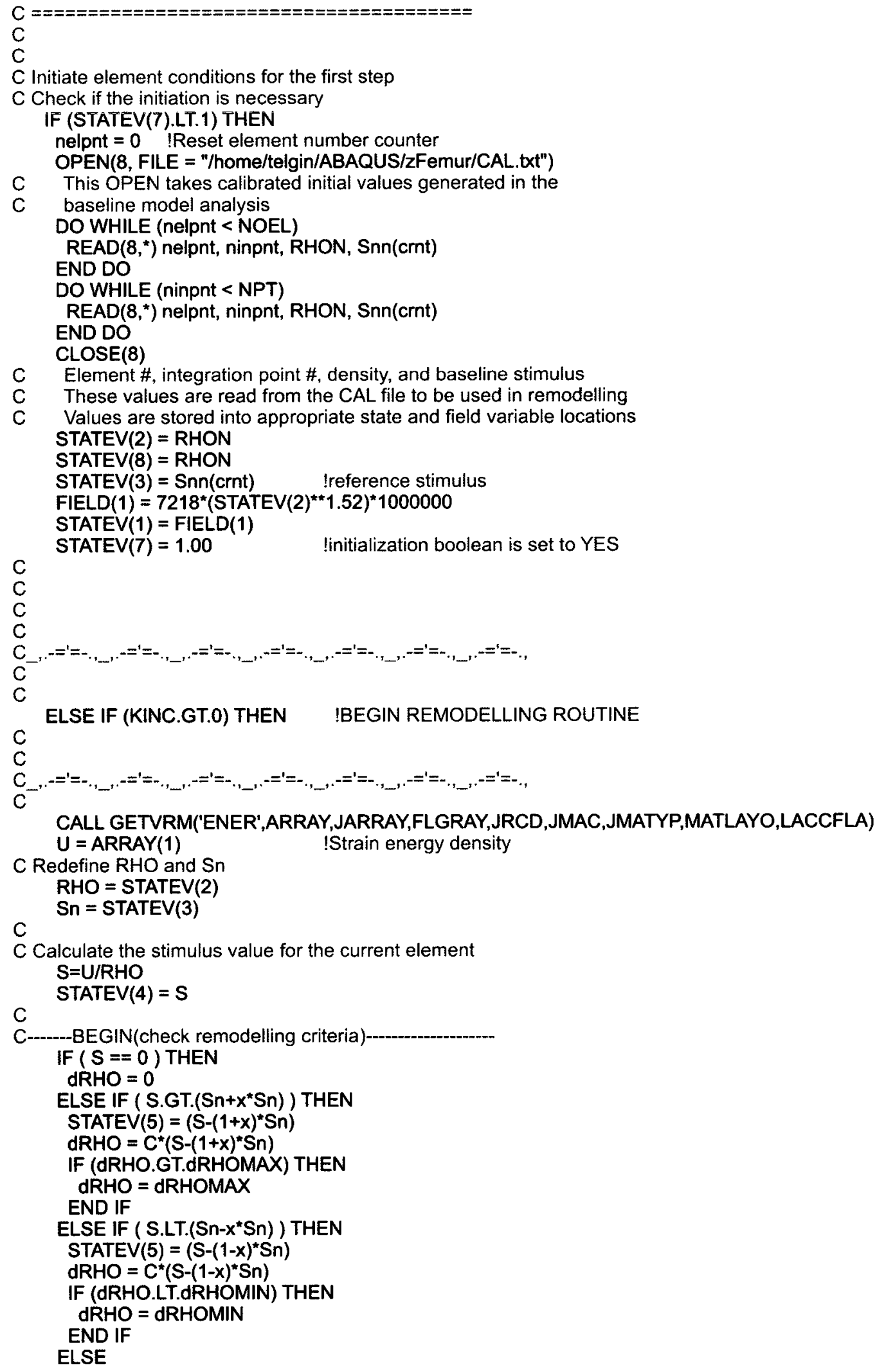




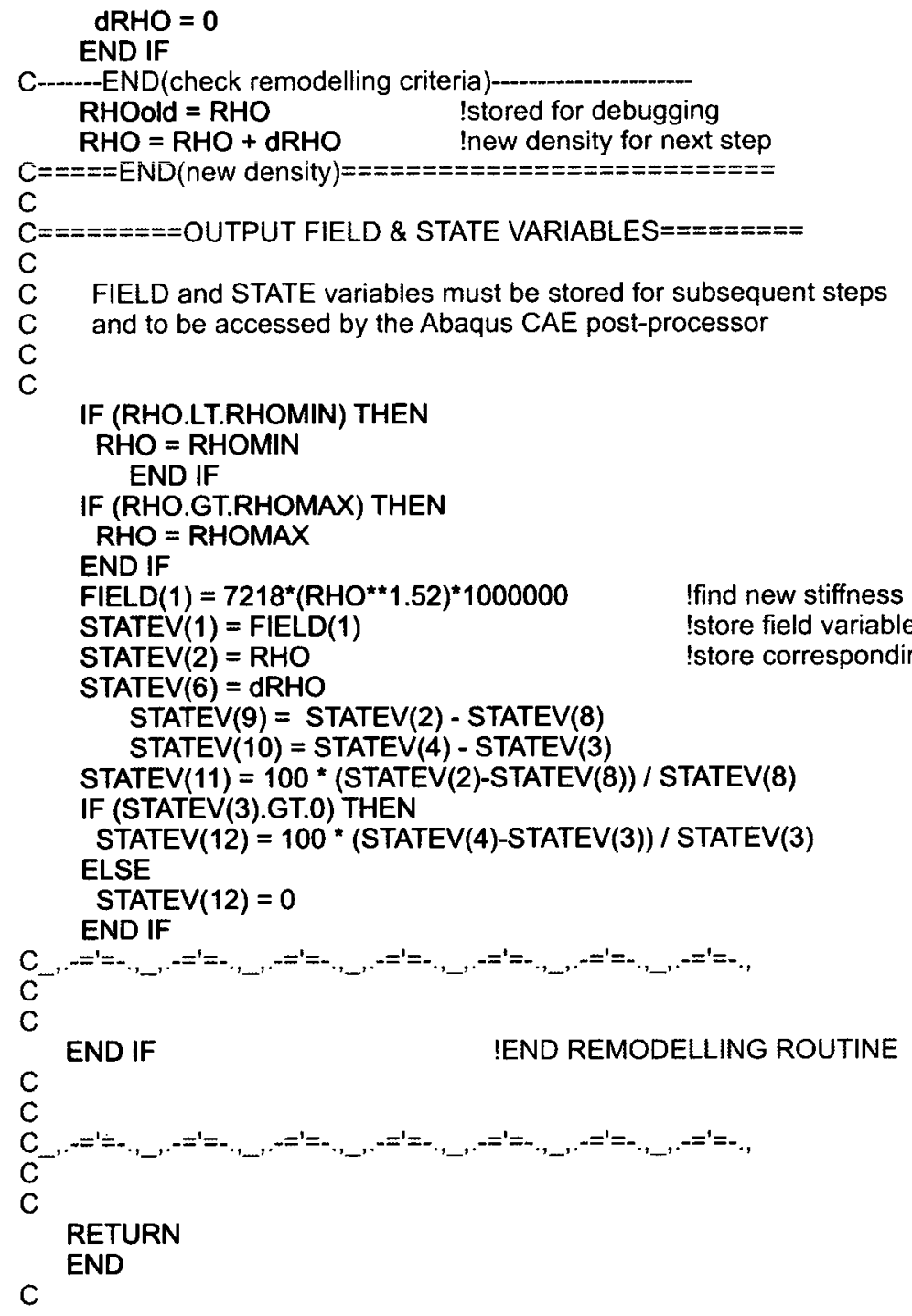




\section{Appendix C: Remodelling Validation Results}

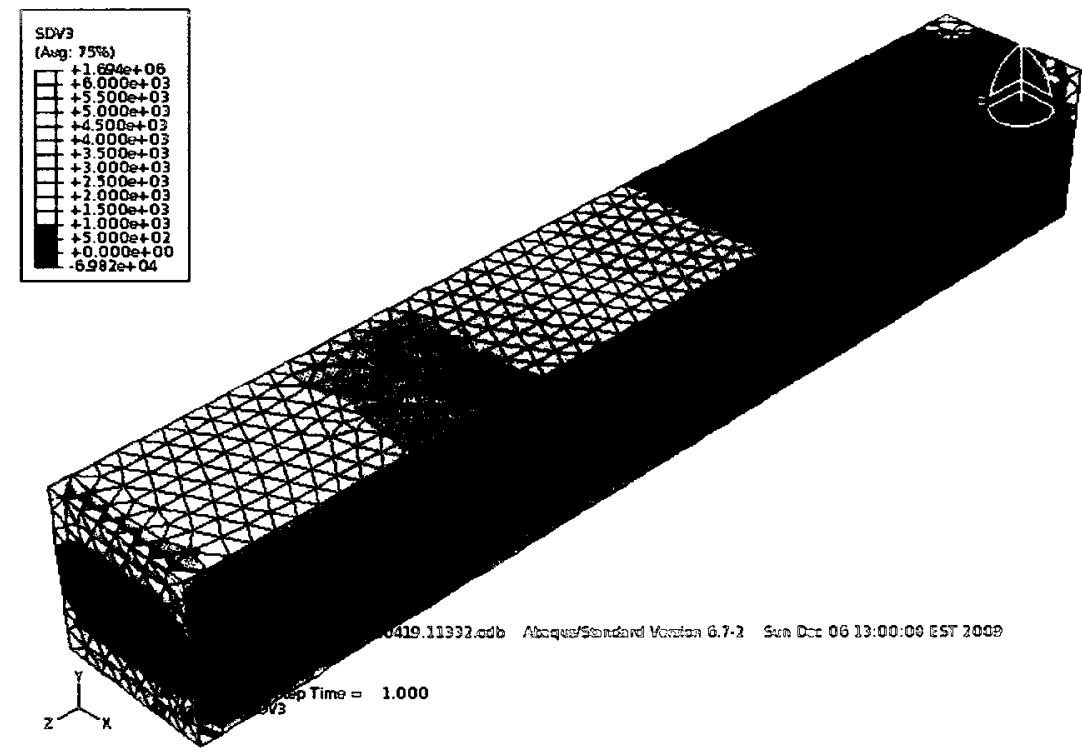

Figure C1: Baseline stimulus distribution in cantilever beam $(J / g)$.

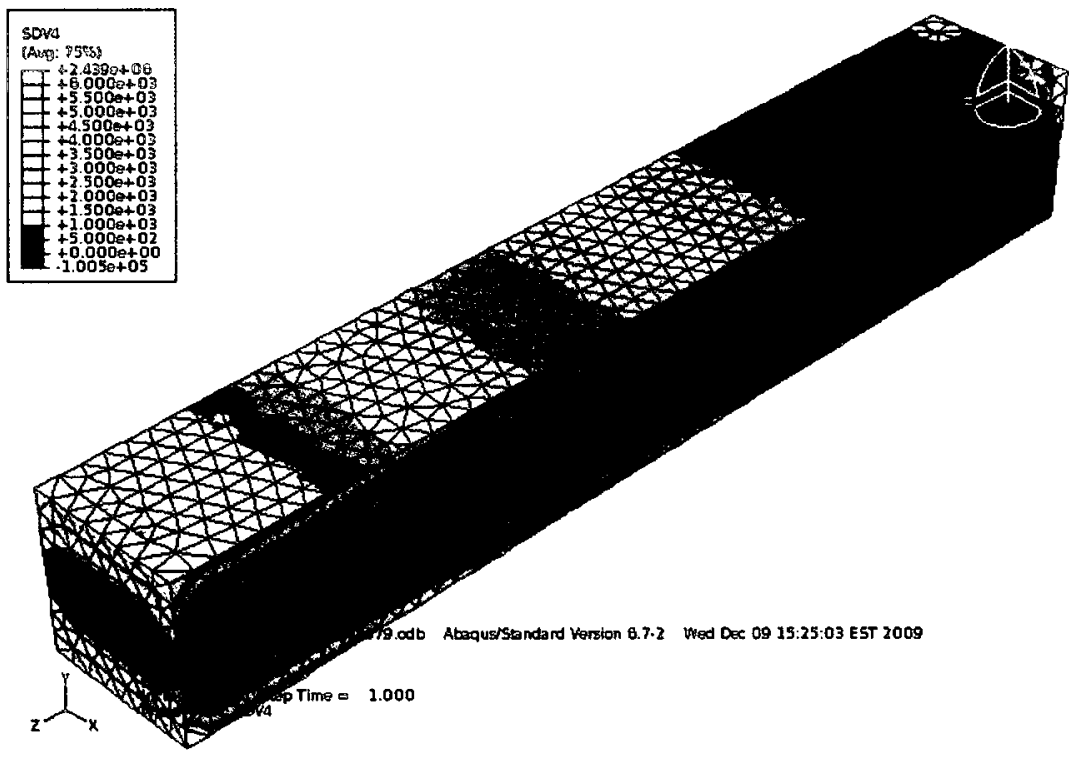

Figure C2: Stimulus distribution on step 2 of remodelling: 20\% increase in load $(J / g)$. 


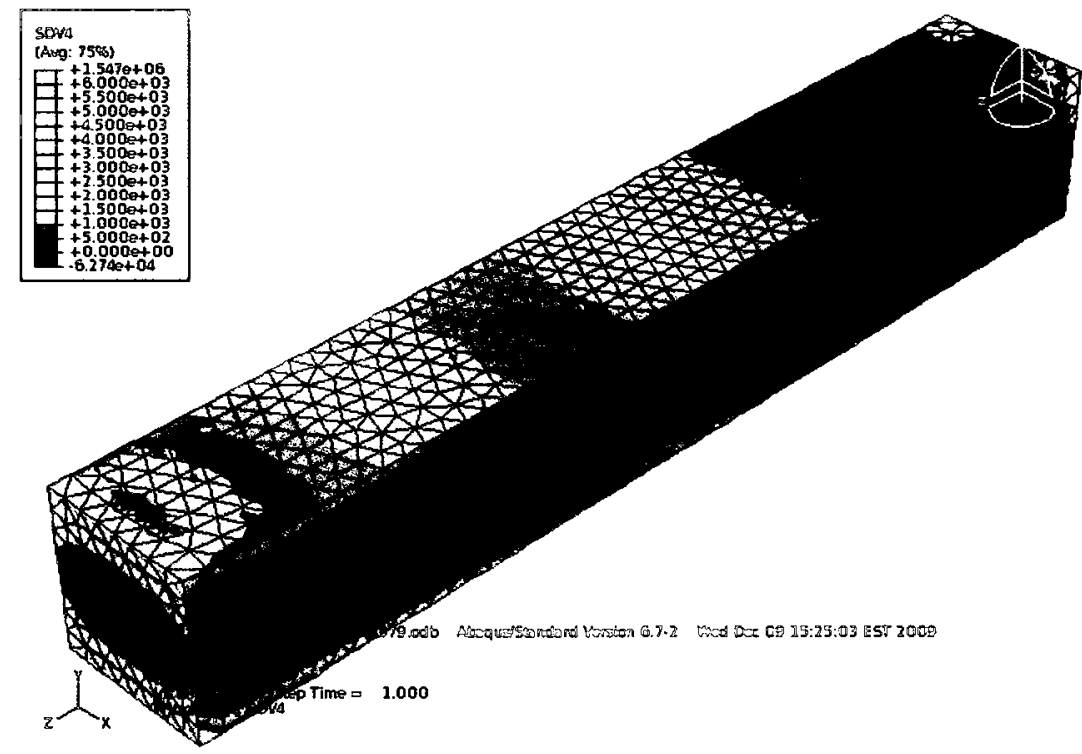

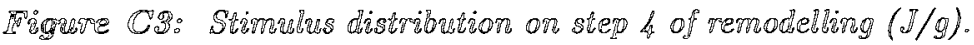

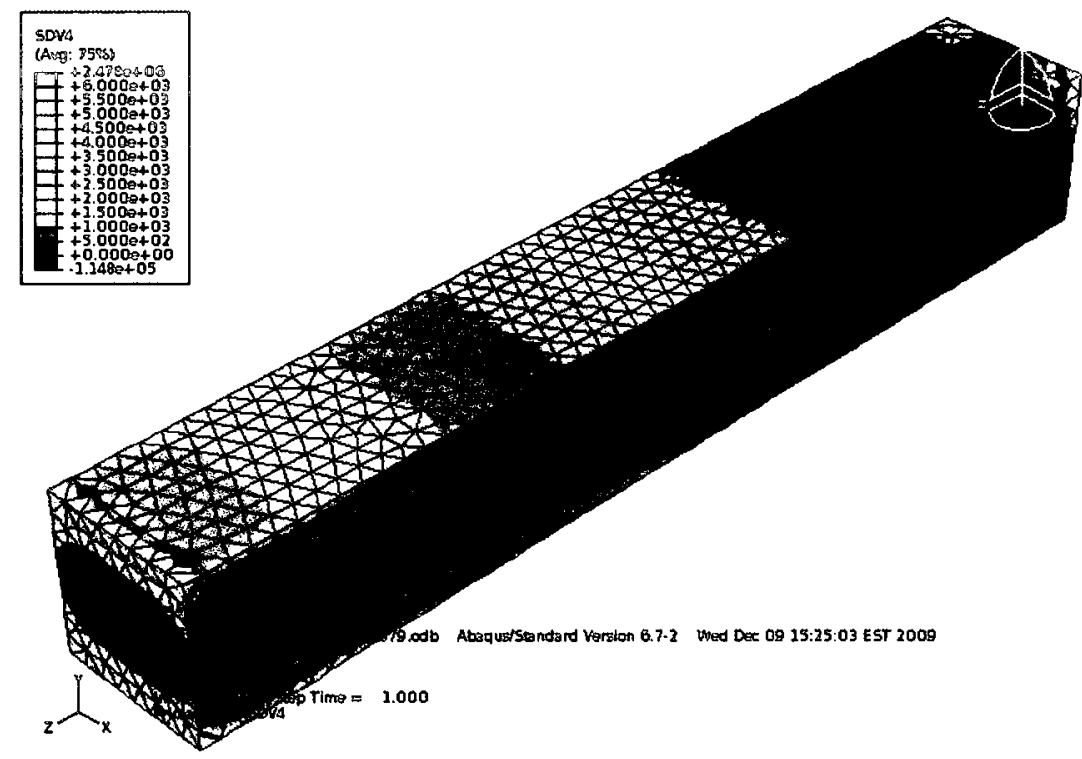

Figure C4: Stimulus distribution on step 10 of remodelling $(\mathrm{J} / \mathrm{g})$. 


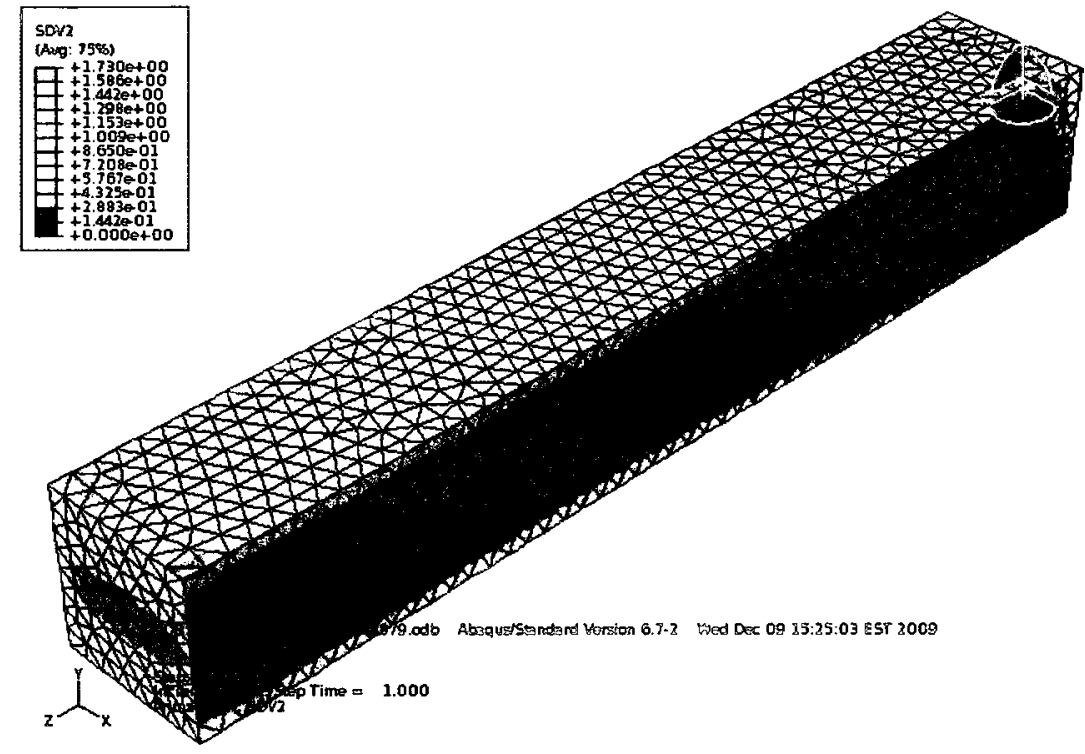

Figure Cy: Final density distribution after 10 th step of the remodellizg validation analysis. Greater density changes were observed in regions away from the neutral axis and towards the fixed end of the cantilevered beam ( $g$ / $c c)$. 


\section{Appendix D: Equipment and Apparatus}

\section{MTS Actuated Load Frame}

858 Mini Bionix II

Model: $359 \mathrm{~A} / \mathrm{T}$

SN: 10210302

Assembly No.: 56278102 Rev A

Vishay Instruments Division

P-3500 Strain Indicators ( $\mathrm{x} 2$ )

SN: 0140520 (Carleton U. ID: 505269)

SN: 98488 (Carleton U. ID: 041277)

SB-10 Switch and Balance Unit ( $x 2$ )

SN: 105099

SN: 0120086

TML Strain Gauges (Tokyo Sokki Kenkyujo Co., Ltd.)

Type FRA-1-11 general purpose rosettes

Arrangement: Three gauge, stacked, $45^{\circ} / 45^{\circ}$

Gauge length: $1 \mathrm{~mm}$

Gauge factors: $2.07 / 2.07 / 2.07 \pm 1 \%$

Resistance: $120 \pm 0.5 \mathrm{ohms}$

Lot No. A401231

\section{Polaris Stereo Camera System}

Polaris Hybrid Position Sensor

SN: P4-06094

with NDI Tool Tracker software 


\section{Appendix E: Data from Mechanical Testing}

\begin{tabular}{|c|c|c|c|c|c|c|}
\hline \multirow[b]{2}{*}{ Force (N) } & \multicolumn{3}{|c|}{ Set One } & \multicolumn{3}{|c|}{ Set Two } \\
\hline & 1500 & 1750 & 2000 & 1500 & 1750 & 2000 \\
\hline & \multicolumn{6}{|c|}{ Strain Gauge Readings (microstrain) } \\
\hline A1 & 440 & 554 & 302 & & & \\
\hline B1 & -50 & -44 & -4 & & & \\
\hline $\mathbf{C 1}$ & -450 & -526 & 33 & & & \\
\hline A2 & -649 & -784 & -945 & -742 & -791 & -902 \\
\hline B2 & -1968 & -2345 & -2780 & -2019 & -2209 & -2525 \\
\hline $\mathbf{C 2}$ & -409 & -514 & -618 & -615 & -610 & -684 \\
\hline \multicolumn{7}{|l|}{ Gauges } \\
\hline A3 & -2634 & -3123 & -3642 & -2610 & -3097 & -3596 \\
\hline B3 & -1385 & -1652 & -1932 & -1387 & -1656 & -1925 \\
\hline C3 & 224 & 273 & 314 & 252 & 273 & 319 \\
\hline A4 & -483 & -557 & -658 & -473 & -568 & -664 \\
\hline B4 & -1751 & -2056 & -2391 & -1699 & -2027 & -2369 \\
\hline C4 & -894 & -1040 & -1202 & -854 & -1019 & -1188 \\
\hline
\end{tabular}

Table D1: Strain data gathered from the cadaveric femur. All values are in microstrain. Gauge one became erroneous at the end of the first load set. It was ignored for the subsequent set. Figure 2.13 shows the locations and approximate orientations of each rosette gauge.

\begin{tabular}{|c|c|c|c|c|c|c|c|}
\hline \multirow{2}{*}{\multicolumn{2}{|c|}{ Force (N) }} & \multicolumn{3}{|c|}{ Set One } & \multicolumn{3}{|c|}{ Set Two } \\
\hline & & 1500 & 1750 & 2000 & 1500 & 1750 & 2000 \\
\hline \multirow{2}{*}{$\begin{array}{c}\text { Actuator Position } \\
\text { (mm) }\end{array}$} & At contact & 21.7 & 21.7 & 21.7 & 19.97 & 19.97 & 19.97 \\
\hline & Loaded & $n / a$ & 17.3 & 16.33 & 17.1 & 16.63 & 16.09 \\
\hline \multicolumn{2}{|c|}{ Net displacement at head (mm) } & $n / a$ & 4.4 & 3.64 & 3.34 & 3.34 & 3.88 \\
\hline
\end{tabular}

Table D2: Displacement data gathered from the cadaveric femur. The displacement of the femoral head when loaded was measured as the difference between the actuator position at initial contact with the femur, and the position at a given load. No value was taken at $1500 \mathrm{~N}$ in set one. 


\section{Appendix F: Density Variation During Analyses}
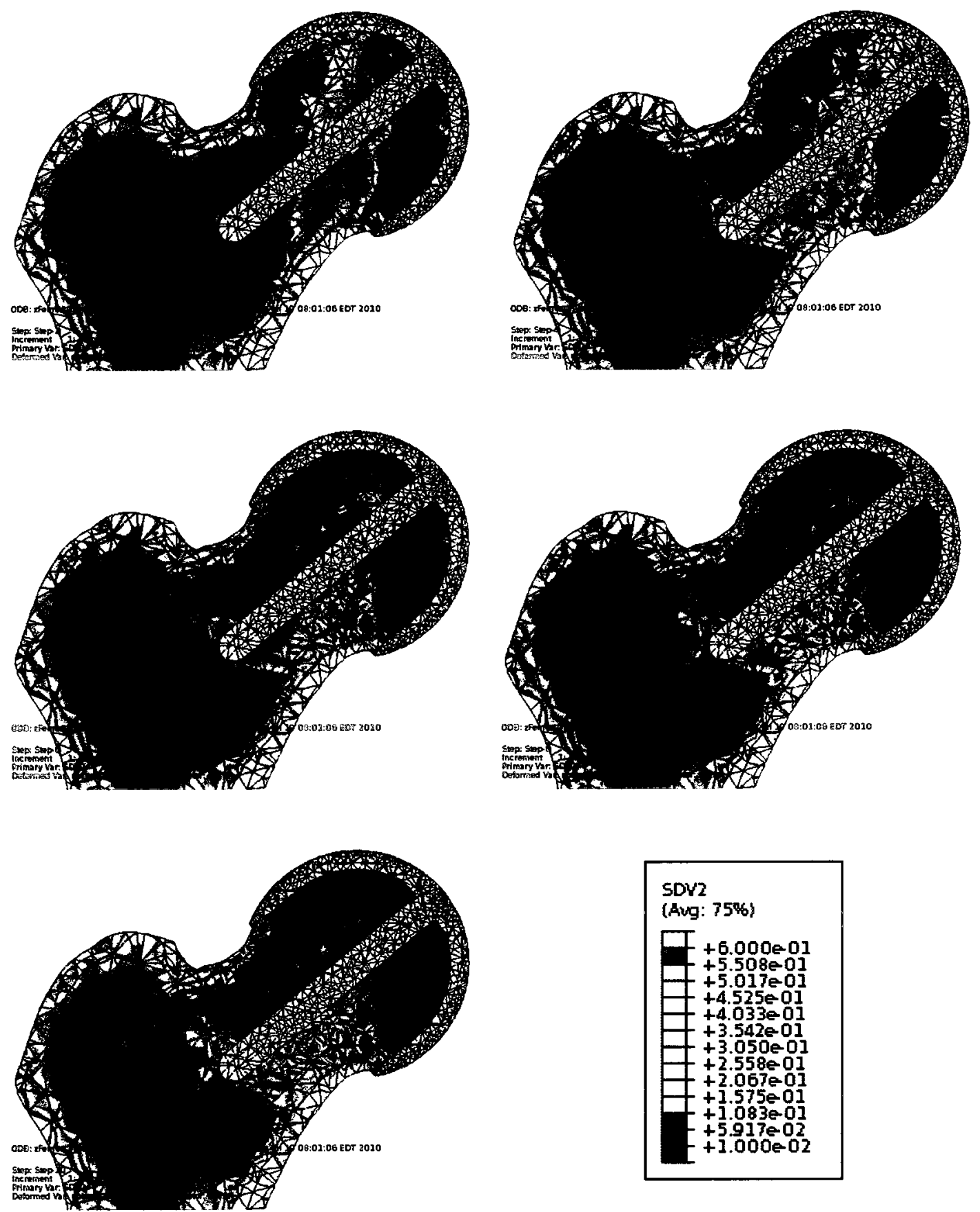

Figure F1: Bone density during analysis of the fully fixed model. From left to right, top to bottom: step 2, 4, 6, 8, 10. Density values are all in $\mathrm{g} / \mathrm{cc}$. 

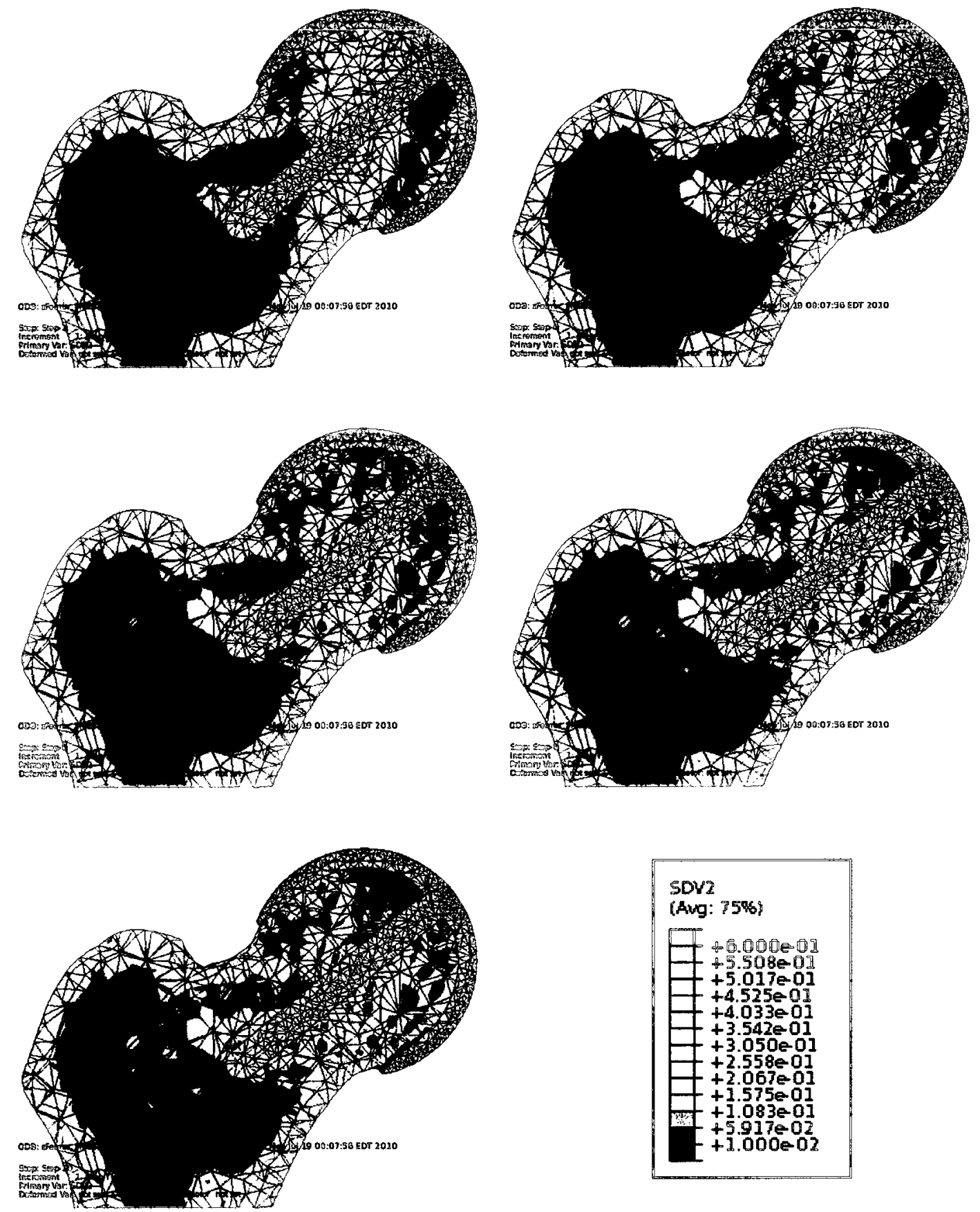

Figure F2: Bone density during analysis of the fully friction model. From left to right, top to bottom: step $\mathscr{2}, 4,6,8,10$. Density values are all in $\mathrm{g} / \mathrm{cc}$. 

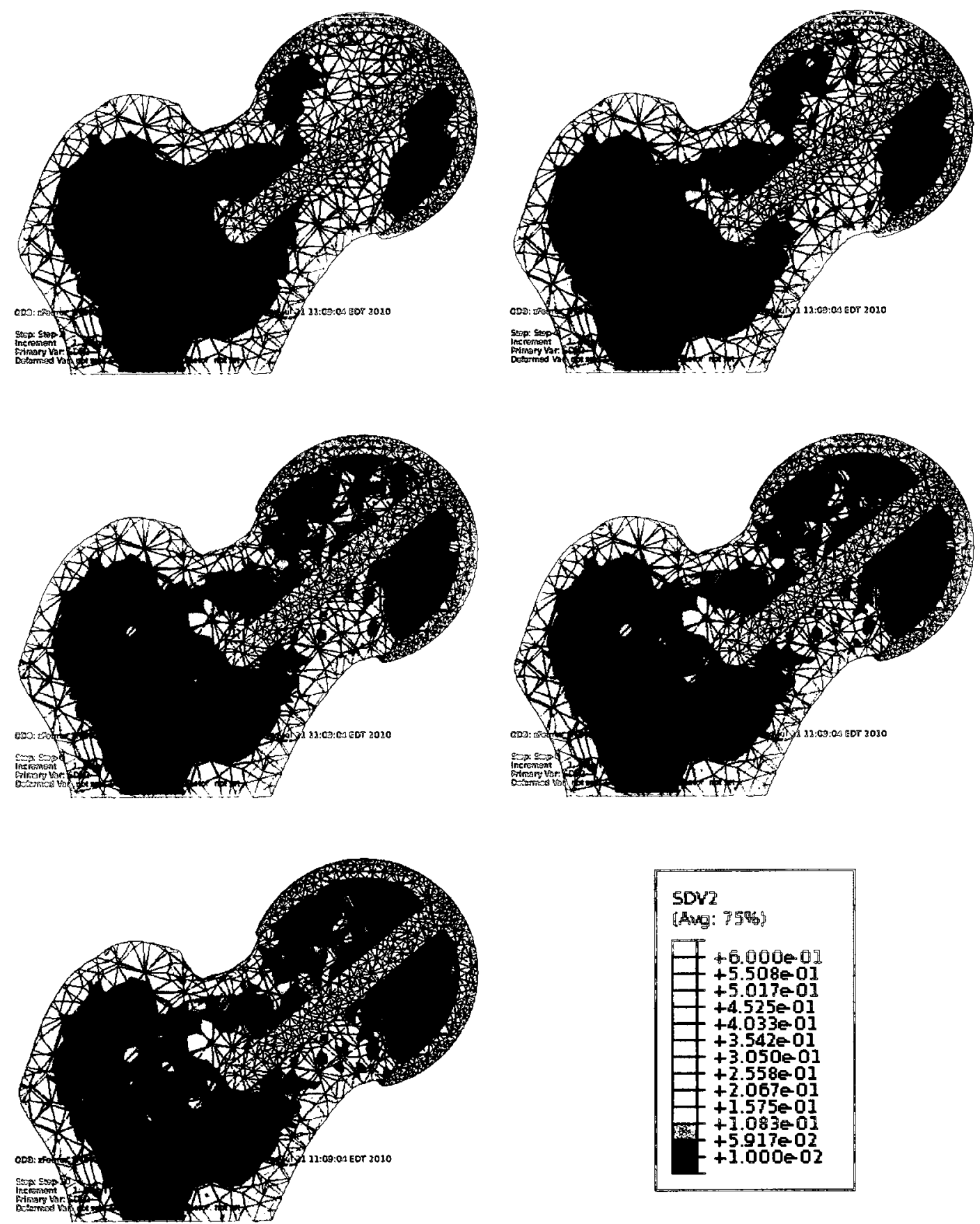

Figure F3: Bone density during analysis of the fixed-friction model. From left to right, top to bottom: step 2, 4, 6, 8, 10. Density values are all in $\mathrm{g} / \mathrm{cc}$. 


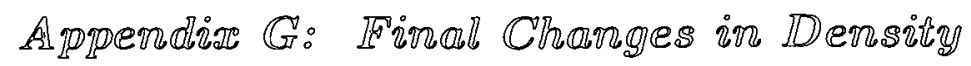

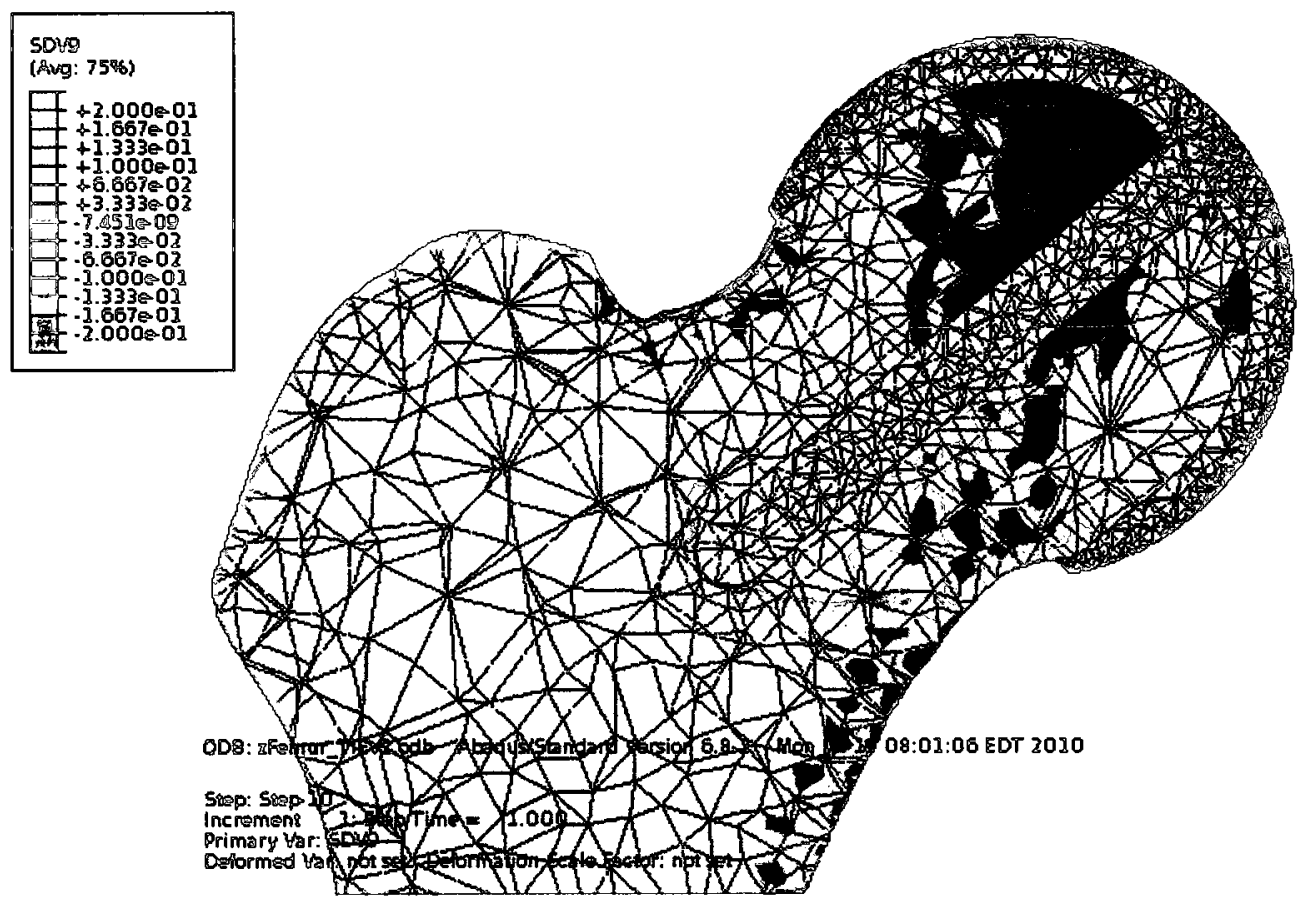

Frigurre $G 1:$ Absolute change in bone density under fully fixed implant. Values are in $\mathrm{g} / \mathrm{cc}$. 


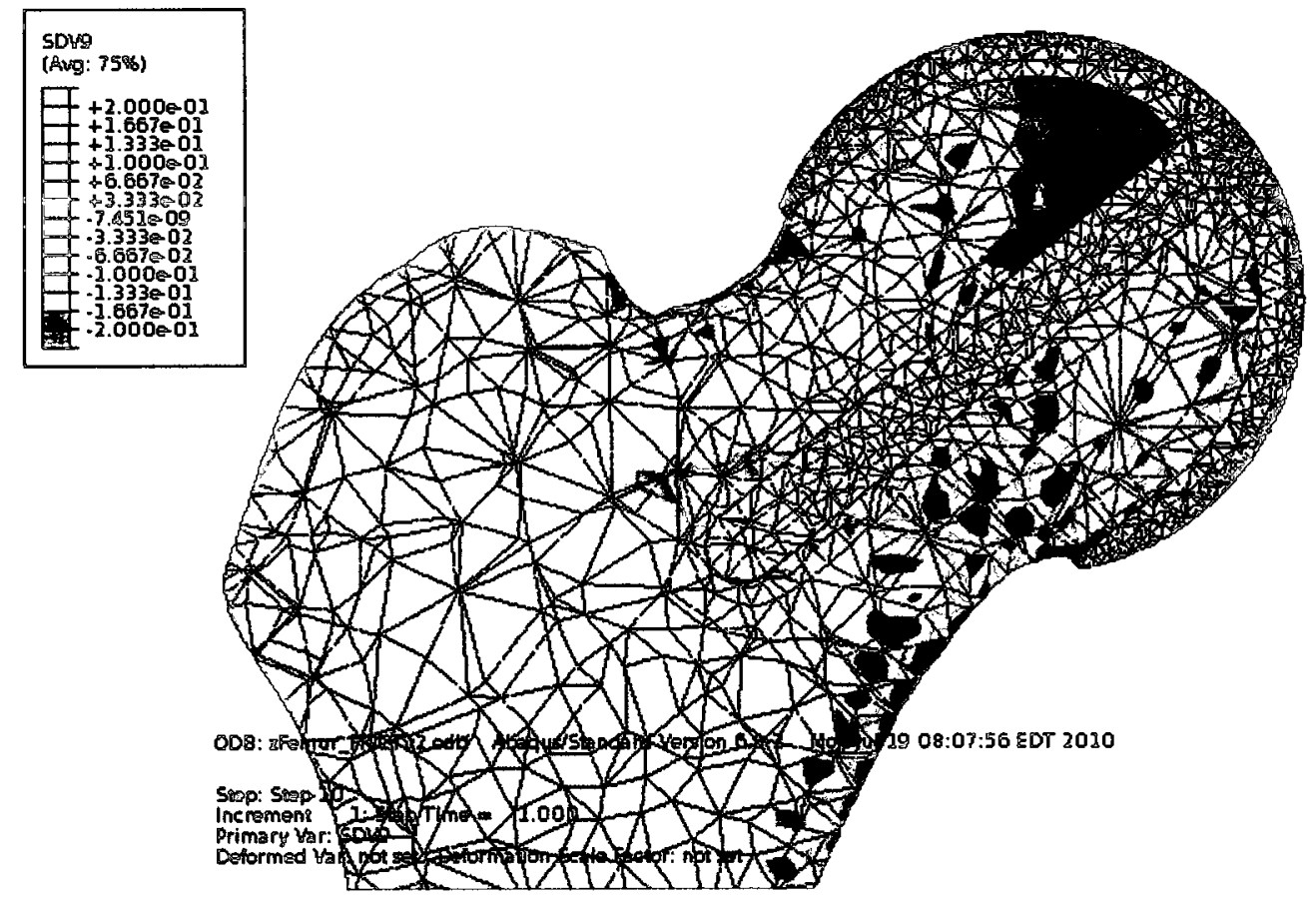

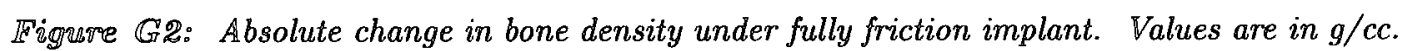




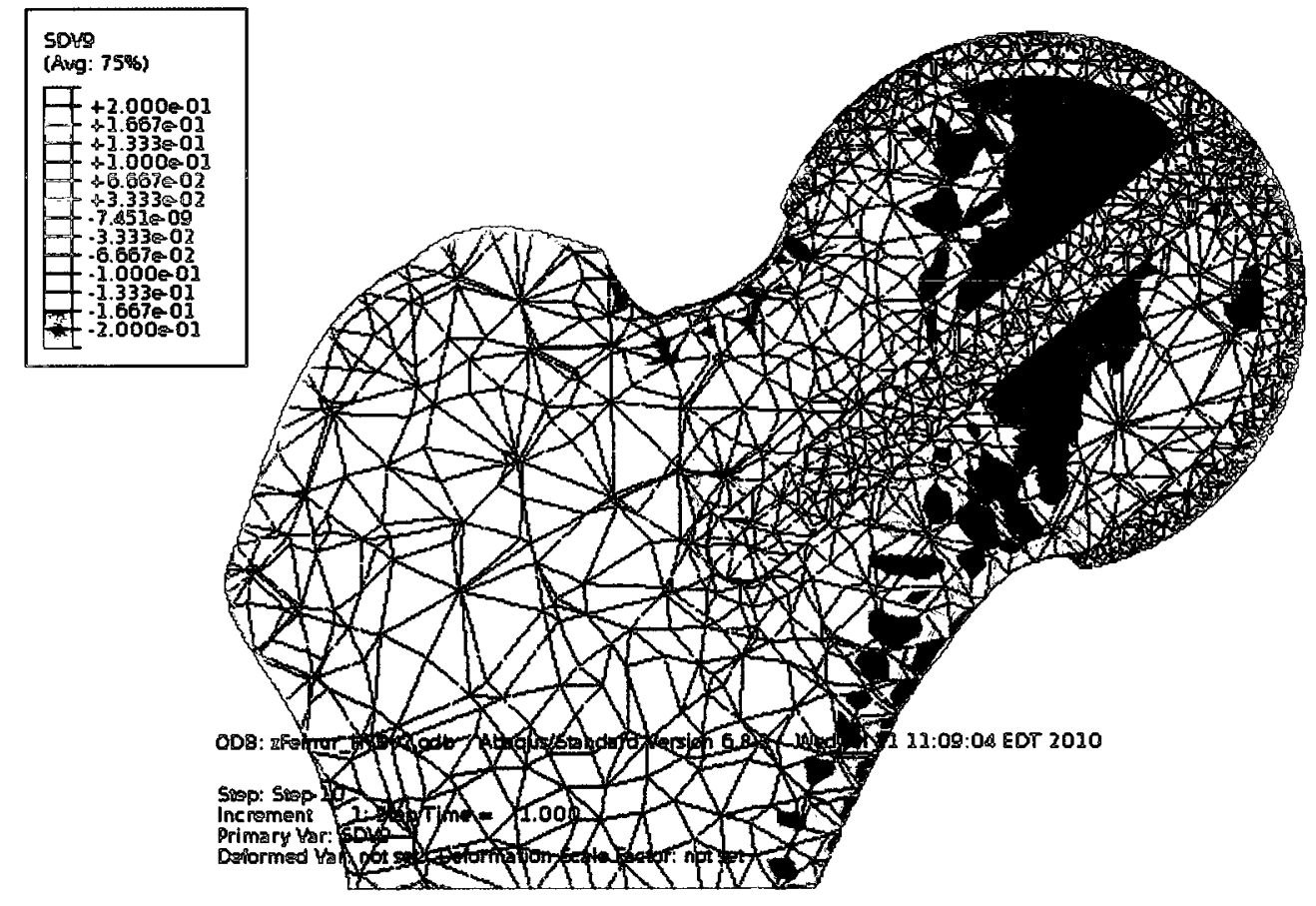

Frigurse GS: Absolute change in bone density under fixed-friction implant. Values are in $\mathrm{g} / \mathrm{cc}$. 


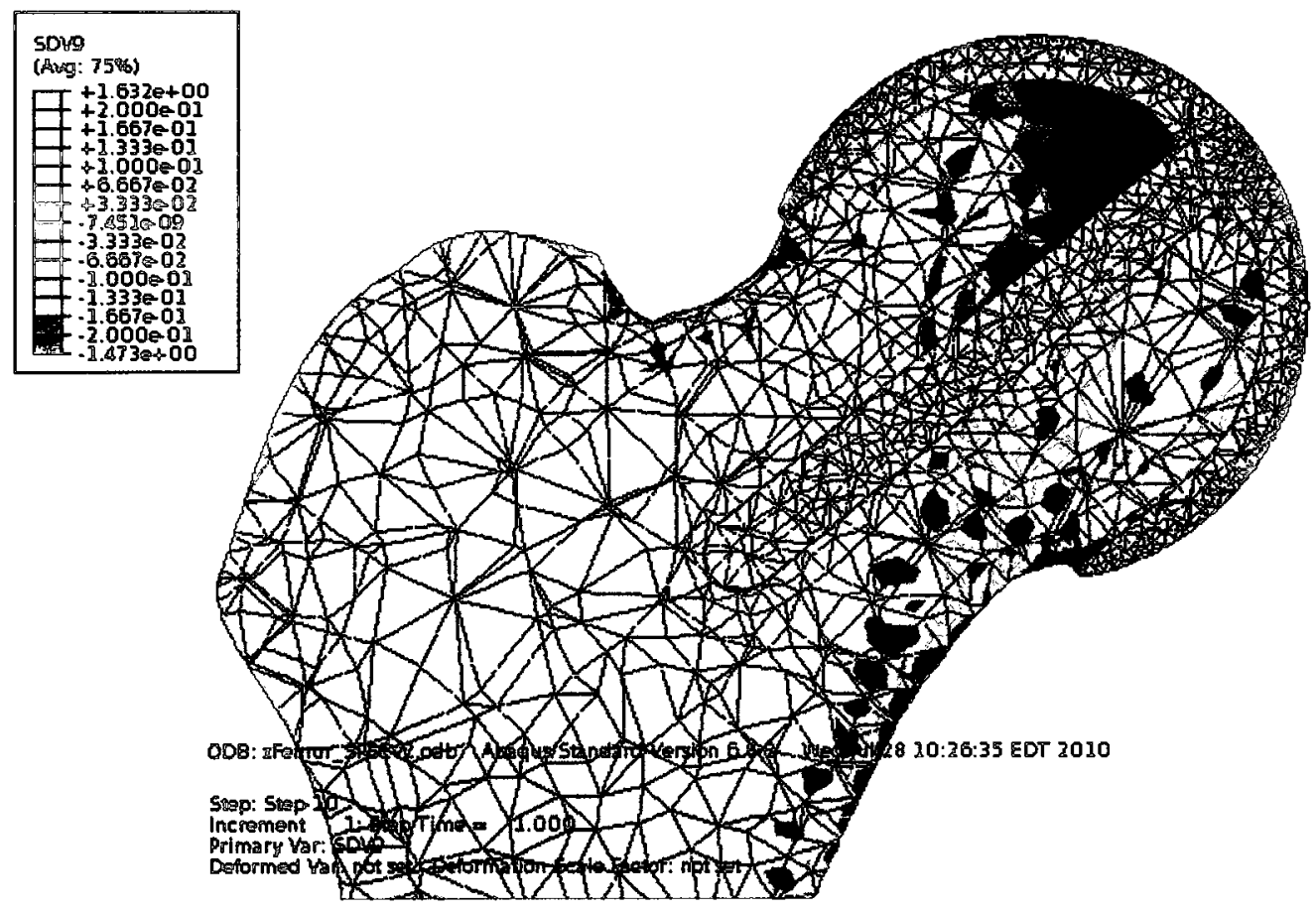

IFigure $G$ G: Final change in bone density under selectively fixed implant. Values are in $\mathrm{g} / \mathrm{cc}$. 


\section{Appendix H: Quilt Plot for Final Density Changes}

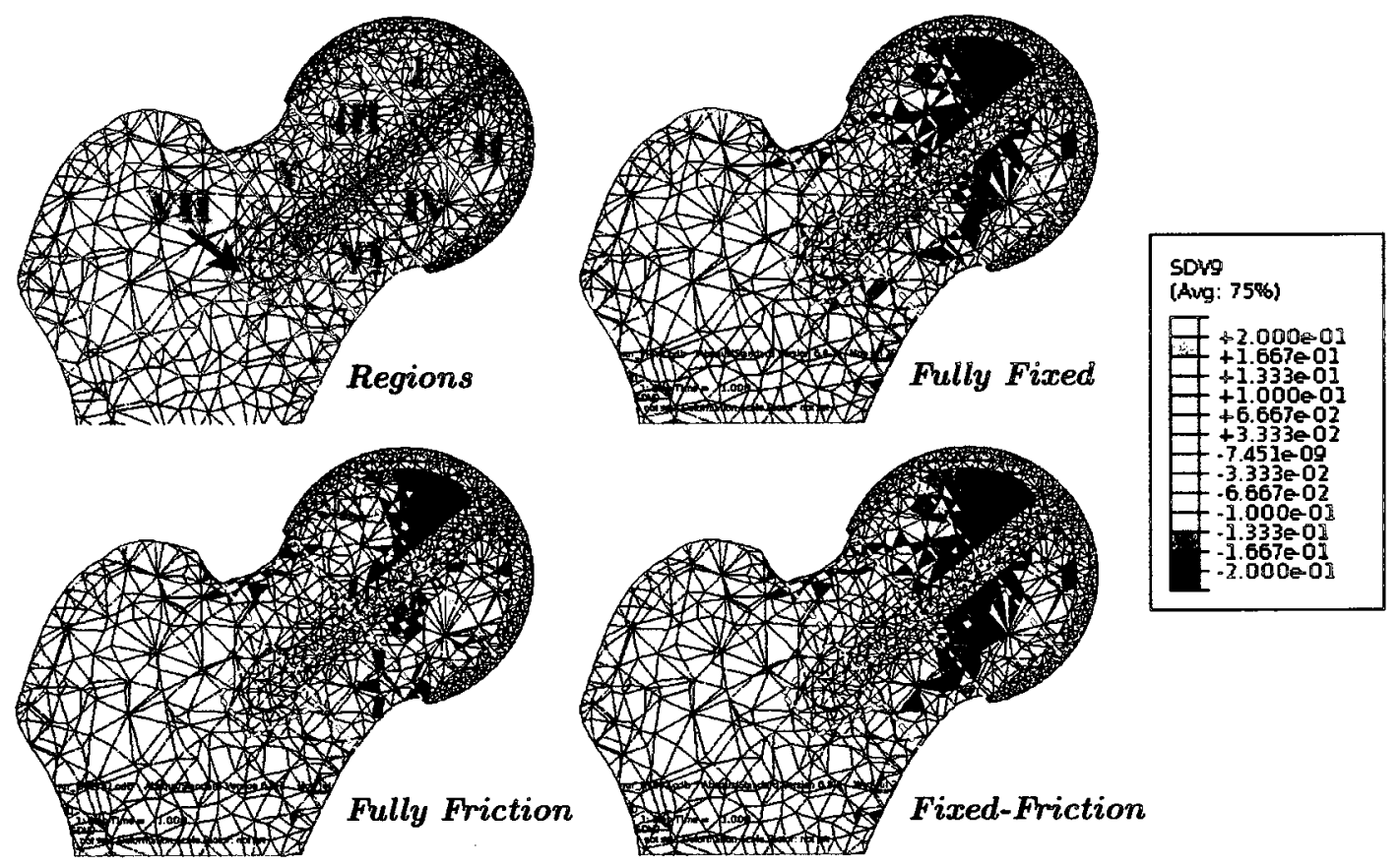

Figure H1: Absolute change in bone density for each implant case output as a quilt. This figure is analogous to Figure 3.5, but plotted as an Abaqus quilt to investigate the impact that output extrapolation has on results interpretation. All relative density values are in $\mathrm{g} / \mathrm{cc}$. 